\title{
Microlocal smoothing effect for Schrödinger equations in Gevrey spaces
}

\author{
By Kunihiko KaJitani and Giovanni Taglialatela
}

(Received Jul. 16, 2001)

\begin{abstract}
The aim of this paper is to investigate the phenomena of microlocal smoothing effect for Schrödinger type equations, in Gevrey spaces. We shall prove that microlocal Gevrey regularity of the solutions of the Cauchy problem for Schrödinger equation, depends on the initial data decay along a backward bicharacteristic.
\end{abstract}

\section{Introduction.}

Let $T>0$. We consider the following Cauchy problem,

$$
\begin{cases}L u=0, & t \in[-T, T], x \in \boldsymbol{R}^{n} \\ u(0, x)=u_{0}(x), & x \in \boldsymbol{R}^{n}\end{cases}
$$

where

$$
L u=\partial_{t} u-\sqrt{-1} \sum_{j, k=1}^{n} \frac{\partial}{\partial x_{j}}\left(a_{j k}(x) \frac{\partial}{\partial x_{k}} u\right)-\sum_{j=1}^{n} b_{j}(t, x) \frac{\partial}{\partial x_{j}} u-b_{0}(t, x) u
$$

$a_{2}(x, \xi)=\sum_{j, k=1}^{n} a_{j k}(x) \xi_{j} \xi_{k}$, is a real elliptic symbol with smooth and bounded coefficients.

We will consider the smoothing effect phenomenon: more the initial data decays at the infinity, more the solution of $(0.1)$ is regular.

Many papers are devoted to the study of this phenomenon: A. Jensen [16] and Hayashi, Nakamitsu and Tsutsumi [14] showed that if $\langle x\rangle^{k} u_{0}(x) \in L^{2}\left(\boldsymbol{R}^{n}\right)$, then the solution $u$ of $[0.1)$ belongs to $H_{l o c}^{k}$ for $t \neq 0$. Hayashi and Saitoh [13] proved that if $e^{c\langle x\rangle^{2}} u_{0} \in L^{2}\left(\boldsymbol{R}^{n}\right), c>0$, then the solution $u$ is analytic in $x$ for $t \neq 0$. Kajitani [19], Kajitani and Wakabayashi [24] proved that if $e^{c\langle x\rangle} u_{0} \in L^{2}\left(\boldsymbol{R}^{n}\right), c>0$, then the solution $u$ is analytic in $x$ for $t \neq 0$. De Bouard, Hayashi and Kato [5] showed that if $u_{0}$ satisfies $\left\|(x \cdot \nabla)^{j} u_{0}\right\| \leq C^{j+1} j !^{d}$ for $j \in N$, then the solution belongs to the Gevrey class $\gamma^{d / 2}$ with respect to $x$ for $t \neq 0$. Kajitani [20], [21] proved that if $e^{c<x\rangle^{\kappa}} u_{0} \in L^{2}\left(\boldsymbol{R}^{n}\right)$, $c>0, \kappa \in] 0,1\left[\right.$, then the solution belongs to the Gevrey class $\gamma^{1 / d}$ with respect to $x$ for $t \neq 0$.

In the case of microlocal smoothing effect, the decay of initial data is required only on a neighborhood of the backward bicharacteristic (see Craig, Kappeler and Strauss [4], Doi [6], [11] for the microlocal $\mathscr{C}^{\infty}$ smoothing effect, and Robbiano and Zuily [27], [28], [29], Robbiano, Zuily and Morimoto [26] for the microlocal analytic smoothing effect). In this paper we will consider the microlocal smoothing effect in Gevrey classes.

2000 Mathematics Subject Classification. Primary 35B65; Secondary 35Q40, 35Q55.

Key Words and Phrases. Microlocal smoothing effect, Schrödinger equation, Hamilton flow. 


\section{Statement of the Theorem.}

We recall that a function $f$ belongs to the Gevrey class $\gamma^{s}(\Omega), \Omega$ open subset of $\boldsymbol{R}^{n}$ if $f \in \mathscr{C}^{\infty}(\Omega ; \boldsymbol{C})$ and for any compact $K$ there exist positive constants $C=C(K)$ and $\rho=\rho(K)$ such that

$$
\sup _{x \in K}\left|D^{\alpha} f(x)\right| \leq C \rho^{-|\alpha|}|\alpha| !^{s}
$$

for all $\alpha \in \boldsymbol{N}^{n}$. One says that a point $\left(y_{0}, \eta_{0}\right) \in \boldsymbol{R}^{n} \times \boldsymbol{R}^{n} \backslash\{0\}$ does not belong to the Gevrey wave front set of order $d$ of a distribution $u$ if there exists a function $\chi \in \mathscr{C}_{0}^{\infty}\left(\boldsymbol{R}^{n}\right)$, equal to 1 in a neighborhood of 0 , such that if one sets $\chi_{y_{0}}(x)=$ $\chi\left(x-y_{0}\right)$ and $\chi_{\eta_{0}}(\xi)=\chi\left((\xi /|\xi|)-\left(\eta_{0} /\left|\eta_{0}\right|\right)\right)$, then there exist positive constants $C$ and $\rho$ such that

$$
\left|D_{x}^{\alpha}\left(\chi_{\eta_{0}}(D)\left(\chi_{y_{0}}(x) u\right)\right)\right| \leq C \rho^{-|\alpha|}|\alpha| !^{d},
$$

for all $\alpha \in N^{n}$. We will note $\mathrm{WF}_{d} u$ the wave front set of order $d$ of $u$.

Assumption I.

(1) $a_{j k} \in \gamma^{d}\left(\boldsymbol{R}^{n}\right)$, for some $d \geq 1$;

(2) there exists positive constants $C_{1}$ and $C_{2}$ such that

$$
C_{1}|\xi|^{2} \leq\left|a_{2}(x, \xi)\right| \leq C_{2}|\xi|^{2}
$$

for all $\xi \in \boldsymbol{R}^{n}$;

(3) there exist positive constants $\delta, \rho$ and $C$ such that

$$
\left|D_{x}^{\alpha} a_{j k}(x)\right| \leq C_{\rho} \rho^{-|\alpha|}|\alpha| !^{d}\langle x\rangle^{-|\alpha|-\delta},
$$

for all $x \in \boldsymbol{R}^{n}, \alpha \in \boldsymbol{N}^{n} \backslash\{0\}$, and $j, k=1, \ldots, n$;

(4) there exists a function $\theta(x, \xi) \in \mathscr{C}^{\infty}\left(\boldsymbol{R}^{n} \times \boldsymbol{R}^{n}\right)$ such that

(a) there exist positive constants $\rho$ and $C$ such that

$$
\left|D_{\xi}^{\alpha} D_{x}^{\beta} \theta(x, \xi)\right| \leq C_{\rho} \rho^{-|\alpha+\beta|}|\alpha| !|\beta|^{d}|\xi|^{1-|\alpha|}\langle x\rangle^{1-|\beta|},
$$

for all $x, \xi \in \boldsymbol{R}^{n}, \alpha, \beta \in \boldsymbol{N}^{n}$;

(b) $H_{a_{2}} \theta(x, \xi) \geq C|\xi|^{2}$, for some positive constant $C$, where $H_{a_{2}}$ is the Hamiltonian flow associated to $a_{2}$ :

$$
H_{a_{2}}=\sum_{j=1}^{n}\left(\frac{\partial a_{2}}{\partial \xi_{j}} \frac{\partial}{\partial x_{j}}-\frac{\partial a_{2}}{\partial x_{j}} \frac{\partial}{\partial \xi_{j}}\right) .
$$

EXAMPLE 1.1. If $a_{2}(x, \xi)=|\xi|^{2}$, we can take $\theta(x, \xi)=x \cdot \xi$.

More generally, if $a_{2}(x, \xi)=\sum_{j, k=1}^{n} a_{j k} \xi_{j} \xi_{k}$ has constant coefficients, we can take

$$
\theta(x, \xi)=x \cdot a_{2 \xi}(x, \xi)=\sum_{j=1}^{n} x_{j} \partial_{\xi_{j}} a_{2}(x, \xi)=2 \sum_{j, k=1}^{n} a_{j k} x_{j} \xi_{k} .
$$

Indeed we have 


$$
H_{a_{2}} \theta(x, \xi)=4 \sum_{j=1}^{n}\left[\sum_{k=1}^{n} a_{j k} \xi_{k}\right]^{2}=4\left|\operatorname{grad}_{\xi} a_{2}(\xi)\right|^{2}
$$

Now, $\operatorname{grad}_{\xi} a_{2}(\xi)$ cannot vanishes for $\xi \neq 0$, or, by Euler formula, $a_{2}(\xi)$ must vanish too. So, if $C$ is the minimum of $\left|\operatorname{grad}_{\xi} a_{2}(\xi)\right|$ for $|\xi|=1$, then $H_{a_{2}} \theta(x, \xi) \geq C|\xi|^{2}$.

EXAMPLE 1.2. If $a_{2}(x, \xi)=\sum_{j=1}^{n} a_{j}\left(x_{j}\right) \xi_{j}^{2}$, with $C_{1} \leq a_{j}\left(x_{j}\right) \leq C_{2}$, we can choose $\theta(x, \xi)=\sum_{j=1}^{n} x_{j} \sqrt{a_{j}\left(x_{j}\right)} \xi_{j}$. Indeed, a direct computation shows that

$$
\begin{aligned}
H_{a_{2}} \theta(x, \xi) & =\sum_{j=1}^{n} 2 a_{j}\left(x_{j}\right) \xi_{j}\left[\sqrt{a_{j}\left(x_{j}\right)} \xi_{j}+x_{j} \frac{a_{j}^{\prime}\left(x_{j}\right)}{2 \sqrt{a_{j}\left(x_{j}\right)}} \xi_{j}\right]-\sum_{j=1}^{n} a_{j}^{\prime}\left(x_{j}\right) \xi_{j}^{2} x_{j} \sqrt{a_{j}\left(x_{j}\right)} \\
& =\sum_{j=1}^{n} 2\left[a_{j}\left(x_{j}\right)\right]^{3 / 2} \xi_{j}^{2} \geq 2 C_{1}^{3 / 2}|\xi|^{2} .
\end{aligned}
$$

Assumption II.

(1) $b_{j} \in \mathscr{C}\left([0, T] ; \gamma^{d}\left(\boldsymbol{R}^{n}\right)\right)$, for $j=0,1, \ldots, n$ and there exist positive constants $\rho$ and $C$ such that

$$
\left|D_{x}^{\alpha} b_{j}(t, x)\right| \leq C_{\rho} \rho^{-|\alpha|}|\alpha| !^{d}\langle x\rangle^{-|\alpha|},
$$

for all $x \in \boldsymbol{R}^{n}, \alpha \in \boldsymbol{N}^{n} \backslash\{0\}$, for all $j=0,1, \ldots, n$;

(2) $\operatorname{Im} b_{j}(t, x)=0$, for $j=1, \ldots, n$.

For $\left(y_{0}, \eta_{0}\right) \in \boldsymbol{R}^{n} \times \boldsymbol{R}^{n} \backslash\{0\}$, we consider the bicharacteristic of $a_{2}(x, \xi)$ passing through $\left(y_{0}, \eta_{0}\right)$ :

$$
\left\{\begin{array}{l}
\dot{X}(s)=\frac{\partial a_{2}}{\partial \xi}(X(s), \Xi(s)) \\
\dot{\Xi}(s)=-\frac{\partial a_{2}}{\partial x}(X(s), \Xi(s))
\end{array}(X(0), \Xi(0))=\left(y_{0}, \eta_{0}\right)\right.
$$

where $\dot{X}=\frac{d}{d t} X$ and $\dot{\Xi}=\frac{d}{d t} \Xi$.

Since $a_{2}(x, \xi)$ is elliptic, the solution of (1.2) exists for all $s \in \boldsymbol{R}$. Moreover, the existence of the function $\theta(x, \xi)$ implies that (see Remark 4.6 below)

$$
\lim _{s \rightarrow \pm \infty}\left|X\left(s ; y_{0}, \eta_{0}\right)\right|=+\infty .
$$

For $\left(y_{0}, \eta_{0}\right) \in \boldsymbol{R}^{n} \times \boldsymbol{R}^{n} \backslash\{0\}$, let $V_{\varepsilon}\left(\eta_{0}\right)=\left\{\xi \in \boldsymbol{R}^{n} \backslash\{0\}||(\xi /|\xi|)-\left(\eta_{0} /\left|\eta_{0}\right|\right) \mid<\varepsilon\right\}$ and

$$
\Gamma_{\varepsilon}^{ \pm}\left(y_{0}, \eta_{0}\right)=\bigcup_{s \geq 0}\left\{x \in \boldsymbol{R}^{n}|| x-X\left(-s ; y_{0}, \eta_{0}\right) \mid \leq \varepsilon(1+s)\right\}
$$

For $\varepsilon \in \boldsymbol{R}$ denote

$$
\varphi_{\varepsilon}^{ \pm}(x, \xi)=x \cdot \xi-i \varepsilon \frac{\theta(x, \xi)}{\langle x\rangle^{1-\sigma}\langle\xi\rangle^{1-\delta}} \chi^{ \pm}(x, \xi),
$$

where $\sigma>0, \delta \geq 0, \sigma+\delta=\kappa, \kappa<1$, and $\chi^{ \pm}(x, \xi) \in \gamma^{d}\left(\boldsymbol{R}^{n} \times \boldsymbol{R}^{n}\right)$ is such that $\chi^{ \pm}(x, \xi)=1$ 
if $(x, \xi) \in \Gamma_{\varepsilon}^{ \pm}\left(y_{0}, \eta_{0}\right) \times V_{\varepsilon} \cap\{\xi|| \xi \mid \geq 1\}$ and $\chi^{ \pm}(x, \xi)=0$ if $(x, \xi) \notin \Gamma_{2 \varepsilon}^{ \pm}\left(y_{0}, \eta_{0}\right) \times V_{2 \varepsilon}$ and define

$$
I_{\varphi_{\varepsilon}^{ \pm}}(x, D) u(x)=\int_{\boldsymbol{R}^{n}} e^{i \varphi_{\varepsilon}^{ \pm}(x, \xi)} \hat{u}(\xi) \bar{d} \xi,
$$

where $\hat{u}(\xi)$ stands for a Fourier transform of $u$ and $\bar{d} \xi=(2 \pi)^{-n} d \xi$.

Now we can state our result.

Theorem. Assume that Assumptions I and II are satisfied for $d \kappa \leq 1, d \geq 1$ and $\kappa<1$. If $u_{0} \in L^{2}\left(\boldsymbol{R}^{n}\right)$ and $I_{\varphi_{\varepsilon}^{ \pm}}(x, D) u_{0} \in L^{2}\left(\boldsymbol{R}^{n}\right)$, for $|\varepsilon| \leq \varepsilon_{0}$, then there exists a solution $u$ of $(0.1)$ such that $\left(y_{0}, \eta_{0}\right) \notin \mathrm{WF}_{1 / \kappa} u(t, \cdot)$, for all $t>0$.

EXAMPLE 1.3. If $e^{\varepsilon_{0}\langle x\rangle^{\kappa}} u_{0} \in L^{2}\left(\Gamma_{\varepsilon_{0}}^{ \pm}\left(y_{0}, \eta_{0}\right)\right)$, or $e^{\varepsilon_{0}\langle x\rangle^{\sigma}\langle D\rangle^{\delta}} u_{0} \in L^{2}\left(\Gamma_{\varepsilon_{0}}^{ \pm}\left(y_{0}, \eta_{0}\right)\right)$, with $\sigma>0, \delta \geq 0, \sigma+\delta=\kappa$, then $I_{\varphi_{\varepsilon_{0}}^{ \pm}} u_{0} \in L^{2}\left(\Gamma_{\varepsilon_{0}}^{ \pm}\left(y_{0}, \eta_{0}\right)\right)$.

Indeed, one can easily show that if $\varphi_{\varepsilon}^{(j)}(x, \xi)=x \cdot \xi-i \varepsilon \tilde{\varphi}^{(j)}(x, \xi)$, for $j=1,2$, and there exists $C>0$ such that $\left|\tilde{\varphi}^{(1)}(x, \xi)\right| \leq C \tilde{\varphi}^{(2)}(x, \xi)$, then if $I_{\varphi_{\varepsilon_{0}}^{(2)}} u_{0} \in L^{2}\left(\boldsymbol{R}^{n}\right)$ for some $\varepsilon_{0}>0$, then there exists $\varepsilon_{0}^{\prime}>0$ such that $I_{\varphi_{\varepsilon}^{(1)}} u_{0} \in L^{2}\left(\boldsymbol{R}^{n}\right)$, for all $|\varepsilon|<\varepsilon_{0}^{\prime}$. So, it's enough to remark that

$$
\left|\frac{\theta(x, \xi)}{\langle x\rangle^{1-\sigma}\langle\xi\rangle^{1-\delta}}\right| \leq C\langle x\rangle^{\sigma}\langle\xi\rangle^{\delta} .
$$

REMARK 1.4. By similar argument, we can see that we can assume, without loss of generality, that $\theta\left(y_{0}, \eta_{0}\right)=0$.

REMARK 1.5. We will assume in the following that $a_{2}(x, \xi)$ is positive, the case when $a_{2}(x, \xi)$ is negative is treated by changing time orientation.

\section{Preliminaries.}

In this section we recall some results that we will use in the proof of the Theorem. For the proofs see [19], [20], [21] and [24].

\subsection{Basic inequalities.}

The following inequalities are used in this paper

Lemma 2.1 ([12, Lemma 12.1]). There exists $c>0$ such that for any $s>0$

$$
\inf _{N \in N} \frac{N !}{S^{N}} \leq c e^{-s / 2}
$$

Lemma 2.2 ([20, Lemma 5.1]). For any $r, s \in \boldsymbol{N}, \alpha \in \boldsymbol{N}^{n}, q>1$ and $s \geq 1$, we have

$$
\sum_{\alpha^{\prime}+\alpha^{\prime \prime}=\alpha}\left(\begin{array}{c}
\alpha \\
\alpha^{\prime}
\end{array}\right)\left(\left|\alpha^{\prime}\right|+r\right) !^{d}\left(\left|\alpha^{\prime \prime}\right|+s\right) !^{d} q^{-\left|\alpha^{\prime}\right|} \leq\left(\begin{array}{c}
r+s \\
r
\end{array}\right)^{-1}(|\alpha|+r+s) !^{d} \frac{q}{q-1} .
$$

Lemma 2.3. Let $r, s, t \in N$, with $t \leq r, \alpha \in N^{n}, d \geq 1$ and $q>2^{d}$, then

$$
\sum_{\substack{\alpha^{\prime}+\alpha^{\prime \prime}=\alpha \\
\left|\alpha^{\prime}\right| \geq k}}\left(\begin{array}{c}
\alpha \\
\alpha^{\prime}
\end{array}\right)\left(\left|\alpha^{\prime}\right|+r\right) !^{d}\left(\left|\alpha^{\prime \prime}\right|+s\right) !^{d} q^{-\left|\alpha^{\prime}\right|} \leq \frac{2^{d r} t !^{d}}{\left(\begin{array}{c}
r+s-t \\
r-t
\end{array}\right)^{d}}(|\alpha|+r+s-t) !^{d} \frac{\left(2^{d} / q\right)^{k}}{1-\left(2^{d} / q\right)} \text {. }
$$


The proof is similar to the proof of the previous Lemma, and is given in the appendix.

\subsection{Sobolev spaces with exponential weights.}

In the following we note $\mathscr{F} u$ or $\hat{u}$ the Fourier transform of $u$, and with $\mathscr{F}^{-1}$ its inverse.

Let $\kappa \in] 0,1[$. For $\rho \geq 0$ and $\delta \in \boldsymbol{R}$, let

$$
H_{\rho, \delta}^{\kappa}=\left\{u \in L_{\mathrm{loc}}^{2}\left(\boldsymbol{R}^{n}\right) \mid e^{\rho\langle D\rangle^{\kappa}} u \in L^{2}\left(\boldsymbol{R}^{n}\right), e^{\delta\langle x\rangle^{\kappa}} e^{\rho\langle D\rangle^{\kappa}} u \in L^{2}\left(\boldsymbol{R}^{n}\right)\right\},
$$

and for $\rho<0 H_{\rho, \delta}^{\kappa}$ is the dual space of $H_{-\rho,-\delta}^{\kappa}$. Here $e^{\rho\langle D\rangle^{\kappa}} u=\int_{\boldsymbol{R}^{n}} e^{i x \xi} e^{\rho\langle\xi\rangle^{\kappa}} \hat{u}(\xi) \bar{d} \xi$.

Proposition 2.4. If $u \in H_{\rho, \delta}^{\kappa}$, with $\rho>0$, then for any $0<\rho^{\prime}<\rho$ there exists a constant $C_{\rho, \rho^{\prime}}$ such that

$$
\left|\partial_{x}^{\alpha} u(x)\right| \leq C_{\rho, \rho^{\prime}} \rho^{-|\alpha|}|\alpha| !^{1 / \kappa} e^{\delta\langle x\rangle^{\kappa}}\|u\|_{H_{\rho, \delta}^{\kappa},}
$$

for any $x \in \boldsymbol{R}^{n}, \alpha \in \boldsymbol{N}^{n}$.

In particular, $u$ is a Gevrey function.

\subsection{Symbols.}

Let $m, \varphi, \psi$ be weight functions and $g=\varphi^{-2} d x^{2}+\psi^{-2} d \xi^{2}$ a Riemann metric. We will consider essentially $g_{0}=d x^{2}+d \xi^{2}$ and $g_{1}=\langle x\rangle^{-2} d x^{2}+\langle\xi\rangle^{-2} d \xi^{2}$. Let $S(m, g)$ the set of symbols $a(x, \xi) \in \mathscr{C}^{\infty}\left(\boldsymbol{R}^{2 n}\right)$ satisfying

$$
\left|a_{(\beta)}^{(\alpha)}(x, \xi)\right| \leq C_{\alpha, \beta} m(x, \xi) \varphi^{-|\alpha|} \psi^{-|\beta|}
$$

for $x, \xi \in \boldsymbol{R}^{n}, \alpha, \beta \in \boldsymbol{N}^{n}$, where $a_{(\beta)}^{(\alpha)}=\partial_{\xi}^{\alpha} D_{x}^{\beta} a$, and $\gamma^{d, s} S(m, g)$ the set of the functions $a(x, \xi) \in \mathscr{C}^{\infty}\left(\boldsymbol{R}^{2 n}\right)$ satisfying the following condition: there exist $\rho_{a}>0$ and $C_{a}>0$ such that

$$
\left|a_{(\beta)}^{(\alpha)}(x, \xi)\right| \leq C_{a} \rho_{a}^{-|\alpha+\beta|}|\alpha| !^{s}|\beta| !^{d} m(x, \xi) \varphi^{-|\alpha|} \psi^{-|\beta|}
$$

for $x, \xi \in \boldsymbol{R}^{n}, \alpha, \beta \in \boldsymbol{N}^{n}$.

If $d=s$, we write, for short, $\gamma^{d} S(m, g)=\gamma^{d, d} S(m, g)$. Without introducing new notations, we will write also $a \in \gamma^{d, s} S(m, g)$, for symbols $a(x, \xi) \in \mathscr{C}^{\infty}\left(\boldsymbol{R}^{n} \times \boldsymbol{R}^{n} \backslash\{0\}\right)$, satisfying (2.2) in $\boldsymbol{R}^{n} \times \boldsymbol{R}^{n} \backslash\{0\}$.

EXAMPLE 2.5. The symbol $a_{2}(x, \xi)=\sum_{j, k=1}^{n} a_{j k}(x) \xi_{j} \xi_{k}$ belongs to $\gamma^{d, 1} S\left(\langle\xi\rangle^{2}, g_{1}\right)$, $\theta(x, \xi)$ belongs to $\gamma^{d, 1} S\left(\langle x\rangle|\xi|, g_{1}\right)$, and $b(t, x, \xi)=\sum_{j=1}^{n} b_{j}(t, x) \xi_{j}$ belongs to $\mathscr{C}^{0}([-T, T]$; $\left.\gamma^{d, 1} S\left(\langle\xi\rangle, g_{1}\right)\right)$.

For $\kappa \in] 0,1\left[\right.$, let $\mathscr{A}_{\rho, \delta}^{\kappa}=\gamma^{1 / \kappa} S\left(e^{\delta\langle x\rangle^{\kappa}+\rho\langle\xi\rangle^{\kappa}}, g_{0}\right)$, with $g_{0}=d x^{2}+d \xi^{2}$. For $a_{i} \in$ $\mathscr{A}_{\rho_{i}, \delta_{i}}^{\kappa}, i=1,2$, the product of $a_{1}$ and $a_{2}$ is defined as follows

$$
\begin{aligned}
\left(a_{1} \circ a_{2}\right)(x, \xi) & =\text { os- } \iint_{\boldsymbol{R}^{2 n}} e^{-i y \eta} a_{1}(x, \xi+\eta) a_{2}(x+y, \xi) d y \bar{d} \eta \\
& =\lim _{\varepsilon \rightarrow 0} \iint_{\boldsymbol{R}^{2 n}} e^{-i y \eta-\varepsilon\left(|y|^{2}+|\eta|^{2}\right)} a_{1}(x, \xi+\eta) a_{2}(x+y, \xi) d y \bar{d} \eta
\end{aligned}
$$

where $\bar{d} \eta=(2 \pi)^{-1} d \eta$. 
PROPOSITION 2.6.

(1) Let $\kappa \in] 0,1\left[\right.$, and $a_{i} \in \mathscr{A}_{\rho_{i}, \delta_{i}}^{\kappa}, i=1,2$. There exists $\varepsilon^{*}>0$ such that if $\left|\rho_{1}\right|$, $\left|\delta_{2}\right| \leq \varepsilon^{*}$, then $a_{1} \circ a_{2}$ belongs to $\mathscr{A}_{\rho_{1}+\rho_{2}, \delta_{1}+\delta_{2}}$.

(2) Let $\kappa \in] 0,1\left[\right.$, and $a_{i} \in \mathscr{A}_{\rho_{i}, \delta_{i}}^{\kappa}, i=1,2,3$. Then if $\left|\rho_{1}\right|,\left|\rho_{2}\right|,\left|\delta_{2}\right|,\left|\delta_{3}\right| \leq \varepsilon^{*} / 2$ we have $\left(a_{1} \circ a_{2}\right) \circ a_{3}=a_{1} \circ\left(a_{2} \circ a_{3}\right)$.

Proposition 2.7. Let $d_{i}, s_{i} \geq 1$ and $a_{i} \in \gamma^{d_{i}, s_{i}} S\left(\langle x\rangle^{m_{i}}\langle\xi\rangle^{\ell_{i}}, g_{1}\right), \quad i=1,2$. Then $a_{1} \circ a_{2}$ belongs to $\gamma^{d, s} S\left(\langle x\rangle^{m_{1}+m_{2}}\langle\xi\rangle^{\ell_{1}+\ell_{2}}, g_{1}\right)$, where $d=\min \left(d_{1}, d_{2}\right), \quad s=\min \left(s_{1}, s_{2}\right)$. Moreover we can decompose

$$
\left(a_{1} \circ a_{2}\right)(x, \xi)=p(x, \xi)+r(x, \xi),
$$

where

- $p(x, \xi) \in \gamma^{d, s} S\left(\langle x\rangle^{m_{1}+m_{2}}\langle\xi\rangle^{\ell_{1}+\ell_{2}}, g_{1}\right)$ satisfies for $C>0$

$$
\begin{aligned}
& p(x, \xi)-\sum_{|\gamma|<N} \frac{1}{\gamma !} a_{1}^{(\gamma)}(x, \xi) a_{2(\gamma)}(x, \xi) \\
& \quad \in \gamma^{d, s} S\left(C^{N+1} N !^{s_{1}+d_{2}-1}\langle x\rangle^{m_{1}+m_{2}-N}\langle\xi\rangle^{\ell_{1}+\ell_{2}-N}, g_{1}\right)
\end{aligned}
$$

for any $N \in N$,

- $r(x, \xi) \in \mathscr{A}_{-\varepsilon^{*},-\varepsilon^{*}}^{1 / d}$.

LeMma 2.8. Let $f(x, \xi), \varphi_{i}(x, \xi), \psi_{i}(x, \xi) \in \mathscr{C}^{\infty}\left(\boldsymbol{R}^{n} \times \boldsymbol{R}^{n} \backslash\{0\}\right), i=1, \ldots, n$ such that

$$
\begin{aligned}
& \left|\partial_{\xi}^{\alpha} \partial_{x}^{\beta} f(x, \xi)\right| \leq C|\alpha| !^{s}|\beta| !^{d} \rho_{0}^{-|\alpha+\beta|} m(x, \xi)\langle x\rangle^{-|\beta|}|\xi|^{-|\alpha|} \\
& \left|\left(\varphi_{i}\right)_{(\beta)}^{(\alpha)}(x, \xi)\right| \leq C|\alpha| !^{s}|\beta| !^{d} \rho_{0}^{-|\alpha+\beta|} m_{\varphi}(x, \xi) \eta_{1}(x, \xi)^{-|\alpha|} \eta_{2}(x, \xi)^{-|\beta|} \\
& \left|\left(\psi_{i}\right)_{(\beta)}^{(\alpha)}(x, \xi)\right| \leq C|\alpha| !^{s}|\beta| !^{d} \rho_{0}^{-|\alpha+\beta|} m_{\psi}(x, \xi) \eta_{1}(x, \xi)^{-|\alpha|} \eta_{2}(x, \xi)^{-|\beta|}
\end{aligned}
$$

for $|\alpha+\beta| \leq M$, for some weight functions $m, m_{\varphi}, m_{\psi}, \eta_{1}$ and $\eta_{2}$ and constants $C$ and $\rho_{0}$. Then

$$
\begin{aligned}
\left|f(\varphi(x, \xi), \psi(x, \xi))_{(\beta)}^{(\alpha)}\right| \leq & |\alpha+\beta| !^{d} \rho^{-|\alpha+\beta|}\left[1+C\left(\frac{m_{\varphi}(x, \xi)}{\langle\varphi(x, \xi)\rangle}+\frac{m_{\psi}(x, \xi)}{|\psi(x, \xi)|}\right)\right]^{|\alpha+\beta|} \\
& \times m(\varphi(x, \xi), \psi(x, \xi)) \eta_{1}(x, \xi)^{-|\alpha|} \eta_{2}(x, \xi)^{-|\beta|}
\end{aligned}
$$

for some positive constants $\rho$ and $C$ depending only on $s, d, \rho_{0}$ and $n$.

The proof is similar to those of Lemma A.1 in [18] and Lemma 5.3 in [23], and is given in the Appendix.

In particular, we can see that if $a \in \gamma^{d, s} S(m, g)$ and $|a(x, \xi)| \geq C_{a}^{-1} m(x, \xi)^{-1}$, then $a^{r} \in \gamma^{d, s} S\left(m^{r}, g\right)$, for all $r \in \boldsymbol{R}$.

For $a(x, \xi) \in \gamma^{d} S\left(m, g_{1}\right)$, an almost analytic extension of $a$ is defined by

$$
a(z, \zeta)=a(x+i y, \xi+i \eta)=\sum_{\alpha, \beta} \frac{(i \eta)^{\alpha}}{\alpha !} \frac{(-y)^{\beta}}{\beta !} \chi\left(b_{\beta} \frac{|y|}{\langle x\rangle}\right) \chi\left(b_{\alpha} \frac{|\eta|}{\langle\xi\rangle}\right) a_{(\beta)}^{(\alpha)}(x, \xi),
$$

where $b_{\alpha}=4^{n} B|\alpha| !^{(d-1) /|\alpha|} / \rho^{a}$, if $d>1$. 
Proposition 2.9. Let $a(x, \xi) \in \gamma^{d} S\left(m, g_{1}\right)$, and $d>1$, then the almost analytic extension of a satisfies the following properties

$$
\begin{aligned}
& \left|\partial_{\xi}^{\alpha} D_{x}^{\beta} \partial_{\eta}^{\gamma} D_{y}^{\delta} a(x+i y, \xi+i \eta)\right| \\
& \leq C m(x, \xi)\left(C \rho_{a}\right)^{-|\alpha+\beta+\gamma+\delta|}\langle\xi\rangle^{-|\alpha|}\langle x\rangle^{-|\beta|}\langle\eta\rangle^{-|\gamma|}\langle y\rangle^{-|\delta|}|\alpha+\beta+\gamma+\delta|^{d} \\
& \left|\left(\partial_{x_{j}}+i \partial_{y_{j}}\right) \partial_{\xi}^{\alpha} D_{x}^{\beta} \partial_{\eta}^{\gamma} D_{y}^{\delta} a(x+i y, \xi+i \eta)\right| \\
& \leq C m(x, \xi)\left(C \rho_{a}\right)^{-|\alpha+\beta+\gamma+\delta|} e^{-c_{0}(\langle x\rangle /|y|)^{1 /(d-1)}}\langle\xi\rangle^{-|\alpha|}\langle x\rangle^{-|\beta|}\langle\eta\rangle^{-|\gamma|}\langle y\rangle^{-|\delta|}|\alpha+\beta+\gamma+\delta| !^{d} \\
& \left|\left(\partial_{\xi_{j}}+i \partial_{\eta_{j}}\right) \partial_{\xi}^{\alpha} D_{x}^{\beta} \partial_{\eta}^{\gamma} D_{y}^{\delta} a(x+i y, \xi+i \eta)\right| \\
& \leq C m(x, \xi)\left(C \rho_{a}\right)^{-|\alpha+\beta+\gamma+\delta|} e^{-c_{0}(\langle\xi\rangle /|\eta|)^{1 /(d-1)}}\langle\xi\rangle^{-|\alpha|}\langle x\rangle^{-|\beta|}\langle\eta\rangle^{-|\gamma|}\langle y\rangle^{-|\delta|}|\alpha+\beta+\gamma+\delta| !^{d},
\end{aligned}
$$

for any $x, y, \xi, \eta \in \boldsymbol{R}^{n}$ and $\alpha, \beta, \gamma, \delta \in \boldsymbol{N}^{n}$.

Let $\vartheta(x, \xi) \in \gamma^{d} S\left(\delta_{\vartheta}\langle x\rangle^{\kappa}+\rho_{\vartheta}\langle\xi\rangle^{\kappa}, g_{1}\right)$, where $d \kappa \leq 1, d \geq 1$ and $\kappa<1$. Denote $\vartheta(z, \zeta)$ the almost analytic extension of $\vartheta$. For $x, \xi, z, \zeta \in C^{n}$ we set

$$
\begin{aligned}
& \tilde{\nabla}_{x} \vartheta(x, z, \zeta)=\int_{0}^{1} \nabla_{x} \vartheta(z+t(x-z), \zeta) d t \\
& \tilde{\nabla}_{\xi} \vartheta(x, \xi, \zeta)=\int_{0}^{1} \nabla_{\xi} \vartheta(z, \zeta+t(\xi-\zeta)) d t .
\end{aligned}
$$

Lemma 2.10. Let $\vartheta \in \gamma^{d} S\left(\delta_{\vartheta}\langle x\rangle^{\kappa}+\rho_{\vartheta}\langle\xi\rangle^{\kappa}, g_{1}\right)$, where $d \kappa \leq 1, d \geq 1$ and $\kappa<1$. Then, if $\delta_{\vartheta}$ and $\rho_{\vartheta}$ are small enough, there exists $\Phi(x, z, \zeta)$ and $\Phi^{\prime}(z, \xi, \zeta)$ in $\mathscr{C}^{\infty}\left(C^{3 n}\right)$ satisfying

$$
\begin{array}{r}
\Phi-i \tilde{\nabla}_{x} \vartheta(x, z, \Phi)=\zeta \\
\Phi^{\prime}-i \tilde{\nabla}_{\xi} \vartheta\left(\Phi^{\prime}, \xi, \zeta\right)=z,
\end{array}
$$

for all $x, \xi, z, \zeta \in \boldsymbol{C}^{n}$ and

$$
\begin{aligned}
\left|D_{\zeta}^{\alpha} D_{x}^{\beta} D_{z}^{\gamma}(\Phi(x, z, \zeta)-\zeta)\right| & \leq C_{\Phi}\left(\rho_{\vartheta}+\delta_{\vartheta}\right) \rho_{\Phi}^{-|\alpha+\beta+\gamma|}|\alpha+\beta+\gamma| !^{d}\langle\zeta\rangle^{\kappa-|\alpha|} \\
\left|D_{\zeta}^{\alpha} D_{z}^{\beta} D_{\zeta}^{\gamma}\left(\Phi^{\prime}(z, \zeta, \xi)-z\right)\right| & \leq C_{\Phi^{\prime}}\left(\rho_{\vartheta}+\delta_{\vartheta}\right) \rho_{\Phi^{\prime}}^{-|\alpha+\beta+\gamma|}|\alpha+\beta+\gamma| !^{d}\langle z\rangle^{\kappa-|\beta|} \\
\left|\bar{\partial}_{\zeta} D_{\zeta}^{\alpha} D_{x}^{\beta} D_{z}^{\gamma} \Phi(x, z, \zeta)\right| & \leq C_{\Phi}\left(\rho_{\vartheta}+\delta_{\vartheta}\right) e^{-(|\operatorname{Re} \zeta| /|\operatorname{Im} \zeta|)^{1 /(d-1)}} \rho_{\Phi}^{-|\alpha+\beta+\gamma|}|\alpha+\beta+\gamma| !^{d}\langle\zeta\rangle^{\kappa-|\alpha|} \\
\left|\bar{\partial}_{z_{j}} D_{\xi}^{\alpha} D_{z}^{\beta} D_{\zeta}^{\gamma} \Phi^{\prime}(x, z, \zeta)\right| & \leq C_{\Phi^{\prime}}\left(\rho_{\vartheta}+\delta_{\vartheta}\right) e^{-(|\operatorname{Re} \zeta| /|\operatorname{Im} \zeta|)^{1 /(d-1)}} \rho_{\Phi^{\prime}}^{-|\alpha+\beta+\gamma|}|\alpha+\beta+\gamma| !^{d}\langle z\rangle^{\kappa-|\alpha|},
\end{aligned}
$$

for all $x, \xi, z, \zeta \in C^{n}$ and $\alpha, \beta, \gamma \in N^{n}$. Moreover $\Phi(x, \xi)=\Phi(x, x, \xi)$ and $\Phi^{\prime}(x, \xi)=$ $\Phi^{\prime}(x, \xi, \xi)$ satisfy

$$
\begin{gathered}
\left|D_{\xi}^{\alpha} D_{x}^{\beta}(\Phi(x, \xi)-\xi)\right| \leq C_{\Phi}\left(\rho_{\vartheta}\langle\xi\rangle^{\kappa}\langle x\rangle^{-1}+\delta_{\vartheta}\langle x\rangle^{\kappa-1}\right)\langle\xi\rangle^{-|\alpha|}\langle x\rangle^{-|\beta|} \rho_{\Phi}^{-|\alpha+\beta|}|\alpha+\beta| !^{d} \\
\left|D_{\xi}^{\alpha} D_{x}^{\beta}\left(\Phi^{\prime}(x, \xi)-x\right)\right| \leq C_{\Phi^{\prime}}\left(\rho_{\vartheta}\langle\xi\rangle^{\kappa-1}+\delta_{\vartheta}\langle x\rangle^{\kappa}\langle\xi\rangle^{-1}\right)\langle\xi\rangle^{-|\alpha|}\langle x\rangle^{-|\beta|} \rho_{\Phi^{\prime}}^{-|\alpha+\beta|}|\alpha+\beta| !^{d},
\end{gathered}
$$

for all $x, \xi \in \boldsymbol{R}^{n}$ and $\alpha, \beta, \gamma \in \boldsymbol{N}^{n}$. 
Proposition 2.11. Let $\left\{p_{k}(x, \xi)\right\}_{k \in N}$ be a sequence of symbols such that

$$
\left|D_{\xi}^{\alpha} D_{x}^{\beta} p_{k}(x, \xi)\right| \leq C k !^{d^{\prime}}|\alpha| !^{s}|\beta| !^{d} \rho^{-k-|\alpha+\beta|}\langle\xi\rangle^{-k-|\alpha|}\langle x\rangle^{-k-|\beta|} .
$$

Then there is a $p(x, \xi) \in \gamma^{d, s} S\left(m, g_{1}\right)$ such that

$$
\left|D_{\xi}^{\alpha} D_{x}^{\beta}\left(p(x, \xi)-\sum_{k=0}^{N-1} p_{k}(x, \xi)\right)\right| \leq C N !^{d^{\prime}}|\alpha| !^{s}|\beta| !^{d} \rho^{-N-|\alpha+\beta|}\langle\xi\rangle^{-N-|\alpha|}\langle x\rangle^{-N-|\beta|} .
$$

This result has been given in [21] in the case $d^{\prime}=1, d=s$. We can prove the case $d^{\prime}>1$ and $d \neq s$ in the same way.

\subsection{Pseudodifferential operators.}

To $a \in \mathscr{A}_{\rho, \delta}^{\kappa}$, one can canonically associate a pseudodifferential operator defined by standard oscillatory integrals

$$
a(x, D) u(x)=\text { os- } \int_{\boldsymbol{R}^{n}} e^{i x \xi} a(x, \xi) \hat{u}(\xi) \bar{d} \xi .
$$

We have the following result:

Proposition 2.12. If $|\rho|,|\delta|,\left|\rho^{\prime}\right|,\left|\delta^{\prime}\right|$ are small enough, $a \in \mathscr{A}_{\rho, \delta}^{\kappa}$ acts continuously from $H_{\rho^{\prime}, \delta^{\prime}}^{\kappa}$ to $H_{\rho^{\prime}-\rho, \delta^{\prime}-\delta}^{\kappa}$.

We write $a_{1} \equiv a_{2}$ if $a_{1}-a_{2} \in \mathscr{A}_{\rho, \delta}^{\kappa}$, for some $\rho<0$, since, by Proposition 2.4, $\left(a_{1}-a_{2}\right) u$ is a Gevrey function, if $u \in L^{2}$. We recall also the following result on multiple product of operators.

Proposition 2.13. Let $r_{j}(x, \zeta) \in \mathscr{A}_{0,0}^{\kappa}$, for $j=1,2, \ldots, v$, and put

$$
q_{v}(x, D)=r_{1}(x, D) r_{2}(x, D) \cdots r_{v}(x, D) .
$$

Then the symbol $q_{v}(x, \zeta)$ belongs to $\mathscr{A}_{0,0}^{\kappa}$ and satisfies

$$
\left|q_{v(\beta)}^{(\alpha)}(x, \zeta)\right| \leq C^{v} \prod_{j=1}^{v} C_{j} \rho^{-|\alpha+\beta|}|\alpha| !^{s}|\beta| !^{d}
$$

for $(x, \zeta) \in \boldsymbol{R}^{2 n}, \alpha, \beta \in \boldsymbol{N}^{n}$, where $C$ is independent of $v$ and $\rho=\min _{j=1, \ldots, v}\left\{\rho_{j} / 4\right\}$. (Here $C_{j}$ and $\rho_{j}$ are the constants $C$ and $\rho$ in (2.2) corresponding to $r_{j}$.)

\subsection{Fourier integral operators.}

Let $\vartheta(x, \xi) \in \gamma^{d} S\left(\rho_{\vartheta}\langle\xi\rangle^{\kappa}+\delta_{\vartheta}\langle x\rangle^{\kappa}, g_{1}\right)$, with $\rho_{\vartheta}, \delta_{\vartheta} \geq 0, d \kappa \leq 1, d \geq 1$ and $\kappa<1$, let

$$
\varphi(x, \xi)=x \cdot \xi-i \vartheta(x, \xi) .
$$

For $a \in \mathscr{A}_{0,0}^{\kappa}$ and a phase $\varphi(x, \xi)$ as above, let

$$
a_{\varphi}(x, D) u(x)=\int_{\boldsymbol{R}^{n}} e^{i \varphi(x, \xi)} a(x, \xi) \hat{u}(\xi) \bar{d} \xi
$$

for $u \in H_{\varepsilon_{0}, \varepsilon_{0}}^{\kappa}$.

Putting $p(x, \xi)=a(x, \xi) e^{\theta(x, \xi)}$, we can see that $p(x, \xi) \in \mathscr{A}_{\rho_{\vartheta}, \delta_{\vartheta}}^{\kappa}$. Therefore $a_{\varphi}$ can be regarded as a pseudodifferential operators, with symbol $p(x, \xi)=a(x, \xi) e^{\vartheta(x, \xi)}$. By 
Proposition 2.12, if $\rho, \delta, \rho_{\vartheta}, \delta_{\vartheta}$ are sufficiently small, $a_{\varphi}(x, D)$ acts continuously from $H_{\rho, \delta}^{\kappa}$ to $H_{\rho-\rho_{\vartheta}, \delta-\delta_{\vartheta}}^{\kappa}$. For $a=1$, one writes

$$
\begin{aligned}
I_{\varphi}(x, D) u(x) & =\int_{\boldsymbol{R}^{n}} e^{i \varphi(x, \xi)} \hat{u}(\xi) \bar{d} \xi \\
I_{\varphi}^{R}(x, D) u(x) & =\int_{\boldsymbol{R}^{n}} e^{i x \xi} \bar{d} \xi \int_{\boldsymbol{R}^{n}} e^{i \varphi(y, \xi)} u(y) d y .
\end{aligned}
$$

Proposition 2.14. If $\rho_{\vartheta}, \delta_{\vartheta}$ are small then $I_{\varphi} \circ I_{\varphi}^{R}\left(\right.$ resp. $\left.I_{\varphi}^{R} \circ I_{\varphi}\right)$ are $L^{2}$-bounded invertible operators, and

$$
I_{\varphi}^{-1}=I_{\varphi}^{R} J=J^{\prime} I_{\varphi}^{R}
$$

for some $J$ and $J^{\prime}$ bounded on $L^{2}$.

Proposition 2.15. Let $a \in \gamma^{d} S\left(\langle x\rangle^{m}\langle\xi\rangle^{\ell}, g_{1}\right), \vartheta \in \gamma^{d} S\left(\langle x\rangle^{\sigma}\langle\xi\rangle^{\delta}, g_{1}\right), 0 \leq \sigma, \delta \leq 1$, $\sigma+\delta=\kappa$ and let $\varphi=x \xi-i \varepsilon \vartheta(x, \xi)$, with $d \kappa \leq 1, d \geq 1$ and $\kappa<1$. If $\varepsilon$ is small enough,

$$
\begin{aligned}
& I_{\varphi}(x, D) a(x, D) I_{\varphi}^{-1}(x, D) \\
& I_{\varphi}^{-1}(x, D) a(x, D) I_{\varphi}(x, D) \\
& a_{\varphi}(x, D) I_{\varphi}^{-1}(x, D) \\
& I_{\varphi}^{-1}(x, D) a_{\varphi}(x, D) \\
& a(x, D) I_{\varphi}(x, D)
\end{aligned}
$$

are pseudodifferential operators, which symbols (modulo $\left.\mathscr{A}_{\rho, \delta}^{\kappa}\right)$ are given respectively by

$$
\begin{aligned}
& a\left(x-i \nabla_{\xi} \vartheta(x, \Phi), \xi+i \nabla_{x} \vartheta(x, \Phi)\right)+a_{1} \\
& a\left(x+i \nabla_{\xi} \vartheta\left(\Phi^{\prime}, \xi\right), \xi-i \nabla_{x} \vartheta\left(\Phi^{\prime}, \xi\right)\right)+a_{2} \\
& a\left(x, \xi+i \nabla_{x} \vartheta(x, \Phi)\right)+a_{3} \\
& a\left(x+i \nabla_{\xi} \vartheta\left(\Phi^{\prime}, \xi\right), \xi\right)+a_{4} \\
& e^{\theta(x, \xi)} \sum_{\gamma} \frac{1}{\gamma !} D_{y}^{\gamma} D_{\eta}^{\gamma}\left[a\left(x+y-i \tilde{\nabla}_{\xi} \theta(x, \xi, \eta), \xi\right)\right] \begin{array}{c}
y=x \\
\eta=\xi
\end{array}
\end{aligned}
$$

where $a_{1}, a_{2}, a_{3}, a_{4}$ belong to $\gamma^{d} S\left(\langle x\rangle^{m-1}\langle\xi\rangle^{\ell-1}, g_{1}\right), \Phi(x, \xi)=\Phi(x, x, \xi)$ and $\Phi^{\prime}(x, \xi)=$ $\Phi^{\prime}(x, \xi, \xi)$ are defined in (2.4) and (2.5).

\subsection{Phase function.}

Let $e(x, \xi)=\sqrt{1+a_{2}(x, \xi)}$. For $\varepsilon, M>0$, we consider

$$
\begin{aligned}
\vartheta_{0}^{(\varepsilon, M)}(x, \xi)= & \frac{\theta(x, \xi)}{\langle x\rangle^{1-\sigma} e(x, \xi)^{1-\delta}} \Phi_{0}\left(\frac{\theta(x, \xi)}{\langle x\rangle e(x, \xi) \varepsilon}\right) \\
& +e(x, \xi)^{\delta-\sigma} f(\theta(x, \xi))\left[\Phi_{+}\left(\frac{\theta(x, \xi)}{\langle x\rangle e(x, \xi) \varepsilon}\right)-\Phi_{-}\left(\frac{\theta(x, \xi)}{\langle x\rangle e(x, \xi) \varepsilon}\right)\right]
\end{aligned}
$$

and 


$$
\vartheta_{1}^{(\varepsilon, M)}(t, x, \xi)=\vartheta_{0}^{(\varepsilon, M)}(x, \xi) \Phi_{0}\left(\frac{\langle x\rangle}{M e(x, \xi)}\right)+t e(x, \xi)^{\sigma+\delta}\left[1-\Phi_{0}\left(\frac{\langle x\rangle}{M e(x, \xi)}\right)\right],
$$

where $f(\tau)=\int_{0}^{\tau}\left(1+s^{2}\right)^{(\sigma-1) / 2} d s$, and $\Phi_{ \pm}(\tau)=\chi( \pm \tau), \Phi_{0}(\tau)=1-\Phi_{+}(\tau)-\Phi_{-}(\tau)$ and $\chi(\tau) \in \gamma^{d}(\boldsymbol{R})$ such that $\chi(\tau)=1$ for $\tau \geq 1, \chi(\tau)=0$ for $\tau \leq 1 / 2$, and $\chi^{\prime}(\tau) \geq 0$.

Proposition 2.16. The function $\vartheta_{1}^{(\varepsilon, M)}$ belongs to $\mathscr{C}^{1}\left([0, T] ; \gamma^{d} S\left(\langle x\rangle^{\sigma}\langle\xi\rangle^{\delta}, g_{1}\right)\right)$ and

$$
\vartheta_{1}^{(\varepsilon, M)}(0, x, \xi)=\vartheta_{0}^{(\varepsilon, M)}(x, \xi)=c(x, \xi) \frac{\theta(x, \xi)}{\langle x\rangle^{1-\sigma} e(x, \xi)^{1-\delta}}
$$

where $c(x, \xi)$ belongs to $\gamma^{d} S\left(1, g_{1}\right)$, and satisfies $c(x, \xi) \geq c_{0}>0$. There are $\varepsilon_{0}>0$, $M_{0}>0, K>0, c_{0}>0$ such that

$$
\left(\partial_{t}+H_{a_{2}}\right) \vartheta_{1}^{(\varepsilon, M)}(t, x, \xi) \geq c_{0}\left(\langle\xi\rangle^{\kappa}+\langle x\rangle^{2 \sigma-2}\langle\xi\rangle^{2 \delta}+\langle x\rangle^{\kappa-1}\langle\xi\rangle\right),
$$

for all $|\varepsilon| \leq \varepsilon_{0}, M \geq M_{0}$, all $x, \xi \in \boldsymbol{R}^{n}$ with $|\xi| \geq K$ and $0<t \leq T$.

\subsection{Criterion for $L^{2}$ well-posedness.}

Proposition 2.17. Consider the following Cauchy problem

$$
\left\{\begin{array}{l}
\partial_{t} u(t, x)-i a(x, D) u(t, x)-b(t, x, D) u(t, x)=f(t, x) \\
u(0, x)=u_{0}(x)
\end{array}\right.
$$

Assume that $a(x, \xi) \in S_{1,0}^{2}$ has real value and $b(t, x, \xi) \in C^{0}\left([0, T] ; S_{1,0}^{1}\right)$ are such that

$$
\operatorname{Re} b(t, x, \xi)-\frac{1}{2} \sum_{j, k=1}^{n} a_{x j \xi_{k}}(x, \xi) \leq C
$$

for $x \in \boldsymbol{R}^{n},|x| \geq K$, and $\xi \in \boldsymbol{R}^{n}$. Assume moreover that there exists $\theta(x, \xi)$ verifying Assumption I-(3). Then, if $u_{0} \in L^{2}$ and $f \in C^{0}\left([0, T] ; L^{2}\right)$ there exists a unique solution $u \in C^{0}\left([0, T] ; L^{2}\right) \cap C^{1}\left([0, T] ; H^{-2}\right)$ of the Cauchy problem (2.17).

\section{Fundamental solution.}

In this section we shall construct the fundamental solution of the Cauchy problem for $p(t, x, \xi) \in \mathscr{C}^{0}\left([-T, T] ; \gamma^{d, s} S(m(x, \xi), g)\right)$, that is, $p(t, x, \xi)$ satisfies

$$
\left|p_{(\beta)}^{(\alpha)}(t, x, \xi)\right| \leq C_{p} \rho_{p}^{-|\alpha+\beta|}|\alpha| !^{s}|\beta| !^{d} m(x, \xi)\langle\xi\rangle^{-|\alpha|}\langle x\rangle^{-|\beta|},
$$

for $x, \xi \in \boldsymbol{R}^{n}, \alpha, \beta \in \boldsymbol{N}^{n}$, where $m(x, \xi)$ satisfies

$$
m(x, \xi) \leq C\langle x\rangle^{\kappa}\langle\xi\rangle^{\kappa},
$$

for some $C>0$ and $\kappa \in] 0,1[$. Calculations are similar to those in [24, Proposition 3.8] and $[\mathbf{2 1}, \S 1]$.

For $T>0$ we consider the following Cauchy problem,

$$
\left\{\begin{array}{l}
\left.\frac{d}{d t} E(t)=\rho p(t, x, D) E(t), \quad t \in\right]-T, T[ \\
E(0)=I .
\end{array}\right.
$$


Proposition 3.1. Let $p(t, x, \xi)$ as above, if $\rho>0$ is small enough, then we can construct the fundamental solution $E(t)$ of (3.3) as a Fourier integral operator:

$$
E(t)=I_{\psi(t)}(e(t, x, D)+r(t, x, D))
$$

where $\psi(t, x, \xi)=x \cdot \xi-i \rho \theta(t, x, \xi)$, with $\theta(t, x, \xi) \in \mathscr{C}^{1}\left([-T, T] ; \gamma^{d, s} S(m(x, \xi), g)\right)$ is such that $\theta(0, x, \xi)=0$ and

$$
\partial_{t} \theta(t, x, \xi)-p\left(t, x, \xi-i \nabla_{x} \theta(t, x, \xi)\right) \in \mathscr{C}^{0}\left([-T, T] ; \gamma^{d, s} S(1, g)\right),
$$

$e(t, x, \xi) \in \mathscr{C}^{1}\left([-T, T] ; \gamma^{d, s} S(1, g)\right)$ and $r(t, x, \xi) \in \mathscr{C}^{1}\left([-T, T] ; \gamma^{d, s} S\left(e^{-\varepsilon(\langle x\rangle\langle\xi\rangle)^{1 /(d+s-1)}}, g_{0}\right)\right)$.

Moreover, if $p(x, D)$ does not depend on $t$, then $E(t)^{-1}=E(-t)$, and

$$
E(t) a(x, D) E(-t)=a(x, D)+\int_{0}^{t} E(s)[p, a] E(-s) d s, \quad t \in[-T, T]
$$

where $[\cdot, \cdot]$ stands for a commutator.

ProOF. First of all we construct the phase function $\theta(t, x, \xi)$ as follows,

$$
\theta(t, x, \xi)=\sum_{j=0}^{N-1} \theta_{j}(t, x, \xi)
$$

with $\theta_{j} \in \mathscr{C}^{1}\left([-T, T] ; \gamma^{d, s} S\left(m^{j+1}\langle x\rangle^{-j}\langle\xi\rangle^{-j}, g\right)\right)$. By the definition of almost analytic extension, we have

$$
\begin{aligned}
p(t, x, \xi & \left.-i \nabla_{x}\left(\theta_{0}+\cdots+\theta_{N-1}\right)\right)-\sum_{|\alpha|<N} \frac{1}{\alpha !} p^{(\alpha)}(t, x, \xi)\left(-i \nabla_{x}\left(\theta_{0}+\cdots+\theta_{N-1}\right)\right)^{\alpha} \\
= & \sum_{|\alpha|<N} \frac{1}{\alpha !} p^{(\alpha)}(t, x, \xi)\left(-i \nabla_{x}\left(\theta_{0}+\cdots+\theta_{N-1}\right)\right)^{\alpha}\left[\chi\left(b_{\alpha} \frac{\left|\nabla_{x}\left(\theta_{0}+\cdots+\theta_{N-1}\right)\right|}{\langle\xi\rangle}\right)-1\right] \\
& +\sum_{|\alpha| \geq N} \frac{1}{\alpha !} p^{(\alpha)}(t, x, \xi)\left(-i \nabla_{x}\left(\theta_{0}+\cdots+\theta_{N-1}\right)\right)^{\alpha} \chi\left(b_{\alpha} \frac{\left|\nabla_{x}\left(\theta_{0}+\cdots+\theta_{N-1}\right)\right|}{\langle\xi\rangle}\right) \\
= & \sum_{|\alpha|<N} \frac{1}{\alpha !} p_{\alpha}^{\prime}(t, x, \xi)+\sum_{|\alpha| \geq N} \frac{1}{\alpha !} p_{\alpha}^{\prime \prime}(t, x, \xi) .
\end{aligned}
$$

Since $p$ satisfies (3.1), we can see that

$$
p_{\alpha}^{\prime \prime}(t, x, \xi) \in \mathscr{C}^{1}\left([-T, T] ; \gamma^{d, s} S\left(m^{N+1}(x, \xi)\langle x\rangle^{-N}\langle\xi\rangle^{-N}, g\right)\right), \quad \text { if }|\alpha| \geq N .
$$

On the other hand, on $\operatorname{supp}\left[\chi\left(b_{\alpha}\left(\left|\nabla_{x}\left(\theta_{0}+\cdots+\theta_{N-1}\right)\right| /\langle\xi\rangle\right)-1\right]\right.$ we have $m(x, \xi) /$ $\langle x\rangle\langle\xi\rangle \geq C$. Then

$$
p_{\alpha}^{\prime}(t, x, \xi) \in \mathscr{C}^{1}\left([-T, T] ; \gamma^{d, s} S\left(m^{N+1}(x, \xi)\langle x\rangle^{-N}\langle\xi\rangle^{-N}, g\right)\right), \quad \text { if }|\alpha|<N .
$$

Noting that 


$$
\begin{aligned}
& \frac{1}{\alpha !}\left(-i \nabla_{x}\left(\theta_{0}+\cdots+\theta_{N-1}\right)\right)^{\alpha} \\
& \quad=\sum_{\beta^{0}+\cdots+\beta^{N-1}=\alpha} C_{\beta^{0} \cdots \beta^{N-1}}^{\alpha}\left(-i \nabla_{x} \theta_{0}\right)^{\beta^{0}}\left(-i \nabla_{x} \theta_{1}\right)^{\beta^{1}} \cdots\left(-i \nabla_{x} \theta_{N-1}\right)^{\beta^{N-1}}
\end{aligned}
$$

and

$$
\begin{aligned}
& p^{(\alpha)}(t, x, \xi)\left(-i \nabla_{x} \theta_{0}\right)^{\beta^{0}}\left(-i \nabla_{x} \theta_{1}\right)^{\beta^{1}} \cdots\left(-i \nabla_{x} \theta_{N-1}\right)^{\beta^{N-1}} \\
& \quad \in \mathscr{C}^{1}\left([-T, T] ; \gamma^{d, s} S\left(m^{1+\sum_{k=0}^{N-1}(k+1)\left|\beta^{k}\right|}\langle x\rangle^{-\sum_{k=1}^{N-1}(k+1)\left|\beta^{k}\right|}\langle\xi\rangle^{-\sum_{k=1}^{N-1} k\left|\beta^{k}\right|-|\alpha|}, g\right)\right),
\end{aligned}
$$

we get

$$
\begin{aligned}
& p\left(t, x, \xi-i \nabla_{x}\left(\theta_{0}+\cdots+\theta_{N-1}\right)\right) \\
& -\sum_{j=0}^{N-1} \sum_{\left|\beta^{0}\right|+2\left|\beta^{1}\right|+\cdots+j\left|\beta^{j-1}\right|=j} C_{\beta^{0} \cdots \beta^{N-1}}^{\alpha} p^{(\alpha)}(t, x, \xi)\left(-i \nabla_{x} \theta_{0}\right)^{\beta^{0}} \cdots\left(-i \nabla_{x} \theta_{j-1}\right)^{\beta^{j-1}} \\
& \in \mathscr{C}^{1}\left([-T, T] ; \gamma^{d, s} S\left(m^{N+1}(x, \xi)\langle x\rangle^{-N}\langle\xi\rangle^{-N}, g\right)\right) \text {. }
\end{aligned}
$$

Therefore we can obtain $\theta_{j}(x, \xi)$ by solving the following equations

$$
\left\{\begin{array}{l}
\partial_{t} \theta_{0}(t, x, \xi)=p(t, x, \xi) \\
\theta_{0}(0, x, \xi)=0
\end{array}\right.
$$

for $j=0$, and

$$
\left\{\begin{aligned}
\partial_{t} \theta_{j}(t, x, \xi)= & \sum_{\left|\beta^{0}\right|+2\left|\beta^{1}\right|+\cdots+j\left|\beta^{j-1}\right|=j} C_{\beta^{0} \cdots \beta^{j-1}}^{\alpha} p^{(\alpha)}(t, x, \xi) \\
& \times\left(-i \nabla_{x} \theta_{0}\right)^{\beta^{0}}\left(-i \nabla_{x} \theta_{1}\right)^{\beta^{1}} \cdots\left(-i \nabla_{x} \theta_{j-1}\right)^{\beta^{j-1}} \\
\theta_{j}(0, x, \xi)= & 0
\end{aligned}\right.
$$

for $j=1, \ldots, N-1$. If we take $N$ such that $\kappa-(1-\kappa) N \leq 0$, then $\theta=\sum_{j=0}^{N-1} \theta_{j}$ satisfies (3.5). We note that $\theta(t, z, \xi), z=x+i y$, an almost analytic extension of $\theta(t, x, \xi)$, also satisfies

$$
\partial_{t} \theta(t, z, \xi)-p\left(t, z, \xi-i \nabla_{x} \theta(t, z, \xi)\right) \in \mathscr{C}^{0}\left([-T, T] ; \mathscr{A}_{-\varepsilon_{0},-\varepsilon_{0}}^{\kappa}\right)
$$

if $|y| \leq C\langle\xi\rangle^{\kappa-1}\langle x\rangle^{\kappa}$ and when $p(t, x, \xi) \geq c_{0}\langle\xi\rangle^{\kappa}$, then

$$
\operatorname{Re} \theta(t, x, \xi) \geq c_{1} t\langle\xi\rangle^{\kappa}
$$

Now we can construct the solution of (3.3). For $\theta(t, x, \xi)$ satisfying (3.5) and consequently (3.7) we denote $\varphi(t)=x \xi-i \rho \theta(t, x, \xi)$. We seek a solution $E(t)$ as a following form,

$$
E(t)=I_{\varphi(t)}(x, D) e(t, x, D) .
$$


If $\rho$ is small, we have the inverse operator $I_{\varphi(t)}(x, D)^{-1}$ from Proposition 2.14. Therefore if $E(t)$ satisfies (3.3), then $e(t, x, D)$ satisfies

$$
\frac{d}{d t} e(t, x, D)=I_{\varphi(t)}(x, D)^{-1}\left[\rho p(t, x, D) I_{\varphi(t)}(x, D)-\partial_{t} I_{\varphi(t)}(x, D)\right] e(t, x, D) .
$$

Noting that $\partial_{t} I_{\varphi(t)}(x, D)=\left(\partial_{t} \theta\right)_{\varphi(t)}$, we can see from (2.12) that

$$
\sigma\left(I_{\varphi(t)}^{-1} \partial_{t} I_{\varphi(t)}\right)(x, \xi)=\frac{\partial \theta}{\partial t}\left(t, \Phi^{\prime}(t, x, \xi), \xi\right)+\theta_{1}(t, x, \xi)+r_{1}(t, x, \xi),
$$

where $\Phi^{\prime}$ satisfies

$$
\Phi^{\prime}(t, x, \xi)=x+i \nabla_{\xi} \theta\left(t, \Phi^{\prime}(t, x, \xi), \xi\right)
$$

$\theta_{1} \in \mathscr{C}^{0}\left([-T, T] ; \gamma^{d, s} S(1, g)\right)$, and $r_{1} \in \mathscr{C}^{0}\left([-T, T] ; \mathscr{A}_{-\varepsilon_{0},-\varepsilon_{0}}^{\kappa}\right)$. Moreover, from $(2.10)$

$$
\begin{aligned}
& \sigma\left(I_{\varphi(t)}(x, D)^{-1} p(t, x, D) I_{\varphi(t)}(x, D)\right)(x, \xi) \\
& \quad=p\left(t, \Phi^{\prime}(t, x, \xi), \xi-i \nabla_{x} \theta\left(t, \Phi^{\prime}(t, x, \xi)\right)\right)+p_{1}(t, x, \xi)+r_{2}(t, x, \xi)
\end{aligned}
$$

where $p_{1} \in \mathscr{C}^{0}\left([-T, T] ; \gamma^{d, s} S(1, g)\right)$ and $r_{2} \in \mathscr{C}^{0}\left([-T, T] ; \mathscr{A}_{-\varepsilon_{0},-\varepsilon_{0}}^{\kappa}\right)$. Thus from (3.10)(3.12) it follows that taking account of (3.7) and (3.11) we have the following equation for $e(t)=e(t, x, D)$,

$$
\left\{\begin{array}{l}
\frac{d}{d t} e(t)=q(t, x, D) e(t)+r(x, D) \\
e(0)=I
\end{array}\right.
$$

where $q \in \mathscr{C}^{0}\left([-T, T] ; \gamma^{d, s} S(1, g)\right)$ and $r \in \mathscr{C}^{0}\left([-T, T] ; \mathscr{A}_{-\varepsilon_{0},-\varepsilon_{0}}^{\kappa}\right)$. First we construct an asymptotic solution of (3.13) as follows,

$$
e(t, s ; x, \xi)=\sum_{j=0}^{\infty} e_{j}(t, s ; x, \xi)
$$

with $e_{j}(t, s, x, \xi)$ satisfying

$$
\left\{\begin{array}{l}
\left(\partial_{t}-q(t, x, \xi)\right) e_{0}(t, s ; x, \xi)=0 \\
e_{0}(s, s ; x, \xi)=1
\end{array} \text { for } j=0\right.
$$

and

$$
\left\{\begin{array}{l}
\left(\partial_{t}-q(t, x, \xi)\right) e_{j}(t, s ; x, \xi)=P_{j}(t, x, \xi) \\
e_{j}(s, s ; x, \xi)=0
\end{array} \text { for } j \geq 1,\right.
$$

where

$$
P_{j}(t, x, \xi)=\sum_{k=0}^{j-1} \sum_{|\gamma|=j-k} \frac{1}{\gamma !} q^{(\gamma)}(t, x, \xi) e_{k(\gamma)}(t, x, \xi), \quad(j \geq 1) .
$$


Solving the above equations, we get

$$
\begin{aligned}
e_{0}(t, s ; x, \xi) & =e^{\int_{s}^{t} q(\tau, x, \xi) d \tau} \\
e_{j}(t, s ; x, \xi) & =\int_{s}^{t} P_{j}(\tau, x, \xi) e_{0}(t, \tau, x, \xi) d \tau \quad(j \geq 1) .
\end{aligned}
$$

We shall prove that $e_{j}$ verifies the following estimates

$$
\left|\partial_{\xi}^{\alpha} D_{x}^{\beta} e_{j}(t, s ; x, \xi)\right| \leq C_{1}^{j+1} C_{2}^{|\alpha+\beta|} \frac{(|\alpha|+j) !^{s}(|\beta|+j) !^{d}}{j !}\langle x\rangle^{-j-|\beta|}\langle\xi\rangle^{-j-|\alpha|},
$$

for $t, s \in[-T, T], x \in \boldsymbol{R}^{n}, \xi \in \boldsymbol{R}^{n}, \alpha, \beta \in \boldsymbol{N}^{n}, j \in \boldsymbol{N}$. Since $q \in \mathscr{C}^{1}\left([-T, T] ; \gamma^{d, s} S(1, g)\right)$, we have

$$
\left|D_{x}^{\beta} \partial_{\xi}^{\alpha} q(t, x, \xi)\right| \leq C_{0}^{|\alpha+\beta|+1}|\alpha| !^{s}|\beta| !^{d}\langle x\rangle^{-|\beta|}\langle\xi\rangle^{-|\alpha|}
$$

for $x \in \boldsymbol{R}^{n}, \xi \in \boldsymbol{R}^{n}$ and $\alpha, \beta \in \boldsymbol{N}^{n}$. Therefore it follows from (3.15) that $e_{0}(t, s ; x, \xi)$ satisfies

$$
\left|D_{x}^{\beta} \partial_{\xi}^{\alpha} e_{0}(t, s ; x, \xi)\right| \leq C_{0}^{|\alpha+\beta|+1}|\alpha| !^{s}|\beta| !^{d}\langle x\rangle^{-|\beta|}\langle\xi\rangle^{-|\alpha|}
$$

for $t, s \in[-T, T], x \in \boldsymbol{R}^{n}, \xi \in \boldsymbol{R}^{n}$ and $\alpha, \beta \in \boldsymbol{N}^{n}$, modifying $C_{0}$ if necessary. Hence we have (3.17) for $j=0$ if we take $C_{1} \geq C_{0}$ and $C_{2} \geq C_{0}$. Assume that (3.17) is valid for $j-1$. Then we have from (3.14)

$$
\partial_{\xi}^{\alpha} D_{x}^{\beta} P_{j}(t, x, \xi)=\sum_{k=0}^{j-1} \sum_{|\gamma|=j-k} \frac{1}{\gamma !} \sum_{\alpha^{\prime}+\alpha^{\prime \prime}=\alpha} \sum_{\beta^{\prime}+\beta^{\prime \prime}=\beta}\left(\begin{array}{c}
\alpha \\
\alpha^{\prime}
\end{array}\right)\left(\begin{array}{c}
\beta \\
\beta^{\prime}
\end{array}\right) q_{\left(\beta^{\prime}\right)}^{\left(\gamma+\alpha^{\prime}\right)}(t, x, \xi) e_{k\left(\beta^{\prime \prime}+\gamma\right)}^{\left(\alpha^{\prime \prime}\right)}(t, s ; x, \xi) .
$$

We get by use of the assumption of induction (3.17) and (3.18):

$$
\begin{aligned}
\left|\partial_{\xi}^{\alpha} D_{x}^{\beta} P_{j}(t, x, \xi)\right| & \\
\leq & \sum_{k=0}^{j-1} \sum_{|\gamma|=j-k} \frac{1}{\gamma !} \sum_{\alpha^{\prime}+\alpha^{\prime \prime}=\alpha} \sum_{\beta^{\prime}+\beta^{\prime \prime}=\beta}\left(\begin{array}{c}
\alpha \\
\alpha^{\prime}
\end{array}\right)\left(\begin{array}{c}
\beta \\
\beta^{\prime}
\end{array}\right) C_{0}^{\left|\alpha^{\prime}+\beta^{\prime}+\gamma\right|+1}\left|\alpha^{\prime}+\gamma\right| !^{s}\left|\beta^{\prime}\right| !^{d}\langle x\rangle^{-\left|\beta^{\prime}\right|}\langle\xi\rangle^{-\left|\alpha^{\prime}+\gamma\right|} \\
& \times C_{1}^{k+1} C_{2}^{\left|\alpha^{\prime \prime}+\beta^{\prime \prime}+\gamma\right|} \frac{1}{k !}\left(\left|\alpha^{\prime \prime}\right|+k\right) !^{s}\left(\left|\beta^{\prime \prime}+\gamma\right|+k\right) !^{\prime}\langle x\rangle^{-k-\left|\beta^{\prime \prime}+\gamma\right|}\langle\xi\rangle^{-k-\left|\alpha^{\prime \prime}\right|} \\
\leq & \sum_{k=0}^{j-1} \sum_{|\gamma|=j-k} \frac{1}{\gamma !} C_{0}^{|\gamma|+1} C_{1}^{k+1} C_{2}^{|\alpha+\beta+\gamma|} \frac{1}{k !}\left[\sum_{\alpha^{\prime}+\alpha^{\prime \prime}=\alpha}\left(\begin{array}{c}
\alpha \\
\alpha^{\prime}
\end{array}\right)\left(\frac{C_{0}}{C_{2}}\right)^{\left|\alpha^{\prime}\right|}\left|\alpha^{\prime}+\gamma\right| !^{s}\left(\left|\alpha^{\prime \prime}\right|+k\right) !^{s}\right] \\
& \times\left[\sum_{\beta^{\prime}+\beta^{\prime \prime}=\beta}\left(\begin{array}{c}
\beta \\
\beta^{\prime}
\end{array}\right)\left(\frac{C_{0}}{C_{2}}\right)^{\left|\beta^{\prime}\right|}\left|\beta^{\prime}\right| !^{d}\left(\left|\beta^{\prime \prime}+\gamma\right|+k\right) !^{d}\right]\langle x\rangle^{-k-|\gamma+\beta|}\langle\xi\rangle^{-k-|\alpha+\gamma|}
\end{aligned}
$$

Using Lemma 2.2, we have if $C_{2}>C_{0}$ 


$$
\begin{aligned}
\left|\partial_{\xi}^{\alpha} D_{x}^{\beta} P_{j}(t, x, \xi)\right| \leq & \frac{C_{0}^{2}}{\left(C_{2}-C_{0}\right)^{2}} \sum_{k=0}^{j-1} \sum_{|\gamma|=j-k} \frac{1}{\gamma !} C_{0}^{|\gamma|+1} C_{1}^{k+1} C_{2}^{|\alpha+\beta+\gamma|} \frac{1}{k !} \\
& \times(|\alpha+\gamma|+k) !^{s}\left(\begin{array}{c}
|\gamma|+k \\
|\gamma|
\end{array}\right)^{-1}(|\beta+\gamma|+k) !^{d}\langle x\rangle^{-k-|\gamma+\beta|}\langle\xi\rangle^{-k-|\alpha+\gamma|} \\
= & \frac{C_{0}^{3}}{\left(C_{2}-C_{0}\right)^{2}} C_{1}^{j+1} C_{2}^{|\alpha+\beta|} \frac{(|\alpha|+j) !(|\beta|+j) !^{d}}{j !}\langle x\rangle^{-j-|\beta|}\langle\xi\rangle^{-j-|\alpha|} \\
& \times \sum_{k=0}^{j-1}\left(\frac{C_{0} C_{2}}{C_{1}}\right)^{j-k} \sum_{|\gamma|=j-k} \frac{|\gamma| !}{\gamma !} .
\end{aligned}
$$

Now $\sum_{|\gamma|=j-k}|\gamma| ! / \gamma !=(1+\cdots+1)^{j-k}=n^{j-k}$, then

$$
\sum_{k=0}^{j-1}\left(\frac{C_{0} C_{2}}{C_{1}}\right)^{j-k} \sum_{|\gamma|=j-k} \frac{|\gamma| !}{\gamma !}=\sum_{k=0}^{j-1}\left(\frac{n C_{0} C_{2}}{C_{1}}\right)^{j-k} \leq \frac{n C_{0} C_{2}}{C_{1}-n C_{0} C_{2}}
$$

if $C_{1}>n C_{0} C_{2}$ and consequently

$$
\begin{aligned}
\left|\partial_{\xi}^{\alpha} D_{x}^{\beta} P_{j}(t, x, \xi)\right| \leq & \frac{n C_{0}^{4} C_{2}}{\left(C_{2}-C_{0}\right)^{2}\left(C_{1}-n C_{0} C_{2}\right)} C_{1}^{j+1} C_{2}^{|\alpha+\beta|} \\
& \times \frac{(|\alpha|+j) ! s(|\beta|+j) !^{d}}{j !}\langle x\rangle^{-j-|\beta|}\langle\xi\rangle^{-j-|\alpha|} .
\end{aligned}
$$

From (3.16) we get

$$
\left|\partial_{\xi}^{\alpha} D_{x}^{\beta} e_{j}(t, s ; x, \xi)\right| \leq \int_{s}^{t} \sum_{\alpha^{\prime}+\alpha^{\prime \prime}=\alpha} \sum_{\beta^{\prime}+\beta^{\prime \prime}=\beta}\left(\begin{array}{c}
\alpha \\
\alpha^{\prime}
\end{array}\right)\left(\begin{array}{c}
\beta \\
\beta^{\prime}
\end{array}\right)\left|p_{j\left(\beta^{\prime}\right)}^{\left(\alpha^{\prime}\right)}(s, x, \xi) e_{0\left(\beta^{\prime \prime}\right)}^{\left(\alpha^{\prime \prime}\right)}(t, s ; x, \xi)\right| d s .
$$

Hence we get from (3.19) and (3.20)

$$
\begin{aligned}
& \left|\partial_{\xi}^{\alpha} D_{x}^{\beta} e_{j}(t, s ; x, \xi)\right| \\
& \leq \frac{n C_{0}^{4} C_{2} T}{\left(C_{2}-C_{0}\right)^{2}\left(C_{1}-n C_{0} C_{2}\right)} \sum_{\alpha^{\prime}+\alpha^{\prime \prime}=\alpha} \sum_{\beta^{\prime}+\beta^{\prime \prime}=\beta}\left(\begin{array}{c}
\alpha \\
\alpha^{\prime}
\end{array}\right)\left(\begin{array}{c}
\beta \\
\beta^{\prime}
\end{array}\right) C_{1}^{j+1} C_{2}^{\left|\alpha^{\prime}+\beta^{\prime}\right|} \\
& \times \frac{\left(\left|\alpha^{\prime}\right|+j\right) ! !^{s}\left(\left|\beta^{\prime}\right|+j\right) !^{d}}{j !}\langle x\rangle^{-j-\left|\beta^{\prime}\right|}\langle\xi\rangle^{-j-\left|\alpha^{\prime}\right|} C_{0}^{\left|\alpha^{\prime \prime}+\beta^{\prime \prime}\right|+1}\left|\alpha^{\prime \prime}\right| !^{s}\left|\beta^{\prime \prime}\right| !^{d}\langle x\rangle^{-\left|\beta^{\prime \prime}\right|}\langle\xi\rangle^{-\left|\alpha^{\prime \prime}\right|} \\
& =\frac{n C_{0}^{4} C_{2} T}{\left(C_{2}-C_{0}\right)^{2}\left(C_{1}-n C_{0} C_{2}\right)} C_{1}^{j+1} C_{2}^{|\alpha+\beta|} \frac{1}{j !}\langle x\rangle^{-j-|\beta|}\langle\xi\rangle^{-j-|\alpha|} \\
& \times\left[\sum_{\alpha^{\prime}+\alpha^{\prime \prime}=\alpha}\left(\begin{array}{c}
\alpha \\
\alpha^{\prime}
\end{array}\right)\left(\frac{C_{0}}{C_{2}}\right)^{\left|\alpha^{\prime \prime}\right|}\left(\left|\alpha^{\prime}\right|+j\right) !^{s}\left|\alpha^{\prime \prime}\right| !^{s}\right]\left[\sum_{\beta^{\prime}+\beta^{\prime \prime}=\beta}\left(\begin{array}{c}
\beta \\
\beta^{\prime}
\end{array}\right)\left(\frac{C_{0}}{C_{2}}\right)^{\left|\beta^{\prime \prime}\right|}\left(\left|\beta^{\prime}\right|+j\right) !^{d}\left|\beta^{\prime \prime}\right| !^{d}\right] \\
& \leq \frac{n C_{0}^{4} C_{2}^{2} T}{\left(C_{2}-C_{0}\right)^{4}\left(C_{1}-n C_{0} C_{2}\right)} C_{1}^{j+1} C_{2}^{|\alpha+\beta|+1} \frac{(|\alpha|+j) !^{s}(|\beta|+j) !^{d}}{j !}\langle x\rangle^{-j-|\beta|}\langle\xi\rangle^{-j-|\alpha|},
\end{aligned}
$$


where we have again used Lemma 2.2. We obtain (3.17), if we choose $C_{1}$ and $C_{2}$ such that

$$
\frac{n C_{0}^{4} C_{2}^{2} T}{\left(C_{2}-C_{0}\right)^{4}\left(C_{1}-n C_{0} C_{2}\right)} \leq 1 .
$$

Next we shall construct the exact solution $e(t)$ of the equation of (3.13). It follows from (3.17) that applying Proposition 2.11 to $\left\{e_{j}\right\}$ we can construct $\tilde{e}(t, s ; x, \xi) \in$ $\mathscr{C}^{1}\left([-T, T]^{2} ; \gamma^{d, s}\left(1, g_{1}\right)\right)$ satisfying

$$
\begin{aligned}
& \left|\partial_{\xi}^{\alpha} D_{x}^{\beta}\left(\tilde{e}(t, s ; x, \xi)-\sum_{j=0}^{N} e_{j}(t, s ; x, \xi)\right)\right| \\
& \quad \leq C_{3}^{1+N+|\alpha+\beta|} N !^{d+s-1}|\alpha| !^{s}|\beta| !^{d}\langle x\rangle^{-N-|\alpha|}\langle\xi\rangle^{-N-|\beta|}
\end{aligned}
$$

for $t \in[-T, T], x, \xi \in \boldsymbol{R}^{n}, \alpha, \beta \in \boldsymbol{N}^{n}$ and for any non negative integer $N$. From (3.13) and (3.21) we obtain the following relation,

$$
\left\{\begin{array}{l}
\left(\partial_{t}-q(t, x, D)\right) \tilde{e}(t, s ; x, D)=R(t, s ; x, D) \quad|t|<T \\
\tilde{e}(s, s ; x, D)=I
\end{array}\right.
$$

for any $s \in[-T, T]$, where $R(t, s ; x, \xi)$ satisfies

$$
\left|R_{(\beta)}^{(\alpha)}(t, s ; x, \xi)\right| \leq C_{4}^{1+|\alpha+\beta|} e^{-\varepsilon_{0}(\langle x\rangle\langle\xi\rangle)^{1 /(d+s-1)}}|\alpha| !^{s}|\beta| !^{d}
$$

for $t \in[-T, T], x, \xi \in \boldsymbol{R}^{n}, \alpha, \beta \in \boldsymbol{N}^{n}$, where $\varepsilon_{0}>0$.

Now we can construct the exact solution of (3.13) given by

$$
e(t, x, D)=\tilde{e}(t, 0 ; x, D)+\int_{0}^{t} \tilde{e}(t, \tau ; x, D) W(\tau, x, D) d \tau,
$$

where $W(t, x, D)$ satisfies

$$
W(t, x, D)=-R(t, 0 ; x, D)-\int_{0}^{t} R(t, \tau ; x, D) W(\tau, x, D) d \tau .
$$

We can solve the above integral equation as follows

$$
W(t, x, D)=\sum_{j=1}^{\infty} W_{j}(t, x, D),
$$

where

$$
\begin{aligned}
W_{1}(t, x, D) & =-R(t, 0 ; x, D) \\
W_{j}(t, x, D) & =\int_{0}^{t} R(t, \tau ; x, D) W_{j-1}(\tau, x, D) d \tau, \quad(j \geq 2) \\
& =\int_{0}^{t} \int_{0}^{\tau_{1}} \cdots \int_{0}^{\tau_{j-2}}(-1)^{j} R\left(t, \tau_{1}\right) R\left(\tau_{1}, \tau_{2}\right) \cdots R\left(\tau_{j-1}, \tau_{j}\right) R\left(\tau_{j}, 0\right) d \tau_{1} \cdots d \tau_{j-1} .
\end{aligned}
$$

Since we can regard $R(t, s ; x, \xi)$ as an element of $A_{0,0}^{\kappa}$ from (3.22), we can apply 
Proposition 2.13 to the right hand side in (3.26). Then $W_{j}(t, x, \xi)$ satisfies the following estimate from $(2.8)$ and by $(j-1)$-times integrations,

$$
\left|W_{j(\beta)}^{(\alpha)}(t, x, \xi)\right| \leq C_{4}^{1+|\alpha+\beta|} \frac{(C|t|)^{j-1}}{(j-1) !}|\alpha| !^{s}|\beta| !^{d},
$$

for any $j \geq 1, t \in[-T, T], x, \xi \in \boldsymbol{R}^{n}, \alpha, \beta \in \boldsymbol{N}^{n}$. Therefore $W(t, x, \xi)$ given by (3.25) converges in $\mathscr{A}_{0,0}^{\kappa}$. On the other hand, since $W(t)$ satisfies (3.24) and $R(t, s ; x, \xi)$ belongs to $\gamma^{d, s} S\left(e^{-\varepsilon_{0}(\langle x\rangle\langle\xi\rangle)^{1 /(d+s-1)}}, g_{0}\right)$, it follows from Proposition 2.6 that $W(t, x, \xi)$ also

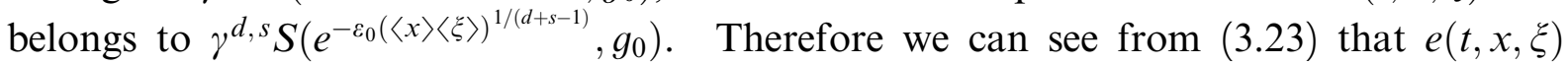
satisfies

$$
e(t, x, \xi)=\tilde{e}(t, 0 ; x, \xi)+\tilde{r}(t, x, \xi) \text {, }
$$

where $\tilde{r} \in \mathscr{C}^{1}\left([-T, T] ; \gamma^{d, s} S\left(e^{-\varepsilon_{0}(\langle x\rangle\langle\xi\rangle)^{1 /(d+s-1)}}, g_{0}\right)\right)$.

\section{Bicharacteristics.}

In this paragraph we will study some properties of the bicharacteristics of $a_{2}(x, \xi)$. Calculations are similar to those in $[\mathbf{1 8}, \S 2]$.

Let $(X(t ; y, \eta), \Xi(t ; y, \eta))$ be the solution of the Hamiltonian system (1.2).

LEMMA 4.1.

(1) If $x=X(t ; y, \eta)$ and $\xi=\Xi(t ; y, \eta)$, then $y=X(-t ; x, \xi)$ and $\eta=\Xi(-t ; x, \xi)$.

(2) If $x_{0}=X\left(t_{0} ; y, \eta\right)$ and $\xi_{0}=\Xi\left(t_{0} ; y, \eta\right)$, then $X\left(t+t_{0} ; y, \eta\right)=X\left(t ; x_{0}, \xi_{0}\right)$ and $\Xi\left(t+t_{0} ; y, \eta\right)=\Xi\left(t ; x_{0}, \xi_{0}\right)$.

These properties follow from the group-property of the solutions of ordinary differential equations.

Lemma 4.2. $X(t ; y, \eta)=X\left(\lambda t ; y, \lambda^{-1} \eta\right)$ and $\Xi(t ; y, \eta)=\lambda \Xi\left(\lambda t ; y, \lambda^{-1} \eta\right)$.

Proof. Set $X_{\lambda}(t):=X\left(\lambda t ; y, \lambda^{-1} \eta\right)$ and $\Xi_{\lambda}(t):=\lambda \Xi\left(\lambda t ; y, \lambda^{-1} \eta\right)$. Since the function $a_{2}(x, \xi)$ is 2-homogeneous, we have

$$
\begin{aligned}
& \dot{X}_{\lambda}(t)=\frac{d}{d t} X(\lambda t)=\lambda \partial_{\xi} a_{2}(X(\lambda t), \Xi(\lambda t))=\partial_{\xi} a_{2}(X(\lambda t), \lambda \Xi(\lambda t)) \\
& \dot{\Xi}_{\lambda}(t)=\frac{d}{d t}(\lambda \Xi)(\lambda t)=-\lambda^{2} \partial_{x} a_{2}(X(\lambda t), \Xi(\lambda t))=-\partial_{x} a_{2}(X(\lambda t), \lambda \Xi(\lambda t)) .
\end{aligned}
$$

$\left(X_{\lambda}(t), \Xi_{\lambda}(t)\right)$ is then the solution of the Hamiltonian system (1.2), with initial data $(y, \eta)$. By the unicity of the solution we have the result.

As in [18], we introduce the symbol $a(x, \xi)=\sqrt{2 a_{2}(x, \xi)}$. By Lemma 2.8, $a(x, \xi)$ belongs to $\gamma^{d, 1} S\left(|\xi|, g_{1}\right)$. Moreover there exists $C>0$ such that

$$
|a(x, \xi)| \geq C|\xi|
$$

Let us consider $\{x(t ; y, \eta), \eta(t ; y, \eta)\}$, the solution of the Hamiltonian system 


$$
\left\{\begin{array}{l}
\dot{x}_{j}(t)=\partial_{\xi_{j}} a(x(t ; y, \eta), \xi(t ; y, \eta)) \\
\dot{\xi}_{j}(t)=-\partial_{x_{j}} a(x(t ; y, \eta), \xi(t ; y, \eta)) \\
x_{j}(0)=y_{j} \\
\xi_{j}(0)=\eta_{j}
\end{array}\right.
$$

for $j=1, \ldots, n$. We can derive properties of $(X(t ; y, \eta), \Xi(t ; y, \eta))$ from that of $(x(t ; y, \eta), \xi(t ; y, \eta))$, since we have (cf. Lemma 2.3 in [18])

$$
(X(t ; y, \eta), \Xi(t ; y, \eta))=(x(a(y, \eta) t ; y, \eta), \xi(a(y, \eta) t ; y, \eta)) .
$$

Since $a(x, \xi) /|\xi|$ is bounded there exists globally in $t$ the solution of $(4.2)$, and consequently of (1.2).

By a similar computation as in Lemma 4.2, we can see that $x(t ; y, \eta)$ and $\eta(t ; y, \eta)$ are homogeneous in $\eta$ of degree zero and one respectively. Note also that we have

$$
a(x(t ; y, \eta), \xi(t ; y, \eta))=a(y, \eta), \quad \text { for all } t,
$$

since $(d / d t) a(x(t ; y, \eta), \xi(t ; y, \eta))=H_{a} a=0$.

Lemma 4.3. There exists $C>0$ such that

$$
C^{-1}|\eta| \leq|\xi(t ; y, \eta)| \leq C|\eta|
$$

for any $t \in \boldsymbol{R}$.

Proof. Let $C_{a}$ such that $C_{a}^{-1}|\xi| \leq|a(x, \xi)| \leq C_{a}|\xi|$, using (4.1) and (4.4), we have:

$$
\begin{aligned}
& |\xi(t ; y, \eta)| \geq C_{a}^{-1}|a(x(t ; y, \eta), \xi(t ; y, \eta))|=C_{a}^{-1}|a(y, \eta)| \geq C_{a}^{-2}|\eta| \\
& |\xi(t ; y, \eta)| \leq C_{a}|a(x(t ; y, \eta), \xi(t ; y, \eta))|=C_{a}|a(y, \eta)| \leq C_{a}^{2}|\eta| .
\end{aligned}
$$

Lemma 4.4. There exists $C>0$ such that

$$
C|\xi| \leq H_{a} \theta(x, \xi) \leq C^{-1}|\xi|
$$

Proof. The first inequality follows from the identity $H_{a_{2}}=a H_{a}$, inequality (4.5) and Assumption I-(4). The second follows from the fact that $a \in \gamma^{d, 1} S\left(|\xi|, g_{1}\right)$ and $\theta \in$ $\gamma^{d, 1} S\left(\langle x\rangle|\xi|, g_{1}\right)$.

Notations. In the following we note

$$
\Lambda=\left\{(t, y, \eta) \in \boldsymbol{R} \times \boldsymbol{R}^{n} \times \boldsymbol{R}^{n} \backslash\{0\} \mid \theta(y, \eta)=0\right\} .
$$

LEMMA 4.5. Let $\{x(t ; y, \eta), \eta(t ; y, \eta)\}$ be the solution of (4.2). Then there exists $C>0$ such that

$$
\begin{aligned}
& C^{-1}(|t|+\langle y\rangle) \leq\langle x(t ; y, \eta)\rangle \leq C(|t|+\langle y\rangle) \\
& C^{-1}|\eta| \leq \frac{1}{t} \theta(x(t ; y, \eta), \xi(t ; y, \eta)) \leq C|\eta|
\end{aligned}
$$

for $(t ; y, \eta) \in \Lambda$. 
Proof. We prove first (4.8). Integrating the identity $(d / d t) \theta(x(t), \xi(t))=H_{a} \theta(t)$, since $\theta(y, \eta)=0$, we have

$$
\theta(x(t), \xi(t))=\int_{0}^{t} H_{a} \theta(x(s), \xi(s)) d s
$$

and we obtain (4.8) using (4.5) and (4.6). We prove next (4.7). Since $a_{\xi}$ is bounded in $\boldsymbol{R}^{2 n}$, integrating the first equation of (4.2) we get the second inequality of (4.7). Since

$$
\theta(x(t), \xi(t)) \leq C\langle x(t)\rangle|\xi(t)|
$$

using (4.5) and (4.8) we obtain

$$
C^{-1}|t||\eta| \leq C\langle x(t)\rangle|\eta|
$$

then

$$
\langle x(t)\rangle \geq C^{\prime}|t| .
$$

Integrating the first equation of (4.2), since $\partial_{\xi_{j}} a$ is bounded, we get

$$
\langle x(t)\rangle \geq|y|-C^{\prime \prime}|t| \text {. }
$$

Combining (4.9) and (4.10) we get the first inequality of (4.7).

REMARK 4.6. By similar calculations, we can prove that

$$
\langle x(t ; y, \eta)\rangle \geq C(|t|+\langle y\rangle),
$$

for any $y, \eta \in \boldsymbol{R}^{n}$, so that using (4.3), we have

$$
\lim _{t \rightarrow+\infty}|X(t ; y, \eta)|=+\infty
$$

for any $y, \eta \in \boldsymbol{R}^{n}$.

We want to decompose $\Lambda$ into sets where we can estimate from below the derivatives of the function $\theta$. Let

$$
\begin{aligned}
& \Lambda_{i}=\left\{(t ; y, \eta) \in \Lambda|| \partial_{y_{j}} \theta(y, \eta)\left|\geq C^{*}\right| \eta \mid\right\} \\
& \Lambda_{i}^{\prime}=\left\{(t ; y, \eta) \in \Lambda|| \partial_{\eta_{j}} \theta(y, \eta)\left|\geq C^{*}\right| \eta \mid\right\} .
\end{aligned}
$$

We show that if $C^{*}$ is small enough, then

$$
\Lambda=\bigcup_{i=1}^{n} \Lambda_{i} \cup \bigcup_{i=1}^{n} \Lambda_{i}^{\prime}
$$

In fact, if

$$
\left|\partial_{y_{j}} \theta(y, \eta)\right| \leq C^{*}|\eta| \quad \text { and } \quad\left|\partial_{\eta_{j}} \theta(y, \eta)\right| \leq C^{*}|\eta|
$$

then $H_{a_{2}} \theta(y, \eta) \leq C_{a} C^{*}|\eta|^{2}$, where $C_{a}$ is some constant depending on the coefficients of $a_{2}$. Since, $H_{a_{2}} \theta \geq C|\eta|^{2}$, inequalities (4.11) cannot be verified if $C^{*}$ is too small.

In order to simplify the presentation, we will assume that

$$
\Lambda=\bigcup_{i=1}^{n} \Lambda_{i}
$$

We can treat the general case by similar computations. 
Notations. For $y \in \boldsymbol{R}^{n}$, we write $\tilde{y}=\left(y_{1}, \ldots, y_{i-1}, y_{i+1}, \ldots, y_{n}\right)$, and denote

$$
\begin{aligned}
\nabla_{t, \tilde{y}} x(t ; y, \eta) & =\left(\partial_{t} x, \partial_{y_{1}} x, \ldots, \partial_{y_{i-1}} x, \partial_{y_{i+1}} x, \ldots, \partial_{y_{n}} x\right) \\
\nabla_{\eta} x(t ; y, \eta) & =\left(\partial_{\eta_{1}} x, \ldots, \partial_{\eta_{n}} x\right) \\
\nabla x(t ; y, \eta) & =\left(\nabla_{t, \tilde{y}} x, \nabla_{\eta} x\right) \\
T(t ; y, \eta) & =\left(\begin{array}{c}
\nabla x(t ; y, \eta) \\
\nabla \xi(t ; y, \eta)
\end{array}\right) .
\end{aligned}
$$

$T(t)=T(t ; y, \eta)$ is a $2 n \times 2 n$ matrix. Differentiating (4.2) we get

$$
\frac{d}{d t} T(t)=A(t) T(t)
$$

where

$$
A(t)=\left(\begin{array}{cc}
a_{x \xi}(x(t), \xi(t)) & a_{\xi \xi}(x(t), \xi(t)) \\
-a_{x x}(x(t), \xi(t)) & -a_{x \xi}(x(t), \xi(t))
\end{array}\right) .
$$

By Hypothesis I-(3), (4.5) and (4.7) we have

$$
\begin{aligned}
& \left|a_{\xi \xi}(x(t), \xi(t))\right| \leq C|\eta|^{-1}, \\
& \left|a_{x \xi}(x(t), \xi(t))\right| \leq C(\langle y\rangle+|t|)^{-1-\delta}, \\
& \left|a_{x x}(x(t), \xi(t))\right| \leq C(\langle y\rangle+|t|)^{-2-\delta}|\eta|,
\end{aligned}
$$

for $(t, y, \eta) \in \Lambda$. We see that for $(t, y, \eta) \in \Lambda_{i}$

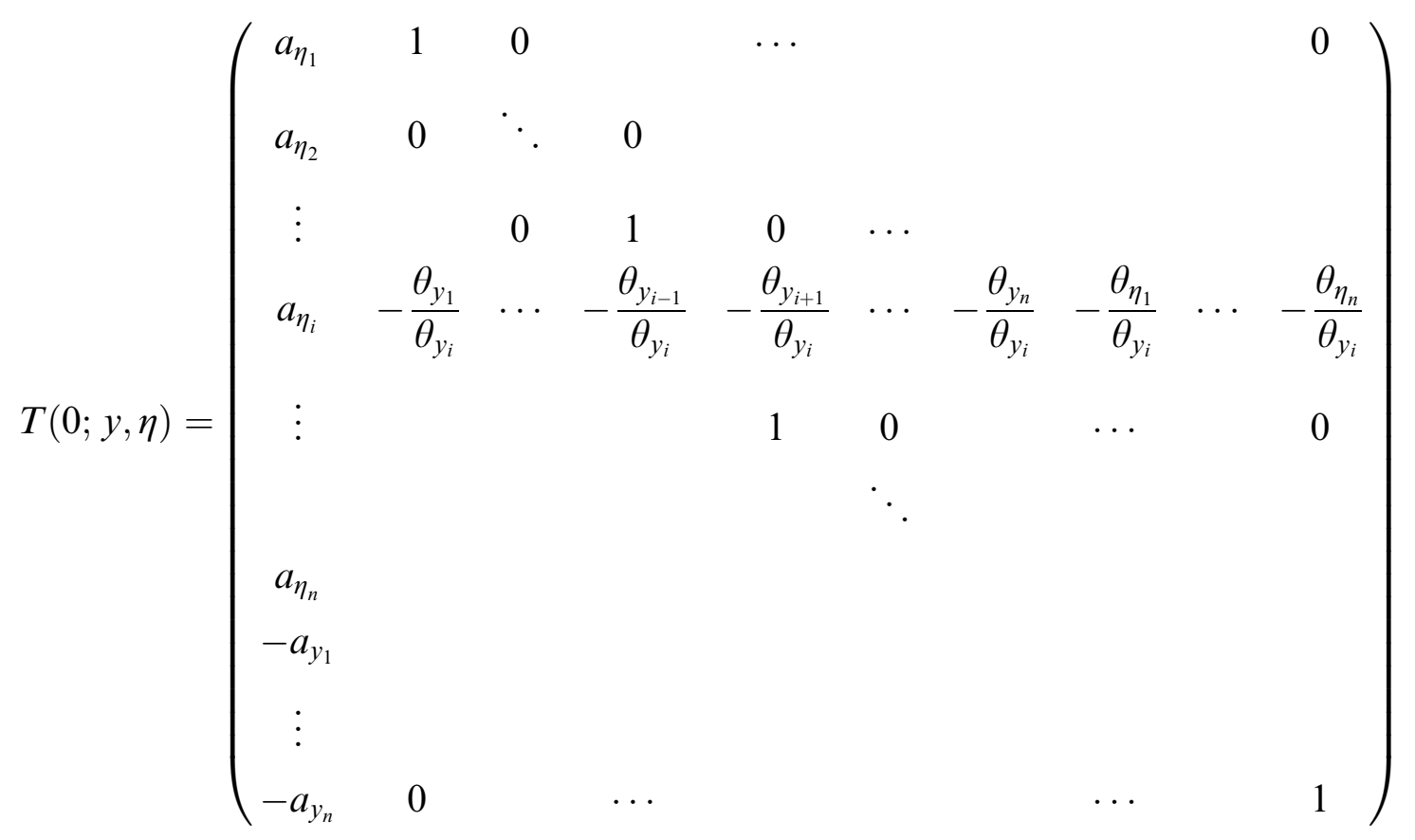

where $a_{y_{j}}=a_{y_{j}}(y, \eta), a_{\eta_{j}}=a_{\eta_{j}}(y, \eta), \theta_{y_{j}}=\theta_{y_{j}}(y, \eta), \theta_{\eta_{j}}=\theta_{\eta_{j}}(y, \eta)$. 
Proposition 4.7. Denote $T=\left[\begin{array}{ll}T_{11} & T_{12} \\ T_{21} & T_{22}\end{array}\right] . \quad T_{j k}$ are $\mathscr{C}^{\infty}\left(\Lambda_{i} ; \boldsymbol{R}^{n \times n}\right)$ and satisfy

$$
\begin{aligned}
& \left|\partial_{t}^{j} \partial_{\tilde{y}}^{\beta} \partial_{\eta}^{\alpha} T_{11}(t ; y, \eta)\right| \leq C j !|\alpha| !|\beta| !^{d} \rho^{-j-|\alpha|-|\beta|}(\langle y\rangle+|t|)^{1-j}\langle y\rangle^{-1-|\beta|}|\eta|^{-|\alpha|} \\
& \left|\partial_{t}^{j} \partial_{\tilde{y}}^{\beta} \partial_{\eta}^{\alpha} T_{21}(t ; y, \eta)\right| \leq C j !|\alpha| !|\beta| !^{d} \rho^{-j-|\alpha|-|\beta|}(\langle y\rangle+|t|)^{-j}\langle y\rangle^{-1-|\beta|}|\eta|^{1-|\alpha|} \\
& \left|\partial_{t}^{j} \partial_{\tilde{y}}^{\beta} \partial_{\eta}^{\alpha} T_{12}(t ; y, \eta)\right| \leq C j !|\alpha| !|\beta| !^{d} \rho^{-j-|\alpha|-|\beta|}(\langle y\rangle+|t|)^{1-j}\langle y\rangle^{-|\beta|}|\eta|^{-1-|\alpha|} \\
& \left|\partial_{t}^{j} \partial_{\tilde{y}}^{\beta} \partial_{\eta}^{\alpha} T_{22}(t ; y, \eta)\right| \leq C j !|\alpha| !|\beta| !^{d} \rho^{-j-|\alpha|-|\beta|}(\langle y\rangle+|t|)^{-j}\langle y\rangle^{-|\beta|}|\eta|^{-|\alpha|}
\end{aligned}
$$

for $(t, y, \eta) \in \Lambda_{i}$.

Proof. We recall first the following Lemma:

LeMma $4.8\left(\left[\mathbf{1 8}\right.\right.$, Lemma 2.4]). Let $w(t)=\left(\begin{array}{l}w_{1}(t) \\ w_{2}(t)\end{array}\right)$ be in $\mathscr{C}^{1}\left(\left[0,+\infty\left[; \boldsymbol{R}^{2 n}\right)\right.\right.$ and $F(t)=\left(\begin{array}{l}F_{1}(t) \\ F_{2}(t)\end{array}\right)$ in $\mathscr{C}^{0}\left(\left[0,+\infty\left[; \boldsymbol{R}^{2 n}\right)\right.\right.$ satisfying

$$
\frac{d}{d t} w(t)=A(t) w(t)+F(t)
$$

where $A(t)$ is defined in (4.13). Then there exists $C>0$ such that

$$
\begin{aligned}
\left|w_{1}(t)\right| \leq C & {\left[\left(1+|t|\langle y\rangle^{-1-\delta}\right)\left|w_{1}(0)\right|+|\eta|^{-1}\left|w_{2}(0)\right|(1+|t|)\right.} \\
& \left.+|t| \sup _{|s| \leq|t|}\left|F_{1}(s)\right|+|\eta|^{-1} \int_{0}^{t}\left|F_{2}(s)\right| d s\right] \\
\left|w_{2}(t)\right| \leq C & {\left[\langle y\rangle^{-1-\delta / 2}\left|w_{1}(0)\right||\eta|+\left|w_{2}(0)\right|+\sup _{|s| \leq|t|}\left|F_{1}(s)\right||\eta|+\int_{0}^{t}\left|F_{2}(s)\right| d s\right] }
\end{aligned}
$$

for $t \geq 0$ and $(y, \eta) \in \boldsymbol{R}^{2 n}$ with $\theta(y, \eta)=0$.

Using Lemma 4.8, we have

$$
\begin{aligned}
\left|\partial_{\tilde{y}_{k}} x(t ; y, \eta)\right| & \leq C\left(1+|t|\langle y\rangle^{-1-\delta}\right) \\
\left|\partial_{\tilde{y}_{k}} \xi(t ; y, \eta)\right| & \leq C|\eta|\langle y\rangle^{-1-\delta / 2} \\
\left|\partial_{\eta_{k}} x(t ; y, \eta)\right| & \leq C\left[\frac{|y|}{|\eta|}\left(1+|t|\langle y\rangle^{-1-\delta}\right)+(1+|t|)|\eta|^{-1}\right] \\
\left|\partial_{\eta_{k}} \xi(t ; y, \eta)\right| & \leq C
\end{aligned}
$$

for $(t ; y, \eta) \in \Lambda_{i}$. In fact, to prove (4.21) and (4.22), put $w_{1}(t)=\partial_{\tilde{y}_{k}} x(t)$ and $w_{2}(t)=\partial_{\tilde{y}_{k}} \xi(t)$. Then $w(t)$ satisfies (4.19) with $F(t)=0$. We have

$$
w_{1 j}(0)=\partial_{\tilde{y}_{k}} x_{j}(0)= \begin{cases}-\frac{\theta_{y_{k}}(y, \eta)}{\theta_{y_{i}}(y, \eta)} & \text { if } j=i \\ 1 & \text { if } j \neq i \text { and } k=j \\ 0 & \text { otherwise. }\end{cases}
$$


Then noting that $\left|\theta_{y_{i}}(y, \eta)\right| \geq C^{*}|\eta|$, if $(y, \eta) \in \Gamma_{i}$, we get $\left|w_{1 j}(0)\right| \leq C$. Since $w_{2}(0)=0$, we get (4.21) and (4.22) from (4.20). To prove (4.23) and (4.24), put $w_{1}(t)=\partial_{\eta_{k}} x(t)$ and $w_{2}(t)=\partial_{\eta_{k}} \xi(t)$, then $w(t)$ satisfies (4.19) with $F(t)=0$. Since, as above, $\left|w_{1}(0)\right| \leq C(|y| /|\eta|)$ and $\left|w_{2}(0)\right| \leq C$, we get (4.23) and (4.24) from (4.20). Since $\left|\partial_{t} x(t ; y, \eta)\right| \leq C$ and $\left|\partial_{t} \xi(t ; y, \eta)\right| \leq C|\eta| /\langle x(t)\rangle$, we obtain (4.15), (4.16), (4.17) and (4.18) for $j+|\alpha+\beta|=0$ from (4.21), (4.22), (4.23) and (4.24). We proceed then by induction on $j+|\alpha+\beta|$. Let $k \geq 1$, assuming (4.15) and (4.16) for $j+|\alpha+\beta| \leq k-1$, we prove (4.15) and (4.16) for $j+|\alpha+\beta|=k$. We consider first the case when $j=0 . \quad$ Let $w^{(\alpha, \beta)}=\left(\begin{array}{l}w_{1}^{(\alpha, \beta)} \\ w_{2}^{(\alpha, \beta)}\end{array}\right)=\left(\begin{array}{l}T_{11} \\ T_{21}\end{array}\right)$. We have

$$
\frac{d}{d t} w^{(\alpha, \beta)}(t)=A(t) w^{(\alpha, \beta)}(t)+F^{(\alpha, \beta)}(t)
$$

with $A$ given in (4.13) and $F^{(\alpha, \beta)}=\left(\begin{array}{l}F_{1}^{(\alpha, \beta)} \\ F_{2}^{(\alpha, \beta)}\end{array}\right)$ with

$$
\begin{aligned}
F_{j}^{(\alpha, \beta)}= & \sum_{\substack{\alpha^{\prime}+\alpha^{\prime \prime}=\alpha \\
\beta^{\prime}+\beta^{\prime \prime}=\beta \\
\alpha^{\prime \prime}+\beta^{\prime \prime}<\alpha+\beta}} \frac{\alpha !}{\alpha^{\prime} ! \alpha^{\prime \prime} !} \frac{\beta !}{\beta^{\prime} ! \beta^{\prime \prime} !} \partial_{\xi}^{\alpha^{\prime}} \partial_{x}^{\beta^{\prime}} A_{j 1}(t) w_{1}^{\left(\alpha^{\prime \prime}, \beta^{\prime \prime}\right)} \\
& +\sum_{\substack{\alpha^{\prime}+\alpha^{\prime \prime}=\alpha \\
\beta^{\prime}+\beta^{\prime \prime}=\beta \\
\alpha^{\prime \prime}+\beta^{\prime \prime}<\alpha+\beta}} \frac{\alpha !}{\alpha^{\prime} ! \alpha^{\prime \prime} !} \frac{\beta !}{\beta^{\prime} ! \beta^{\prime \prime} !} \partial_{\xi}^{\alpha^{\prime}} \partial_{x}^{\beta^{\prime}} A_{j 2}(t) w_{2}^{\left(\alpha^{\prime \prime}, \beta^{\prime \prime}\right)},
\end{aligned}
$$

for $j=1,2$, where $A(t)=A(x(t ; y, \eta), \xi(t ; y, \eta))$. In order to estimate $\partial_{\xi}^{\alpha^{\prime}} \partial_{x}^{\beta^{\prime}} A(t)$ we will use Lemma 2.8, with

$$
\begin{array}{lll}
f(z, \zeta)=A_{11}(t ; z, \zeta)=a_{x \xi}(x(t ; y, \eta), \xi(t ; y, \eta)), & m_{f}=C_{a}\langle z\rangle^{-1-\delta / 2}, \\
\varphi(y, \eta)=x(t ; y, \eta), & & m_{\varphi}=\langle y\rangle+|t|, \\
\psi(y, \eta)=\xi(t ; y, \eta), & m_{\psi}=C|\eta|,
\end{array}
$$

and noting that $m_{\varphi}(y, \eta) /\langle\varphi(y, \eta)\rangle$ and $m_{\psi}(y, \eta) /\langle\psi(y, \eta)\rangle$ are bounded, we obtain

$$
\begin{aligned}
\left|\partial_{t}^{j} \partial_{\eta}^{\alpha} \partial_{\tilde{y}}^{\beta} a_{x \xi}(x(t ; y, \eta), \xi(t ; y, \eta))\right| \leq & C_{0}(|\alpha|+1) !(|\beta|+1) !^{d} \rho_{0}^{-|\alpha+\beta|-2} \\
& (\langle y\rangle+|t|)^{-1-\delta / 2}\langle y\rangle^{-|\beta|-j}|\eta|^{-|\alpha|}
\end{aligned}
$$

for some $C_{0}$ and $\rho_{0}$ positif. By similar computations, we obtain

$$
\begin{aligned}
\left|\partial_{t}^{j} \partial_{\eta}^{\alpha} \partial_{\tilde{y}}^{\beta} a_{x x}(x(t ; y, \eta), \xi(t ; y, \eta))\right| \leq & C_{0}|\alpha| !(|\beta|+2) !^{d} \rho_{0}^{-|\alpha+\beta|-2} \\
& \times(\langle y\rangle+|t|)^{-2-\delta / 2}\langle y\rangle^{-|\beta|-j}|\eta|^{-|\alpha|+1} \\
\left|\partial_{t}^{j} \partial_{\eta}^{\alpha} \partial_{\tilde{y}}^{\beta} a_{\xi \xi}(x(t ; y, \eta), \xi(t ; y, \eta))\right| \leq & C_{0}(|\alpha|+2) !|\beta| !^{d} \rho_{0}^{-|\alpha+\beta|-2}\langle y\rangle^{-|\beta|-j}|\eta|^{-|\alpha|-1} .
\end{aligned}
$$


Then we have

$$
\begin{aligned}
\left|F_{1}^{(\alpha, \beta)}\right| \leq & C_{0} C \rho^{-|\alpha+\beta|} \rho_{0}^{-2}\langle y\rangle^{-1-|\beta|}|\eta|^{-|\alpha|}(\langle y\rangle+|t|)^{-\delta / 2} \\
& \times \sum_{\substack{\alpha^{\prime}+\alpha^{\prime \prime}=\alpha \\
\beta^{\prime}+\beta^{\prime \prime}=\beta \\
\alpha^{\prime \prime}+\beta^{\prime \prime}<\alpha+\beta}} \frac{\alpha !}{\alpha^{\prime} ! \alpha^{\prime \prime} !} \frac{\beta !}{\beta^{\prime} ! \beta^{\prime \prime} !}\left(\left|\alpha^{\prime}\right|+1\right) !\left|\alpha^{\prime \prime}\right| !\left(\left|\beta^{\prime}\right|+1\right) !^{d}\left|\beta^{\prime \prime}\right| !^{d}\left(\frac{\rho_{0}}{\rho}\right)^{-\left|\alpha^{\prime}+\beta^{\prime}\right|} \\
+ & C_{0} C \rho^{-|\alpha+\beta|} \rho_{0}^{-2}\langle y\rangle^{-1-|\beta|}|\eta|^{-|\alpha|} \\
& \times \sum_{\substack{\alpha^{\prime}+\alpha^{\prime \prime}=\alpha \\
\beta^{\prime}+\beta^{\prime \prime}=\beta \\
\alpha^{\prime \prime}+\beta^{\prime \prime}<\alpha+\beta}} \frac{\alpha !}{\alpha^{\prime} ! \alpha^{\prime \prime} !} \frac{\beta !}{\beta^{\prime} ! \beta^{\prime \prime} !}\left(\left|\alpha^{\prime}\right|+2\right) !\left|\alpha^{\prime \prime}\right| !\left|\beta^{\prime}\right| !^{d}\left|\beta^{\prime \prime}\right| !{ }^{d}\left(\frac{\rho_{0}}{\rho}\right)^{-\left|\alpha^{\prime}+\beta^{\prime}\right|} .
\end{aligned}
$$

Now $\sum_{\begin{array}{c}\alpha^{\prime}+\alpha^{\prime \prime}=\alpha \\ \beta^{\prime}+\beta^{\prime \prime}=\beta \\ \alpha^{\prime \prime}+\beta^{\prime \prime}<\alpha+\beta\end{array}} \cdots=\sum_{\begin{array}{c}\alpha^{\prime}+\alpha^{\prime \prime}=\alpha \\ \beta^{\prime}+\beta^{\prime \prime}=\beta \\ \alpha^{\prime}>0\end{array}} \cdots+\sum_{\begin{array}{c}\alpha^{\prime}+\alpha^{\prime \prime}=\alpha \\ \beta^{\prime}+\beta^{\prime \prime}=\beta \\ \beta^{\prime}>0\end{array}} \cdots$, so that, using Lemma 2.2 we obtain

$$
\left|F_{1}^{(\alpha, \beta)}\right| \leq \frac{C C^{\prime} \rho}{\left(\rho_{0}-\rho\right)\left(\rho_{0}-2^{d} \rho\right)}|\alpha| !|\beta| !^{d} \rho^{-|\alpha+\beta|}\langle y\rangle^{-1-|\beta|}|\eta|^{-|\alpha|}\left[(\langle y\rangle+|t|)^{-\delta / 2}+1\right],
$$

for some $C>0$. By similar computations

$$
\left|F_{2}^{(\alpha, \beta)}\right| \leq \frac{C C^{\prime} \rho}{\left(\rho_{0}-\rho\right)\left(\rho_{0}-2^{d} \rho\right)}|\alpha| !|\beta| !^{d} \rho^{-|\alpha+\beta|}\langle y\rangle^{-|\beta|}|\eta|^{-|\alpha|+1}(\langle y\rangle+|t|)^{-1-\delta / 2} .
$$

Applying Lemma 4.8, since $w^{(\alpha, \beta)}(0)=0$, we get

$$
\begin{aligned}
\left|w_{1}^{(\alpha, \beta)}(t)\right| \leq & |t| \frac{C_{0} C^{\prime \prime} \rho}{\left(\rho_{0}-\rho\right)\left(\rho_{0}-2^{d} \rho\right)}|\alpha| !|\beta| !^{d} \rho^{-|\alpha+\beta|} \\
& \times\left[\sup _{|s| \leq|t|}\left[(\langle y\rangle+|s|)^{-\delta / 2}+1\right]+\int_{0}^{t}(\langle y\rangle+|s|)^{-1-\delta / 2} d s\right]\langle y\rangle^{-|\beta|}|\eta|^{-|\alpha|} \\
\leq & \frac{C C^{\prime \prime \prime} \rho}{\left(\rho_{0}-\rho\right)\left(\rho_{0}-2^{d} \rho\right)}|\alpha| !|\beta| !^{d} \rho^{-|\alpha+\beta|}(\langle y\rangle+|t|)\langle y\rangle^{-|\beta|}|\eta|^{-|\alpha|} .
\end{aligned}
$$

Choosing $\rho$ small so that $C^{\prime \prime \prime} \rho /\left(\rho_{0}-\rho\right)\left(\rho_{0}-2^{d} \rho\right)<1$ we obtain (4.15). By a similar computation we can prove (4.16). To prove (4.15) and (4.16) for $j>0$ we remark that

$$
\begin{aligned}
& \partial_{t}^{j} \partial_{\eta}^{\alpha} \partial_{y}^{\beta} x(t ; y, \eta)=\partial_{t}^{j-1} \partial_{\eta}^{\alpha} \partial_{y}^{\beta} a_{\xi}(x(t ; y, \eta), \xi(t ; y, \eta)) \\
& \partial_{t}^{j} \partial_{\eta}^{\alpha} \partial_{y}^{\beta} \xi(t ; y, \eta)=-\partial_{t}^{j-1} \partial_{\eta}^{\alpha} \partial_{y}^{\beta} a_{x}(x(t ; y, \eta), \xi(t ; y, \eta))
\end{aligned}
$$

then the result follows from Lemma 2.8. By similar computations we can prove (4.17) and (4.18).

Using the Lemma 6.2 in the Appendix, we have

$$
\operatorname{det} T(0 ; y, \eta)=\frac{(-1)^{i-1}}{\theta_{y_{i}}(y, \eta)} H_{a} \theta(y, \eta) \text {. }
$$


Now $(d / d t) T(t)=A(t) T(t)$, implies $(d / d t) \operatorname{det} T(t)=\operatorname{trace} A(t) \operatorname{det} T(t)$ and since trace $A(t)=0$, we have

$$
\operatorname{det} T(t ; y, \eta)=\operatorname{det} T(0, y, \eta) \neq 0, \quad \text { for }(t ; y, \eta) \in \Lambda_{i} .
$$

$T(t ; y, \eta)$ is then invertible. We set

$$
S(t ; y, \eta)=T(t ; y, \eta)^{-1}=\left(\begin{array}{cc}
S_{11}(t) & S_{12}(t) \\
S_{21}(t) & S_{22}(t)
\end{array}\right) .
$$

Using again Lemma 6.2, we have

$$
S(0, y, \eta)=\left(\begin{array}{ll}
S_{11} & S_{12} \\
S_{21} & S_{22}
\end{array}\right)=\left[H_{a} \theta(y, \eta)\right]^{-1}\left(\begin{array}{cc}
\tilde{S}_{11} & \tilde{S}_{12} \\
\tilde{S}_{21} & \tilde{S}_{22}
\end{array}\right)
$$

where

$$
\begin{aligned}
& \tilde{S}_{11}=\left(\begin{array}{cccccc}
\theta_{y_{1}} & \theta_{y_{2}} & & \cdots & & \theta_{y_{n}} \\
b_{1} & -a_{\eta_{1}} \theta_{y_{2}} & & \cdots & & -a_{\eta_{1}} \theta_{y_{n}} \\
-a_{\eta_{2}} \theta_{y_{1}} & \ddots & & & & \\
\vdots & & b_{i-1} & -a_{\eta_{i-1}} \theta_{i} & \ldots & -a_{\eta_{i-1}} \theta_{y_{n}} \\
& & & -a_{\eta_{i-1}} \theta_{i} & b_{i+1} & \\
\vdots & & & & & \\
-a_{\eta_{n}} \theta_{y_{1}} & & \ldots & & -a_{\eta_{n}} \theta_{y_{n-1}} & b_{n}
\end{array}\right) \\
& \tilde{S}_{12}=\left(\begin{array}{ccc}
\theta_{\eta_{1}} & \cdots & \theta_{\eta_{n}} \\
-a_{\eta_{1}} \theta_{\eta_{1}} & \cdots & -a_{\eta_{1}} \theta_{\eta_{n}} \\
\vdots & & \vdots \\
-a_{\eta_{n}} \theta_{\eta_{1}} & \cdots & -a_{\eta_{n}} \theta_{\eta_{n}}
\end{array}\right) \\
& \tilde{S}_{21}=\left(\begin{array}{ccc}
-a_{y_{1}} \theta_{y_{1}} & \cdots & -a_{y_{1}} \theta_{y_{n}} \\
\vdots & & \vdots \\
-a_{y_{n}} \theta_{y_{1}} & \cdots & -a_{y_{n}} \theta_{y_{n}}
\end{array}\right) \\
& \tilde{S}_{22}=\left(\begin{array}{cccc}
c_{1} & -a_{y_{1}} \theta_{\eta_{2}} & \cdots & -a_{y_{1}} \theta_{\eta_{n}} \\
-a_{y_{2}} \theta_{\eta_{1}} & c_{2} & -a_{y_{2}} \theta_{\eta_{3}} & \cdots \\
& & \ddots & \\
\vdots & & & \\
-a_{y_{n}} \theta_{\eta_{1}} & \ldots & -a_{y_{n}} \theta_{\eta_{n-1}} & c_{n}
\end{array}\right) \text {, }
\end{aligned}
$$

where $b_{k}=H_{a} \theta(y, \eta)-a_{\eta_{k}}(y, \eta) \theta_{y_{k}}(y, \eta)$ and $c_{k}=H_{a} \theta(y, \eta)+a_{y_{k}}(y, \eta) \theta_{\eta_{k}}(y, \eta)$. We have

$$
\left|S_{11}\right| \leq C, \quad\left|S_{12}\right| \leq C|y||\eta|^{-1}, \quad\left|S_{21}\right| \leq C\langle y\rangle^{-1}, \quad\left|S_{22}\right| \leq C,
$$

for some constant $C$.

Differentiating the identity $T(t) S(t)=I$ with respect to $t$, we get 


$$
\frac{d}{d t} S(t)=-S(t) A(t)
$$

Put $u_{1}(t)=|\eta|\left|S_{12}(t)\right|$ and $u_{2}(t)=\left|S_{11}(t)\right|$. (4.27) gives

$$
\left\{\begin{array}{l}
S_{11}^{\prime}=-S_{11} A_{11}-S_{12} A_{21} \\
S_{12}^{\prime}=-S_{11} A_{12}-S_{12} A_{22}
\end{array}\right.
$$

then

$$
\left\{\begin{array}{l}
u_{1}^{\prime}(t) \leq\left|A_{22}\right| u_{1}(t)+|\eta|\left|A_{12}\right| u_{2}(t) \\
u_{2}^{\prime}(t) \leq|\eta|^{-1}\left|A_{21}\right| u_{1}(t)+\left|A_{11}\right| u_{2}(t)
\end{array}\right.
$$

Thanks to (4.14)

$$
\left\{\begin{array}{l}
u_{1}^{\prime}(t) \leq C(\langle y\rangle+|t|)^{-1-\delta} u_{1}(t)+C u_{2}(t) \\
u_{2}^{\prime}(t) \leq C(\langle y\rangle+|t|)^{-2-\delta} u_{1}(t)+C(\langle y\rangle+|t|)^{-1-\delta} u_{2}(t) .
\end{array}\right.
$$

We see that $u=\left(u_{1}, u_{2}\right)$ satisfies $[4.19)$ with $M=C, \rho_{1}(t)=C\left(t^{2}+\langle y\rangle^{2}\right)^{-(1+\delta) / 2}$, $\rho_{2}(t)=C\left(t^{2}+\langle y\rangle^{2}\right)^{-(1+\delta / 2) / 2}$, and $f_{1}=f_{2}=0$, then $u$ satisfies $(4.20)$, with $f_{1}=f_{2}=0$ :

$$
\begin{aligned}
& u_{1}(t) \leq C\left[\left(1+t\langle y\rangle^{-1-\delta / 2}\right) u_{1}(0)+(1+t) u_{2}(0)\right] \\
& u_{2}(t) \leq C\left[\langle y\rangle^{-1-\delta / 2} u_{1}(0)+u_{2}(0)\right]
\end{aligned}
$$

Now, using (4.26) we have $u_{1}(0) \leq C|y|, u_{2}(0) \leq C$, then (4.28) implies

$$
\begin{aligned}
& \left|S_{11}(t)\right| \leq C \\
& \left|S_{12}(t)\right| \leq C(|t|+\langle y\rangle)|\eta|^{-1},
\end{aligned}
$$

for $(t ; y, \eta) \in \Lambda_{i}$. Similarly, noting that $\left|S_{21}(0)\right| \leq C|\eta|\langle y\rangle^{-1}$ and $\left|S_{22}(0)\right| \leq C\langle y\rangle^{-1}$, we get

$$
\begin{aligned}
& \left|S_{21}(t)\right| \leq C|\eta|\langle y\rangle^{-1} \\
& \left|S_{22}(t)\right| \leq C(1+|t|\langle y\rangle),
\end{aligned}
$$

for $(t ; y, \eta) \in \Lambda_{i}$.

Proposition 4.9. There exist functions $t(x, \xi), y(x, \xi) \in \mathscr{C}^{\infty}\left(\boldsymbol{R}^{n} \times \boldsymbol{R}^{n} \backslash\{0\}\right)$ homogeneous in $\xi$ of degree 0 and $\eta(x, \xi) \in \mathscr{C}^{\infty}\left(\boldsymbol{R}^{n} \times \boldsymbol{R}^{n} \backslash\{0\}\right)$ homogeneous in $\xi$ of degree 1 such that $(t(x, \xi), y(x, \xi), \eta(x, \xi))$ belongs to $\Lambda$ and satisfy

$$
\begin{aligned}
& x(t(x, \xi), y(x, \xi), \eta(x, \xi))=x \\
& \xi(t(x, \xi), y(x, \xi), \eta(x, \xi))=\xi,
\end{aligned}
$$

for $(x, \xi) \in \boldsymbol{R}^{n} \times \boldsymbol{R}^{n} \backslash\{0\}$. Moreover

$$
\begin{aligned}
\left|\nabla_{x} t(x, \xi)\right|+ & \left|\nabla_{x} y(x, \xi)\right| \leq C \\
\left|\nabla_{\xi} t(x, \xi)\right|+\left|\nabla_{\xi} y(x, \xi)\right| & \leq C(|t(x, \xi)|+\langle y(x, \xi)\rangle)|\xi|^{-1} \\
\left|\nabla_{x} \eta(x, \xi)\right| & \leq C\langle y(x, \xi)\rangle^{-1}|\xi| \\
\left|\nabla_{\xi} \eta(x, \xi)\right| & \leq C\left(1+|t(x, \xi)|\langle y(x, \xi)\rangle^{-1}\right),
\end{aligned}
$$

for $(x, \xi) \in \boldsymbol{R}^{n} \times \boldsymbol{R}^{n} \backslash\{0\}$. 
Proof. Since the Jacobian $J(t ; y, \eta)=\operatorname{det} T(t ; y, \eta) \neq 0$ for $(t ; y, \eta) \in \Lambda_{i}$ and we have assumed (4.12), the local implicit function theorem of the mapping (4.31) holds evidently. We can prove the global version of implicit function theorem of the mapping (4.31) following the proof of Theorem 1.22 in [30]. To do so it suffices to show that for any compact set $K$ in $\boldsymbol{R}^{n} \times \boldsymbol{R}^{n} \backslash\{0\}$ the set

$$
\{(t ; y, \eta) \in \Lambda \mid(x(t ; y, \eta), \xi(t ; y, \eta))=(x, \xi) \text { for }(x, \xi) \in K\}
$$

is also compact in $\Lambda$. In fact, from Lemmas 4.4 and 4.5, we have

$$
\begin{aligned}
& C^{-1}|\xi| \leq|\eta| \leq C|\xi| \\
& |t| \leq C \frac{|\theta(x, \xi)|}{|\eta|} \leq C^{\prime}\langle x\rangle .
\end{aligned}
$$

Moreover the integration of (4.2) with respect to $t$ and (4.34) yield

$$
\langle y\rangle \leq\langle x\rangle+\int_{0}^{|t|}\left|\nabla_{\xi} a(x(s), \xi(s))\right| d s \leq C\langle x\rangle .
$$

Thus the inverse image of $K$ of the mapping $(x(t ; y, \eta), \xi(t ; y, \eta))=(x, \xi)$ is compact in A. Therefore (4.29) and (4.30) yield $\left|T(t ; y, \eta)^{-1}\right| \leq C$ if $(t ; y, \eta)$ varies in a compact set in $\Lambda_{i}$ and consequently we obtain the global implicit function theorem applying Theorem 1.22 in [30]. We next prove the estimates (4.32). Let $(t(x, \xi), y(x, \xi), \eta(x, \xi))$ be in $\Lambda_{i}$. We note that the local implicit function theorem implies that $(t(x, \xi), y(x, \xi)$, $\eta(x, \xi))$ are in $\mathscr{C}^{\infty}\left(\boldsymbol{R}^{n} \times \boldsymbol{R}^{n} \backslash\{0\}\right)$. Differentiating (4.31), we have

$$
\left(\begin{array}{ll}
\nabla_{x} t & \nabla_{\xi} t \\
\nabla_{x} \tilde{y} & \nabla_{\xi} \tilde{y} \\
\nabla_{x} \eta & \nabla_{\xi} \eta
\end{array}\right)=S(t(x, \xi), y(x, \xi), \eta(x, \xi)) .
$$

Using assumption (4.12), we obtain (4.32), by virtue of (4.29) and (4.30).

Lemma 4.10. $S_{j k} \in \mathscr{C}^{\infty}\left(\Lambda_{i} ; \boldsymbol{R}^{n \times n}\right)$ and

$$
\begin{aligned}
& \left|\partial_{t}^{j} \partial_{\tilde{y}}^{\beta} \partial_{\eta}^{\alpha} S_{11}(t ; y, \eta)\right| \leq C j !|\alpha| !|\beta| !^{d} \rho^{-j-|\alpha|-|\beta|}\langle y\rangle^{-|\beta|-j}|\eta|^{-|\alpha|} \\
& \left|\partial_{t}^{j} \partial_{\tilde{y}}^{\beta} \partial_{\eta}^{\alpha} S_{12}(t ; y, \eta)\right| \leq C j !|\alpha| !|\beta| !^{d} \rho^{-j-|\alpha|-|\beta|}(\langle y\rangle+|t|)\langle y\rangle^{-|\beta|-j}|\eta|^{-1-|\alpha|} \\
& \left|\partial_{t}^{j} \partial_{\tilde{y}}^{\beta} \partial_{\eta}^{\alpha} S_{21}(t ; y, \eta)\right| \leq C j !|\alpha| !|\beta| !^{d} \rho^{-j-|\alpha|-|\beta|}\langle y\rangle^{-|\beta|-j-1}|\eta|^{1-|\alpha|} \\
& \left|\partial_{t}^{j} \partial_{\tilde{y}}^{\beta} \partial_{\eta}^{\alpha} S_{22}(t ; y, \eta)\right| \leq C j !|\alpha| !|\beta| !^{d} \rho^{-j-|\alpha|-|\beta|}\left(1+|t|\langle y\rangle^{-1}\right)\langle y\rangle^{-|\beta|-j}|\eta|^{-|\alpha|}
\end{aligned}
$$

for $(t, y, \eta) \in \Lambda_{i}$.

Proof. Since $S(t)$ satisfies (4.27), (4.29) and (4.30), and $S(0)$ is given by (4.25), we can get the proof repeating the same arguments of Proposition 4.7.

Proposition 4.11. $t(x, \xi), y(x, \xi), \eta(x, \xi) \in \mathscr{C}^{\infty}\left(\boldsymbol{R}^{n} \times \boldsymbol{R}^{n} \backslash\{0\}\right)$ and 


$$
\begin{aligned}
\left|\partial_{x}^{\beta} \partial_{\xi}^{\alpha} t(x, \xi)\right|+\left|\partial_{x}^{\beta} \partial_{\xi}^{\alpha} y(x, \xi)\right| \leq & C|\alpha| !|\beta| !^{d} \rho^{-|\alpha|-|\beta|}\langle y(x, \xi)\rangle^{-|\beta|}|\xi|^{-|\alpha|} \\
& \times(|t(x, \xi)|+\langle y(x, \xi)\rangle)\left(1+|t(x, \xi)|\langle y(x, \xi)\rangle^{-1}\right)^{|\alpha+\beta|} \\
\left|\partial_{x}^{\beta} \partial_{\xi}^{\alpha} \eta(x, \xi)\right| \leq & C|\alpha| !|\beta| !^{d} \rho^{-|\alpha|-|\beta|}\langle y(x, \xi)\rangle^{-|\beta|}|\xi|^{1-|\alpha|} \\
& \times\left(1+|t(x, \xi)|\langle y(x, \xi)\rangle^{-1}\right)^{|\alpha+\beta|}
\end{aligned}
$$

for $x, \xi \in \boldsymbol{R}^{n}$.

Proof. By the local implicit function theorem we have that $t(x, \xi), y(x, \xi)$ and $\eta(x, \xi)$ belong to $\mathscr{C}^{\infty}\left(\boldsymbol{R}^{n} \times \boldsymbol{R}^{n} \backslash\{0\}\right)$.

Assume $(t(x, \xi), y(x, \xi), \eta(x, \xi)) \in \Lambda_{i}$, for some $i$. Since

$$
\left(\begin{array}{ll}
\nabla_{x} t & \nabla_{\xi} t \\
\nabla_{x} \tilde{y} & \nabla_{\xi} \tilde{y} \\
\nabla_{x} \eta & \nabla_{\xi} \eta
\end{array}\right)=S(t(x, \xi), y(x, \xi), \eta(x, \xi)),
$$

we see that (4.36) and (4.37) are proved for $(\alpha, \beta)=(0,0)$ in Proposition 4.9. Let $k \geq 1$, and assume (4.36) and (4.37)] are valid for $|\alpha+\beta| \leq k-1$. Noting that

$$
\begin{aligned}
\left(\nabla_{x} t, \nabla_{x} \tilde{y}\right) & =S_{11}(t(x, \xi), y(x, \xi), \eta(x, \xi)) \\
\left(\nabla_{\xi} t, \nabla_{\xi} \tilde{y}\right) & =S_{12}(t(x, \xi), y(x, \xi), \eta(x, \xi)) \\
\nabla_{x} \eta & =S_{21}(t(x, \xi), y(x, \xi), \eta(x, \xi)) \\
\nabla_{\xi} \eta & =S_{22}(t(x, \xi), y(x, \xi), \eta(x, \xi))
\end{aligned}
$$

to prove (4.36) and (4.37) it's enough to prove the following estimates

$$
\begin{aligned}
\left|\partial_{x}^{\beta} \partial_{\xi}^{\alpha} S_{11}(x, \xi)\right| \leq & C|\alpha| !|\beta| !^{d} \rho^{-|\alpha|-|\beta|}\langle y(x, \xi)\rangle^{-|\beta|}|\xi|^{-|\alpha|}\left(1+|t(x, \xi)|\langle y(x, \xi)\rangle^{-1}\right)^{|\alpha+\beta|} \\
\left|\partial_{x}^{\beta} \partial_{\xi}^{\alpha} S_{12}(x, \xi)\right| \leq & C|\alpha| !|\beta| !^{d} \rho^{-|\alpha|-|\beta|}\langle y(x, \xi)\rangle^{-|\beta|}|\xi|^{-1-|\alpha|} \\
& \times(|t(x, \xi)|+\langle y(x, \xi)\rangle)\left(1+|t(x, \xi)|\langle y(x, \xi)\rangle^{-1}\right)^{|\alpha+\beta|} \\
\left|\partial_{x}^{\beta} \partial_{\xi}^{\alpha} S_{21}(x, \xi)\right| \leq & C|\alpha| !|\beta|^{d} \rho^{-|\alpha|-|\beta|}\langle y(x, \xi)\rangle^{-1-|\beta|}|\xi|^{1-|\alpha|}\left(1+|t(x, \xi)|\langle y(x, \xi)\rangle^{-1}\right)^{|\alpha+\beta|} \\
\left|\partial_{x}^{\beta} \partial_{\xi}^{\alpha} S_{22}(x, \xi)\right| \leq & C|\alpha| !|\beta| !^{d} \rho^{-|\alpha|-|\beta|}\langle y(x, \xi)\rangle^{-|\beta|}|\xi|^{-|\alpha|}\left(1+|t(x, \xi)|\langle y(x, \xi)\rangle^{-1}\right)^{|\alpha+\beta|},
\end{aligned}
$$

where $(x, \xi)$ is the inverse image in $\Lambda_{i}$ of the mapping (4.31), and $S_{j l}(x, \xi)=S_{j l}(t(x, \xi)$, $y(x, \xi), \eta(x, \xi))$. The proof of these inequality follows from Lemma 2.8.

\section{Cut off function.}

For $\xi \in \boldsymbol{R}^{n} \backslash\{0\}$, we note $\tilde{\xi}=\xi /|\xi|$. Let

$$
\begin{aligned}
U_{\varepsilon}\left(y_{0}, \eta_{0}\right) & =\left\{(x, \xi)|| x-y_{0}|+| \tilde{\xi}-\tilde{\eta}_{0} \mid<\varepsilon_{0}\right\} \\
K_{\varepsilon}^{ \pm}\left(y_{0}, \eta_{0}\right) & =\left\{(x( \pm t, x, \xi), \xi( \pm t, x, \xi)) \mid t \geq 0,(x, \xi) \in U_{\varepsilon}\left(y_{0}, \eta_{0}\right)\right\} .
\end{aligned}
$$


LEMMA 5.1. $K_{\varepsilon}^{ \pm}\left(y_{0}, \eta_{0}\right)$ is a neighborhood of the bicharacteristic passing through $\left(y_{0}, \eta_{0}\right)$ and there exists $\varepsilon$ such that

$$
K_{\varepsilon}^{ \pm}\left(y_{0}, \eta_{0}\right) \subset \Gamma_{\varepsilon_{0}}^{ \pm}\left(y_{0}, \eta_{0}\right) \times\left\{\xi \in \boldsymbol{R}^{n}|| \tilde{\xi}-\tilde{\eta}_{0} \mid \leq \varepsilon_{0}\right\} .
$$

ProOF. We prove the Lemma only for $K_{\varepsilon}^{-}\left(y_{0}, \eta_{0}\right)$. The proof for $K_{\varepsilon}^{+}\left(y_{0}, \eta_{0}\right)$ is similar. We remark at first that, proceeding as in the proof of (4.21)-(4.24), we can show that

$$
\begin{array}{ll}
\left|\partial_{y_{k}} x(t ; y, \eta)\right| \leq C(1+|t|), & \left|\partial_{\eta_{k}} x(t ; y, \eta)\right| \leq C \frac{(1+|y|+|t|)}{|\eta|}, \\
\left|\partial_{y_{k}} \xi(t ; y, \eta)\right| \leq C|\eta|, & \left|\partial_{\eta_{k}} \xi(t ; y, \eta)\right| \leq C
\end{array}
$$

for $(y, \eta) \in \boldsymbol{R}^{n} \times \boldsymbol{R}^{n} \backslash\{0\}$. If $\left(x^{*}, \xi^{*}\right) \in K_{\varepsilon}^{-}\left(y_{0}, \eta_{0}\right)$, let $t_{0}, x_{0}, \xi_{0}$ such that $t_{0} \geq 0$, $\left|x_{0}-y_{0}\right|+\left|\tilde{\xi}_{0}-\tilde{\eta}_{0}\right|<\varepsilon_{0}$ and $x^{*}=x\left(-t_{0}, x_{0}, \xi_{0}\right), \xi^{*}=\xi\left(-t_{0}, x_{0}, \xi_{0}\right)$. Let

$$
\delta=\left|x_{0}-y_{0}\right|+\left|\tilde{\xi}_{0}-\tilde{\eta}_{0}\right| \text {. }
$$

Given $(x, \xi)$ near $\left(x^{*}, \xi^{*}\right)$, let $x_{1}=x\left(t_{0}, x, \xi\right), \xi_{1}=\xi\left(t_{0}, x, \xi\right)$. Using Lemma 4.1 and $(5.2)$, we have

$$
\begin{aligned}
\left|x_{1}-x_{0}\right| & =\left|x\left(t_{0}, x, \xi\right)-x\left(t_{0}, x^{*}, \xi^{*}\right)\right| \\
& \leq C\left(1+t_{0}\right)\left(\left|x-x^{*}\right|+\left|\tilde{\xi}-\tilde{\xi}^{*}\right|\right)<\frac{\varepsilon_{0}-\delta}{2},
\end{aligned}
$$

if $(x, \xi)$ is sufficiently close to $\left(x^{*}, \xi^{*}\right)$. By similar arguments, we get that if $(x, \xi)$ is sufficiently close to $\left(x^{*}, \xi^{*}\right)$, then

$$
\left|\tilde{\xi}_{1}-\tilde{\xi}_{0}\right|<\frac{\varepsilon_{0}-\delta}{2}
$$

Combining (5.4), (5.5) and (5.6) we have then

$$
\left|x_{1}-y_{0}\right|+\left|\tilde{\xi}_{1}-\tilde{\eta}_{0}\right| \leq\left|x_{1}-x_{0}\right|+\left|\tilde{\xi}_{1}-\tilde{\xi}_{0}\right|+\left|x_{0}-y_{0}\right|+\left|\tilde{\xi}_{0}-\tilde{\eta}_{0}\right|<\varepsilon_{0} .
$$

Using again Lemma 4.1, we get $x=x\left(-t_{0}, x_{1}, \xi_{1}\right)$ and $\xi=\xi\left(-t_{0}, x_{1}, \xi_{1}\right)$ then $(x, \xi) \in$ $K_{\varepsilon}^{-}\left(y_{0}, \eta_{0}\right)$.

Now we prove (5.1). Let $\left(x^{*}, \xi^{*}\right) \in K_{\varepsilon}^{-}\left(y_{0}, \eta_{0}\right)$, using (4.3), and proceeding as before,

$$
\begin{aligned}
\left|x^{*}-X\left(-\frac{t_{0}}{a\left(y_{0}, \eta_{0}\right)}, y_{0}, \eta_{0}\right)\right| & =\left|x\left(-t_{0}, x_{0}, \xi_{0}\right)-x\left(-t_{0}, y_{0}, \eta_{0}\right)\right| \\
& \leq C\left(1+t_{0}\right)\left(\left|x_{0}-y_{0}\right|+\left|\tilde{\xi}_{0}-\eta_{0}\right|\right) \\
& \leq C \varepsilon\left(1+t_{0}\right) .
\end{aligned}
$$

Similarly we have

$$
\left|\tilde{\xi}^{*}-\tilde{\eta}_{0}\right| \leq C \varepsilon .
$$

We can see from (5.7) and (5.8) that (5.1) holds true if we choose $\varepsilon$ small enough. 
LeMma 5.2. Let $a \in \mathscr{C}^{1}\left(\boldsymbol{R}^{n} \backslash\{0\}\right)$ a 1-homogeneous function such that a( $\left.\xi\right)>0$ for all $\xi \in \boldsymbol{R}^{n} \backslash\{0\}$. Then there exist $C_{1}, C_{2}>0$ such that

$$
C_{1}|\tilde{\xi}-\tilde{\eta}| \leq\left|\frac{\xi}{a(\xi)}-\frac{\eta}{a(\eta)}\right| \leq C_{2}|\tilde{\xi}-\tilde{\eta}|
$$

for all $\xi, \eta \in \boldsymbol{R}^{n} \backslash\{0\}$.

Proof. The first inequality follows from the identity

$$
\left|\frac{\xi}{a(\xi)}-\frac{\eta}{a(\eta)}\right|^{2}=\left[\frac{|\xi|}{a(\xi)}-\frac{|\eta|}{a(\eta)}\right]^{2}+\frac{|\xi|}{a(\xi)} \frac{|\eta|}{a(\eta)}|\tilde{\xi}-\tilde{\eta}|^{2},
$$

and $a(\xi) \leq C|\xi|$. For the second one, we have

$$
\begin{aligned}
\left|\frac{\xi}{a(\xi)}-\frac{\eta}{a(\eta)}\right| & =\left|\frac{\tilde{\xi}}{a(\tilde{\xi})}-\frac{\tilde{\eta}}{a(\tilde{\eta})}\right|=\left|\frac{a(\tilde{\eta}) \tilde{\xi}-a(\tilde{\xi}) \tilde{\eta}}{a(\tilde{\xi}) a(\tilde{\eta})}\right| \\
& \leq \frac{|a(\tilde{\eta}) \tilde{\xi}-a(\tilde{\xi}) \tilde{\xi}|+|a(\tilde{\xi}) \tilde{\xi}-a(\tilde{\xi}) \tilde{\eta}|}{a(\tilde{\xi}) a(\tilde{\eta})} \\
& \leq \frac{|a(\tilde{\eta})-a(\tilde{\xi})|}{a(\tilde{\xi}) a(\tilde{\eta})}+\frac{|\tilde{\xi}-\tilde{\eta}|}{a(\tilde{\xi}) a(\tilde{\eta})} \leq C|\tilde{\xi}-\tilde{\eta}|
\end{aligned}
$$

Let $\tilde{\theta}(x, \xi)=\theta(x, \xi) / a(x, \xi), \quad$ where $\quad a(x, \xi)=\sqrt{2 a_{2}(x, \xi)}$. Let $\left.\left.\quad s \in\right] 1, d\right] \quad$ and $\chi(t) \in C_{0}^{\infty}(\boldsymbol{R})$ such that $\chi(t)=1$ for $|t| \leq 1 / 2, \quad \chi(t)=0$ for $|t| \geq 1, \quad \chi(-t)=\chi(t)$, $t \chi^{\prime}(t) \leq 0$ and $0 \leq \chi(t) \leq 1$ and moreover

$$
\left|\partial_{t}^{k} \chi(t)\right| \leq A_{0}^{k+1} k !^{d}
$$

for $t \in \boldsymbol{R}$ and $k \in \boldsymbol{N}$.

Let

$$
\begin{aligned}
f_{1}(x, \xi) & =\chi\left(\frac{\left|x-y_{0}\right|}{\varepsilon_{1}}+\frac{1}{\varepsilon_{1}}\left|\frac{\xi}{a(x, \xi)}-\frac{\eta_{0}}{a\left(y_{0}, \eta_{0}\right)}\right|\right) \\
f_{2}(x, \xi) & =\chi\left(\frac{\tilde{\theta}(x, \xi)}{\varepsilon_{2}}\right) \frac{H_{a} \tilde{\theta}(x, \xi)}{M} \\
\psi_{1}^{ \pm}(x, \xi) & =\chi\left(\frac{\tilde{\theta}(x, \xi)}{\varepsilon_{3}\left\langle x-y_{0}\right\rangle}\right) \int_{0}^{\infty} f_{1}(x(\mp t, x, \xi), \xi(\mp t, x, \xi)) d t \\
\psi_{2}^{ \pm}(x, \xi) & =\left[1-\chi\left(\frac{\tilde{\theta}(x, \xi)}{\varepsilon_{3}\left\langle x-y_{0}\right\rangle}\right)\right] \int_{0}^{\infty} f_{2}(x(\mp t, x, \xi), \xi(\mp t, x, \xi)) d t,
\end{aligned}
$$

and

$$
\psi^{ \pm}(x, \xi)=\frac{1-\chi(h a(x, \xi))}{a(x, \xi)}\left[\psi_{1}^{ \pm}(x, \xi)+\psi_{2}^{ \pm}(x, \xi)\right] .
$$


Proposition 5.3. If $\varepsilon_{1}, \varepsilon_{2}$ and $\varepsilon_{3}$ are sufficiently small then

(1) $\psi^{ \pm}(x, \xi) \in \gamma^{d} S\left(1, g_{1}\right)$;

(2) $\psi^{ \pm}(x, \xi) \geq 0$ and $\psi^{ \pm}\left(y_{0}, \eta_{0}\right)>0$;

(3) $\operatorname{supp} \psi^{ \pm}(x, \xi) \subset K_{\varepsilon_{1}}^{ \pm}\left(y_{0}, \eta_{0}\right)$;

(4) $\operatorname{supp} H_{a_{2}} \psi^{ \pm}(x, \xi) \subset K_{\varepsilon_{1}}^{ \pm}\left(y_{0}, \eta_{0}\right)$ and $H_{a_{2}} \psi^{ \pm}(x, \xi) \leq 0$.

Proof. As before, we will prove the Proposition only for $\psi^{-}$, and we will note, for brevity $\psi, \psi_{1}, \psi_{2}, K_{\varepsilon_{1}}$ instead of $\psi^{-}, \psi_{1}^{-}, \psi_{2}^{-}, K_{\varepsilon_{1}}^{-}$.

1) Let us prove first the identity

$$
\int_{0}^{\infty} f(x(t, x, \xi), \xi(\tau, x, \xi)) d t=\int_{t(x, \xi)}^{\infty} f(x(\tau, y(x, \xi), \eta(x, \xi)), \xi(\tau, y(x, \xi), \eta(x, \xi))) d \tau .
$$

Using Lemma 4.1, we see that

$$
(x(\tau+t(x, \xi), y(x, \xi), \eta(x, \xi)), \xi(\tau+t(x, \xi), y(x, \xi), \eta(x, \xi)))
$$

is the solution at $t=\tau$, of the Hamiltonian system with initial data

$$
(x(t(x, \xi), y(x, \xi), \eta(x, \xi)), \xi(t(x, \xi), y(x, \xi), \eta(x, \xi)))=(x, \xi),
$$

so we obtain

$$
\begin{aligned}
& \int_{0}^{\infty} f(x(\tau, x, \xi), \xi(\tau, x, \xi)) d \tau \\
& \quad=\int_{0}^{\infty} f(x(\tau+t(x, \xi), y(x, \xi), \eta(x, \xi)), \xi(\tau+t(x, \xi), y(x, \xi), \eta(x, \xi))) d \tau
\end{aligned}
$$

Changing the variable in the last integral we obtain (5.10). From (4.7) and (4.31) we have

$$
|t(x, \xi)|+\langle y(x, \xi)\rangle \leq C\langle x\rangle .
$$

On the other hand, we have

$$
\langle x\rangle+|t(x, \xi)| \leq C\langle y(x, \xi)\rangle,
$$

for $(x, \xi) \in \operatorname{supp} \chi\left(\tilde{\theta}(x, \xi) / \varepsilon_{3}\left\langle x-y_{0}\right\rangle\right)$, if $\varepsilon_{3}$ is small enough. In fact, integrating the first equation of (4.2) with respect to $t$ we obtain

$$
y(x, \xi)=x-\int_{0}^{t(x, \xi)} a_{\xi}(x(s, y(x, \xi), \eta(x, \xi)), \xi(s, y(x, \xi), \eta(x, \xi))) d s,
$$

then $\langle y(x, \xi)\rangle \geq\langle x\rangle-C_{a}|t(x, \xi)|$, for some constant $C_{a}$ depending only on $a$. Now $|t(x, \xi)| \leq C|\tilde{\theta}(x, \xi)|$, and

$$
|\tilde{\theta}(x, \xi)| \leq \varepsilon_{3}\left\langle x-y_{0}\right\rangle \leq C_{y_{0}} \varepsilon_{3}\langle x\rangle
$$

for some constant $C_{y_{0}}$ depending only on $y_{0}$, if $(x, \xi) \in \operatorname{supp} \chi\left(\tilde{\theta}(x, \xi) / \varepsilon_{3}\left\langle x-y_{0}\right\rangle\right)$, then

$$
|t(x, \xi)| \leq C^{\prime} \varepsilon_{3}\langle x\rangle,
$$

if $(x, \xi) \in \operatorname{supp} \chi\left(\tilde{\theta}(x, \xi) / \varepsilon_{3}\left\langle x-y_{0}\right\rangle\right)$. If $\varepsilon_{3}$ is small enough, we have 


$$
\begin{aligned}
\langle x\rangle+|t(x, \xi)| & \leq\left(1+C^{\prime} \varepsilon_{3}\right)\langle x\rangle \leq \frac{1+C^{\prime} \varepsilon_{3}}{1-C_{a} C^{\prime} \varepsilon_{3}}\left(\langle x\rangle-C_{a} C^{\prime} \varepsilon_{3}\langle x\rangle\right) \\
& \leq \frac{1+C^{\prime} \varepsilon_{3}}{1-C_{a} C^{\prime} \varepsilon_{3}}\left(\langle x\rangle-C_{a}|t(x, \xi)|\right) \leq C^{\prime \prime}\langle y(x, \xi)\rangle .
\end{aligned}
$$

Putting (5.11), (5.12) and (5.13) in (4.36) and (4.37), we have

$$
\begin{aligned}
\left|\partial_{x}^{\beta} \partial_{\xi}^{\alpha} t(x, \xi)\right|+\left|\partial_{x}^{\beta} \partial_{\xi}^{\alpha} y(x, \xi)\right| & \leq C|\alpha| !|\beta| !^{d} \rho^{-|\alpha|-|\beta|}\langle x\rangle^{1-|\beta|}|\xi|^{-|\alpha|} \\
\left|\partial_{x}^{\beta} \partial_{\xi}^{\alpha} \eta(x, \xi)\right| & \leq C|\alpha| !|\beta| !^{d} \rho^{-|\alpha|-|\beta|}\langle x\rangle^{-|\beta|}|\xi|^{1-|\alpha|}
\end{aligned}
$$

for $(x, \xi) \in \operatorname{supp} \chi\left(\tilde{\theta}(x, \xi) / \varepsilon_{3}\left\langle x-y_{0}\right\rangle\right)$, and consequently we have from Proposition 4.7 and Lemma 2.8

$$
\begin{aligned}
& \left|\partial_{t}^{j} \partial_{x}^{\beta} \partial_{\xi}^{\alpha} x(s, y(x, \xi), \eta(x, \xi))\right| \leq C|\alpha| !^{d}(|\beta|+j) !^{d} \rho^{-|\alpha|-|\beta|-j}(\langle x\rangle+|t|)^{1-j}\langle x\rangle^{1-|\beta|}|\xi|^{-|\alpha|} \\
& \left|\partial_{t}^{j} \partial_{x}^{\beta} \partial_{\xi}^{\alpha} \xi(s, y(x, \xi), \eta(x, \xi))\right| \leq C|\alpha| !^{d}(|\beta|+j) !^{d} \rho^{-|\alpha|-|\beta|-j}(\langle x\rangle+|t|)^{1-j}\langle x\rangle^{-|\beta|}|\xi|^{1-|\alpha|}
\end{aligned}
$$

for $(x, \xi) \in \operatorname{supp} \chi\left(\tilde{\theta}(x, \xi) / \varepsilon_{3}\left\langle x-y_{0}\right\rangle\right)$. Thanks to (5.9), we can see that

$$
\left|\partial_{x}^{\beta} \partial_{\xi}^{\alpha} f_{1}(x, \xi)\right| \leq C|\alpha| !^{d}|\beta| !^{d} \rho^{-|\alpha|-|\beta|}\langle x\rangle^{-|\beta|}|\xi|^{-|\alpha|},
$$

hence, using Lemma 2.8, we have

$$
\left|\partial_{x}^{\beta} \partial_{\xi}^{\alpha} f_{1}(x(s, y(x, \xi), \eta(x, \xi)), \xi(s, y(x, \xi), \eta(x, \xi)))\right| \leq C|\alpha| !^{d}|\beta| !^{d} \rho^{-|\alpha|-|\beta|}\langle x\rangle^{-|\beta|}|\xi|^{-|\alpha|}
$$

for $(x, \xi) \in \operatorname{supp} \chi\left(\tilde{\theta}(x, \xi) / \varepsilon_{3}\left\langle x-y_{0}\right\rangle\right)$. Now, since

$$
\left|\partial_{x}^{\beta} \partial_{\xi}^{\alpha} \chi\left(\frac{\tilde{\theta}(x, \xi)}{\varepsilon_{3}\left\langle x-y_{0}\right\rangle}\right)\right| \leq C|\alpha| !^{d}|\beta| !^{d} \rho^{-|\alpha|-|\beta|}\langle x\rangle^{-|\beta|}|\xi|^{-|\alpha|}
$$

for $(x, \xi) \in \operatorname{supp} \chi\left(\tilde{\theta}(x, \xi) / \varepsilon_{3}\left\langle x-y_{0}\right\rangle\right)$, we obtain

$$
\left|\partial_{x}^{\beta} \partial_{\xi}^{\alpha} \psi_{1}(x, \xi)\right| \leq C|\alpha| !^{d}|\beta| !^{d} \rho^{-|\alpha|-|\beta|}\langle x\rangle^{-|\beta|}|\xi|^{-|\alpha|},
$$

for $(x, \xi) \in \boldsymbol{R}^{n} \times \boldsymbol{R}^{n} \backslash\{0\}$.

We can then see easily that

$$
\left|\partial_{x}^{\beta} \partial_{\xi}^{\alpha} \psi_{2}(x, \xi)\right| \leq C|\alpha| !^{d}|\beta| !^{d} \rho^{-|\alpha|-|\beta|}\langle x\rangle^{-|\beta|}|\xi|^{-|\alpha|},
$$

for $(x, \xi) \in \operatorname{supp}\left[1-\chi\left(\tilde{\theta}(x, \xi) / \varepsilon_{3}\left\langle x-y_{0}\right\rangle\right)\right]$ noting that

$$
\int_{0}^{\infty} \chi\left(\frac{\tilde{\theta}(x(t, x, \xi), \xi(t, x, \xi))}{\varepsilon_{2}}\right) H_{a} \tilde{\theta}(x(t, x, \xi), \xi(t, x, \xi)) d t=\int_{\tilde{\theta}(x, \xi)}^{\infty} \chi\left(\frac{t}{\varepsilon_{2}}\right) d t .
$$

Since $(1-\chi(h a(x, \xi))) / C a(x, \xi) \in \gamma^{d} S\left(1, g_{1}\right)$, we can see that $\psi(x, \xi) \in \gamma^{d} S\left(1, g_{1}\right)$.

2) It's obvious that $\psi(x, \xi) \geq 0$, for all $x, \xi \in \boldsymbol{R}^{n}$. Moreover $\psi_{2}\left(y_{0}, \eta_{0}\right)=0$, while

$$
\psi_{1}\left(y_{0}, \eta_{0}\right)=\int_{0}^{\infty} f_{1}\left(x\left(t, y_{0}, \eta_{0}\right), \xi\left(t, y_{0}, \eta_{0}\right)\right) d t
$$


and

$$
f_{1}\left(x\left(0, y_{0}, \eta_{0}\right), \xi\left(0, y_{0}, \eta_{0}\right)\right)=f_{1}\left(y_{0}, \eta_{0}\right)=1,
$$

and choosing $h$ suitably, we have $\psi\left(y_{0}, \eta_{0}\right)>0$.

3) Since $\operatorname{supp} f_{1}(x, \xi) \subset U_{\varepsilon}\left(y_{0}, \eta_{0}\right)$, we can see that

$$
\operatorname{supp} \int_{0}^{\infty} f_{1}(x(t, x, \xi), \xi(t, x, \xi)) d t \subset K_{\varepsilon_{1}}\left(y_{0}, \eta_{0}\right) .
$$

Moreover, since

$$
|\tilde{\theta}(x, \xi)| \leq C\left(\left|x-y_{0}\right|+\left|\tilde{\xi}-\tilde{\eta}_{0}\right|\right),
$$

for some positive constant $C$, choosing $\varepsilon_{2} \leq C \varepsilon_{1}$ we have

$$
\operatorname{supp} \int_{0}^{\infty} f_{2}(x(t, x, \xi), \xi(t, x, \xi)) d t \subset K_{\varepsilon_{1}}\left(y_{0}, \eta_{0}\right) .
$$

4) We have $H_{a_{2}}=a H_{a}$. Moreover (cf. [4, pp. 772-773])

$$
H_{a} \int_{0}^{+\infty} f(x(t, x, \xi), \xi(t, x, \xi)) d t=-f(x, \xi),
$$

then

$$
\begin{aligned}
H_{a} \psi(x, \xi)= & -f_{1}(x, \xi) \chi\left(\frac{\tilde{\theta}(x, \xi)}{\varepsilon_{3}\left\langle x-y_{0}\right\rangle}\right)+\int_{0}^{\infty} f_{1}(x(t), \xi(t)) d t H_{a} \chi\left(\frac{\tilde{\theta}(x, \xi)}{\varepsilon_{3}\left\langle x-y_{0}\right\rangle}\right) \\
& -f_{2}(x, \xi)\left[1-\chi\left(\frac{\tilde{\theta}(x, \xi)}{\varepsilon_{3}\left\langle x-y_{0}\right\rangle}\right)\right]-\int_{0}^{\infty} f_{2}(x(t), \xi(t)) d t H_{a} \chi\left(\frac{\tilde{\theta}(x, \xi)}{\varepsilon_{3}\left\langle x-y_{0}\right\rangle}\right),
\end{aligned}
$$

where, for the sake of brevity, we have set $x(t)=x(t, x, \xi)$ and $\xi(t)=\xi(t, x, \xi)$. As before, we can see that $\operatorname{supp} H_{a} \psi(x, \xi) \subset K_{\varepsilon_{1}}\left(y_{0}, \eta_{0}\right)$. Choosing $M$ so that $H_{a} \tilde{\theta}(x, \xi) \geq M$ and $\varepsilon_{2} \ll \varepsilon_{1}$, and using (5.14), we have $f_{2}(x, \xi) \geq f_{1}(x, \xi)$, so we only need to prove that

$$
H_{a} \chi\left(\frac{\tilde{\theta}(x, \xi)}{\varepsilon_{3}\left\langle x-y_{0}\right\rangle}\right)=\chi^{\prime}\left(\frac{\tilde{\theta}(x, \xi)}{\varepsilon_{3}\left\langle x-y_{0}\right\rangle}\right) H_{a}\left(\frac{\tilde{\theta}(x, \xi)}{\varepsilon_{3}\left\langle x-y_{0}\right\rangle}\right) \geq 0 .
$$

We prove at first that if $\varepsilon_{1} \ll \varepsilon_{3}$ we have

$$
\frac{\tilde{\theta}(x, \xi)}{\varepsilon_{3}\left\langle x-y_{0}\right\rangle} \leq \frac{1}{2}
$$

if $(x, \xi) \in K_{\varepsilon}^{ \pm}\left(y_{0}, \eta_{0}\right)$. Indeed, if $(x, \xi) \in K_{\varepsilon}^{ \pm}\left(y_{0}, \eta_{0}\right)$, let $t_{0} \geq 0, x_{0}$ and $\xi_{0}$ such that $t_{0} \geq 0, \quad x=x\left(-t_{0}, x_{0}, \xi_{0}\right), \quad \xi=\xi\left(-t_{0}, x_{0}, \xi_{0}\right) \quad$ and $\quad\left|x_{0}-y_{0}\right|+\left|\tilde{\xi}_{0}-\tilde{\eta}_{0}\right| \leq \varepsilon_{0} . \quad$ Using Lemma 4.1, we see that

$$
\begin{aligned}
\tilde{\theta}\left(x_{0}, \xi_{0}\right) & =\tilde{\theta}\left(x\left(t_{0}, x, \xi\right), \xi\left(t_{0}, x, \xi\right)\right) \\
& =\tilde{\theta}(x(0, x, \xi), \xi(0, x, \xi))+\int_{0}^{t_{0}} \frac{d}{d t} \tilde{\theta}(x(t, x, \xi), \xi(t, x, \xi)) d t \geq \tilde{\theta}(x, \xi),
\end{aligned}
$$


since

$$
\frac{d}{d t} \tilde{\theta}(x(t, x, \xi), \xi(t, x, \xi))=H_{a} \tilde{\theta}(x(t, x, \xi), \xi(t, x, \xi)) \geq 0
$$

Since $\tilde{\theta}(x, \xi) \in \gamma^{d, 1} S\left(\langle x\rangle, g_{1}\right)$, we get

$$
\begin{aligned}
\left|\frac{\tilde{\theta}\left(x_{0}, \xi_{0}\right)}{\varepsilon_{3}\left\langle x-y_{0}\right\rangle}\right| & \leq \frac{\left|\tilde{\theta}_{x}\right|\left|x_{0}-y_{0}\right|+\left|\tilde{\theta}_{\xi}\right|\left|\tilde{\xi}_{0}-\tilde{\eta}_{0}\right|}{\varepsilon_{3}\left\langle x-y_{0}\right\rangle} \\
& \leq C \frac{\left|x-y_{0}\right|+\left\langle x_{0}-y_{0}\right\rangle\left|\tilde{\xi}-\tilde{\eta}_{0}\right|}{\varepsilon_{3}\left\langle x-y_{0}\right\rangle} \leq \frac{C^{\prime} \varepsilon_{1}}{\varepsilon_{3}} \leq \frac{1}{2},
\end{aligned}
$$

if we take $\varepsilon_{1} \ll \varepsilon_{3}$. Now

$$
\begin{aligned}
H_{a}\left(\frac{\tilde{\theta}(x, \xi)}{\left\langle x-y_{0}\right\rangle}\right) & =\frac{H_{a} \tilde{\theta}(x, \xi)}{\left\langle x-y_{0}\right\rangle}+\tilde{\theta}(x, \xi) H_{a}\left\langle x-y_{0}\right\rangle^{-1} \\
& \geq \frac{C}{\left\langle x-y_{0}\right\rangle}-C^{\prime} \frac{\tilde{\theta}(x, \xi)}{\left\langle x-y_{0}\right\rangle^{2}}
\end{aligned}
$$

since $H_{a}\left\langle x-y_{0}\right\rangle^{-1} \leq C\left\langle x-y_{0}\right\rangle^{-2}$. Now, using (5.15),

$$
H_{a}\left(\frac{\tilde{\theta}(x, \xi)}{\left\langle x-y_{0}\right\rangle}\right) \geq \frac{\left(C-C^{\prime} \varepsilon_{3} / 2\right)}{\left\langle x-y_{0}\right\rangle} \geq 0,
$$

if we choose $\varepsilon_{3}$ small.

\section{Proof of the Theorem.}

The Cauchy problem (0.1) can be rewritten in the following form

$$
\begin{cases}\partial_{t} u(t, x)=-i a_{2}(x, D) w(t, x)+b(t, x, D) u(t, x), & t \in[-T, T], x \in \boldsymbol{R}^{n}, \\ u(0, x)=u_{0}(x), & x \in \boldsymbol{R}^{n},\end{cases}
$$

where

$$
\begin{aligned}
a(x, \xi) & =\sum_{j, k=1}^{n} a_{j k}(x) \xi_{j} \xi_{k} \\
b(t, x, \xi) & =\sum_{j, k=1}^{n} \partial_{x_{j}} a_{j k}(x) \xi_{k}+\sum_{j=1}^{n} b_{j}(t, x) \xi_{j}+b_{0}(t, x) .
\end{aligned}
$$

Let $\psi(x, \xi)$ the phase function constructed in paragraph 5, and

$$
p(x, \xi)=e(x, \xi)^{\kappa} \psi(x, \xi),
$$

where $e(x, \xi)=\left(1+a_{2}(x, \xi)\right)^{1 / 2}$. For $\rho>0$, denote by $E_{\rho}(t)$ the fundamental solution of the equation (3.3), given by Proposition 3.1. Put $v(t, x)=E_{\rho}(t) u(t, x)$, then

$$
\begin{aligned}
\partial_{t} v(t, x) & =\partial_{t} E_{\rho}(t) u(t, x)+E_{\rho}(t) \partial_{t} u(t, x) \\
& =\rho p(x, D) E_{\rho}(t) u(t, x)-i E_{\rho}(t) a_{2}(x, D) u(t, x)+E_{\rho}(t) b(t, x, D) u(t, x) \\
& =\left[\rho p(x, D)-i E_{\rho}(t) a_{2}(x, D) E_{\rho}(t)^{-1}+E_{\rho}(t) b(t, x, D) E_{\rho}(t)^{-1}\right] v(t, x) .
\end{aligned}
$$


Since $E_{\rho}(t)^{-1}=E_{\rho}(-t)$, using (3.6), we obtain

$$
\partial_{t} v(t, x)=-i a_{2}(x, D) v(t, x)+[\rho p(x, D)-\tilde{a}(t, x, D)+\tilde{b}(t, x, D)] v(t, x),
$$

where

$$
\begin{aligned}
& \tilde{a}(t, x, D)=i \rho \int_{0}^{t} E_{\rho}(s)[p, a] E_{\rho}(-s) d s \\
& \tilde{b}(t, x, D)=E_{\rho}(t) b(t, x, D) E_{\rho}(-t) .
\end{aligned}
$$

Let $\vartheta_{1}(t, x, \xi)$ be the phase function defined in (2.14). We claim that there exists $M$ such that

$$
\vartheta_{1}(t, x, \xi) \leq M \text { for all } t \in[0, T] \text { and }(x, \xi) \in K_{\varepsilon}^{-}\left(y_{0}, \eta_{0}\right) .
$$

Let $c(x, \xi)$ the symbol given in Proposition 2.16, and $C$ such that $|c(x, \xi)| \leq C$, choose $M$ such that

$$
M=\sup _{(x, \xi) \in U_{\varepsilon}\left(y_{0}, \eta_{0}\right)}\left[C|\theta(x, \xi)|+T e^{\kappa}(x, \xi)\right] .
$$

For $(x, \xi) \in K_{\varepsilon}^{-}\left(y_{0}, \eta_{0}\right)$, let $t_{0} \geq 0$ and $\left(x_{0}, \xi_{0}\right) \in U_{\varepsilon}\left(y_{0}, \eta_{0}\right)$ such that $x=x\left(-t_{0}, x_{0}, \xi_{0}\right)$ and $\xi=\xi\left(-t_{0}, x_{0}, \xi_{0}\right)$. If $\vartheta_{0}(x, \xi) \leq 0$, then

$$
\vartheta_{1}(t, x, \xi) \leq t e^{\kappa}(x, \xi) \leq T e^{\kappa}\left(x_{0}, \xi_{0}\right) \leq M .
$$

Assume now that $\vartheta_{0}(x, \xi)>0$, then from (2.15), we have that $\vartheta_{0}(x, \xi) \leq C \theta(x, \xi)$. Consider

$$
f(t)=C \theta\left(x\left(t, x_{0}, \xi_{0}\right), \xi\left(t, x_{0}, \xi_{0}\right)\right)+T e^{\kappa}\left(x\left(t, x_{0}, \xi_{0}\right), \xi\left(t, x_{0}, \xi_{0}\right)\right) .
$$

We have

$$
f^{\prime}(t)=\left.C H_{a} \theta(x, \xi)\right|_{\substack{x=x\left(t, x_{0}, \xi_{0}\right) \\ \xi=\xi\left(t, x_{0}, \xi_{0}\right)}}+\left.T H_{a} e^{\kappa}(x, \xi)\right|_{\substack{x=x\left(t, x_{0}, \xi_{0}\right) \\ \xi=\xi\left(t, x_{0}, \xi_{0}\right)}}>0,
$$

then $f(-t) \leq f(0)$, for all $t \geq 0$. In particular, for $t=t_{0}$,

$$
\begin{aligned}
\vartheta_{1}(t, x, \xi) & \leq C \theta(x, \xi)+T e^{\kappa}(x, \xi) \\
& =f\left(-t_{0}\right) \\
& \leq f(0) \\
& =C \theta\left(x_{0}, \xi_{0}\right)+T e^{\kappa}\left(x_{0}, \xi_{0}\right) \\
& \leq M
\end{aligned}
$$

We return to (6.2). Once more we change the unknown function. Let

$$
\vartheta(t, x, \xi)=\left[\vartheta_{1}(t, x, \xi)-M\right] \psi(x, \xi),
$$

and consider

$$
\varphi(t)=\varphi(t, x, \xi)=x \cdot \xi+i \varepsilon \vartheta(t, x, \xi) .
$$


We assume that $\varepsilon>0$ is chosen sufficiently small so that the operator $I_{\varphi(t)}(x, D)$ is invertible. Let $w(t, x)=I_{\varphi(t)}(x, D) v(t, x)$, we have

$$
\begin{aligned}
\partial_{t} w(t, x) & =\partial_{t} I_{\varphi(t)}(x, D) v(t, x)+I_{\varphi(t)}(x, D) \partial_{t} v(t, x) \\
& =\left[\partial_{t} I_{\varphi(t)} I_{\varphi(t)}^{-1}+I_{\varphi(t)}\left[-i a_{2}(x, D)+\rho p(x, D)-\tilde{a}(t, x, D)+\tilde{b}(t, x, D)\right] I_{\varphi(t)}^{-1}\right] w(t, x) .
\end{aligned}
$$

We get then the following Cauchy problem for $w$ :

$$
\left\{\begin{array}{l}
\partial_{t} w(t, x)=-i a_{2}(x, D) w(t, x)+\tilde{\tilde{b}}(t, x, D) w(t, x) \\
w(0, x)=I_{\varphi(0)}(x, D) u_{0},
\end{array}\right.
$$

where

$$
\begin{aligned}
\tilde{\tilde{b}}(t, x, \xi)= & \partial_{t} I_{\varphi(t)} I_{\varphi(t)}^{-1}-i\left[I_{\varphi(t)} a_{2}(x, \xi) I_{\varphi(t)}^{-1}-a_{2}(x, \xi)\right] \\
& +\rho I_{\varphi(t)} p(x, \xi) I_{\varphi(t)}^{-1}-I_{\varphi(t)} \tilde{a}(t, x, \xi) I_{\varphi(t)}^{-1}+I_{\varphi(t)} \tilde{b}(t, x, \xi) I_{\varphi(t)}^{-1}
\end{aligned}
$$

Now $\partial_{t} I_{\varphi(t)}=-\varepsilon \partial_{t} \vartheta_{\varphi(t)}$, and by (2.11) and (2.6),

$$
\begin{aligned}
\sigma\left(\partial_{t} I_{\varphi(t)} I_{\varphi(t)}^{-1}\right)(x, \xi) & =-\varepsilon \frac{\partial \vartheta}{\partial t}(t, x, \Phi(t, x, \xi))+\theta_{1}(t, x, \xi)+r_{1}(t, x, \xi) \\
& =-\varepsilon \frac{\partial \vartheta}{\partial t}(t, x, \xi)+\theta_{2}(t, x, \xi)+r_{1}^{\prime}(t, x, \xi)
\end{aligned}
$$

where $\theta_{1}, \theta_{2} \in \mathscr{C}^{0}\left([-T, T] ; \gamma^{d} S\left(1, g_{1}\right)\right.$ and $r_{1}, r_{1}^{\prime} \in \mathscr{C}^{0}\left([-T, T] ; \mathscr{A}_{-\varepsilon_{0},-\varepsilon_{0}}^{\kappa}\right)$. From $(2.9)$, we have

$$
\begin{aligned}
\sigma\left(I_{\varphi(t)} a_{2}(x, D) I_{\varphi(t)}^{-1}\right) & =a_{2}\left(x+i \varepsilon \nabla_{\xi} \vartheta(x, \Phi), \xi-i \varepsilon \nabla_{x} \vartheta(x, \Phi)\right)+a_{1}(x, \xi)+r_{2}(x, \xi) \\
& =a_{2}\left(x+i \varepsilon \nabla_{\xi} \vartheta(x, \xi), \xi-i \varepsilon \nabla_{x} \vartheta(x, \xi)\right)+a_{1}^{\prime}(x, \xi)+r_{2}^{\prime}(x, \xi) \\
& =a_{2}(x, \xi)-i \varepsilon H_{a_{2}} \vartheta(x, \xi)+\varepsilon^{2} c(x, \xi)+a_{1}^{\prime \prime}(x, \xi)+r_{2}^{\prime \prime}(x, \xi),
\end{aligned}
$$

where $a_{1}, a_{1}^{\prime}, a_{1}^{\prime \prime} \in \gamma^{d} S\left(\langle x\rangle^{-1}\langle\xi\rangle, g_{1}\right), \quad c \in \gamma^{d} S\left(\langle x\rangle^{2 \sigma-2}\langle\xi\rangle^{2 \delta}, g_{1}\right), \quad$ with $\operatorname{supp} c(x, \xi) \subset$ $\operatorname{supp} \psi(x, \xi)$ and $r_{2}, r_{2}^{\prime}, r_{2}^{\prime \prime} \in \mathscr{A}_{-\varepsilon_{0},-\varepsilon_{0}}^{\kappa}$, and also from (2.9)

$$
\sigma\left(I_{\varphi(t)} p(x, D) I_{\varphi(t)}^{-1}\right)=p(x, \xi)+p_{1}(x, \xi)+r_{3}(x, \xi),
$$

where $p_{1} \in \gamma^{d} S(1, g), r_{3} \in \mathscr{A}_{-\varepsilon_{0},-\varepsilon_{0}}^{\kappa}$. Similarly from (2.9)

$$
\begin{aligned}
\sigma\left(I_{\varphi(t)} \tilde{a}(t, x, D) I_{\varphi(t)}^{-1}\right) & =\tilde{a}(t, x, \xi)+\tilde{a}_{1}(t, x, \xi)+r_{4}(t, x, \xi) \\
& =H_{a_{2}} p(x, \xi)+\tilde{a}_{1}(t, x, \xi)+r_{4}(t, x, \xi)
\end{aligned}
$$

where $\tilde{a}_{1} \in \mathscr{C}^{0}\left([-T, T] ; \gamma^{d} S\left(1, g_{1}\right)\right)$ and $r_{4} \in \mathscr{C}^{0}\left([-T, T] ; \mathscr{A}_{-\varepsilon_{0},-\varepsilon_{0}}^{\kappa}\right)$, and making again use of (2.9)

$$
\sigma\left(I_{\varphi(t)} \tilde{b}(t, x, D) I_{\varphi(t)}^{-1}\right)=\tilde{b}(t, x, \xi)+\tilde{b}_{1}(t, x, \xi)+r_{5}(t, x, \xi),
$$

here $\tilde{b}_{1} \in \mathscr{C}^{0}\left([-T, T] ; \gamma^{d} S\left(1, g_{1}\right)\right)$ and $r_{5} \in \mathscr{C}^{0}\left([-T, T] ; \mathscr{A}_{-\varepsilon_{0},-\varepsilon_{0}}^{\kappa}\right)$. We get then

$$
\tilde{\tilde{b}}(t, x, \xi)=\tilde{\tilde{b}}_{1}(t, x, \xi)+\tilde{\tilde{b}}_{0}(t, x, \xi)+\tilde{\tilde{r}}(t, x, \xi),
$$


where

$$
\begin{gathered}
\tilde{\tilde{b}}_{1}(t, x, \xi)=\rho p(x, \xi)-\varepsilon\left(\partial_{t} \vartheta(t, x, \xi)+H_{a_{2}} \vartheta(t, x, \xi)\right)+H_{a_{2}} p(x, \xi)+\tilde{b}(t, x, \xi)+\varepsilon^{2} c(x, \xi) \\
\tilde{\tilde{b}}_{0} \in \mathscr{C}^{0}\left([-T, T] ; \gamma^{d} S\left(1, g_{1}\right)\right) \text { and } \tilde{\tilde{r}} \in \mathscr{C}^{0}\left([-T, T] ; \mathscr{A}_{-\varepsilon_{0},-\varepsilon_{0}}^{\kappa}\right) \text {. Noting that } \\
H_{a_{2}} \vartheta(t, x, \xi)=H_{a_{2}} \vartheta_{1}(t, x, \xi) \psi(x, \xi)+\left[\vartheta_{1}(t, x, \xi)-M\right] H_{a_{2}} \psi(x, \xi) \\
\geq H_{a_{2}} \vartheta_{1}(t, x, \xi) \psi(x, \xi)
\end{gathered}
$$

and

$$
H_{a_{2}} p(x, \xi)=e^{\kappa}(x, \xi) H_{a_{2}} \psi(x, \xi) \leq 0,
$$

we see then by use of (2.16) that we can choose $\rho$ and $\varepsilon$ suitably so that

$$
\operatorname{Re} \tilde{\tilde{b}}_{1}(t, x, \xi) \leq 0,
$$

for $x, \xi \in \boldsymbol{R}^{n},|x|,|\xi| \geq R$, if we choose $R>0$ sufficiently large. Using Proposition 2.17, we can see that the Cauchy problem (6.6) for $w$ has a solution belonging to $\mathscr{C}^{1}\left([0, T] ; L^{2}\right)$ since the initial data $w(0)=I_{\varphi(0)}(x, D) u_{0}(x)$ is in $L^{2}\left(\boldsymbol{R}^{n}\right)$ from the assumption of Theorem, and the choice of $\varphi(t, x, \xi)$ and $\vartheta(t, x, \xi)$ in (6.4) and (6.5). We obtain then the solution of the Cauchy problem (0.1) by

$$
u(t, x)=E_{\rho}(-t) I_{\varphi(t)}(x, D)^{-1} w(t, x) .
$$

In order to prove that $\left(y_{0}, \eta_{0}\right) \notin \mathrm{WF}_{1 / \kappa} u(t, \cdot)$, we prove that $\chi_{y}(x) \chi_{\eta}(D) u(t, x)$ belongs to $H_{\rho, \delta}^{\kappa}$, for some $\rho=\rho(t)$ positive and $\delta=\delta(t)$. It follows from (3.9) and (3.28) that we can write

$$
E_{\rho}(-t)=I_{\psi(-t)}(x, D)(\tilde{e}(-t, 0 ; x, D)+\tilde{r}(-t, x, D))
$$

where $\psi(t, x, \xi)=x \xi-i \rho \theta(t, x, \xi)$, with $\theta(t, x, \xi) \in \mathscr{C}^{1}\left([-T, T] ; \gamma^{d, 1} S\left(\langle\xi\rangle^{\kappa}, g_{1}\right)\right)$ the phase function constructed in Proposition 3.1 with $p(x, \xi)=e^{\kappa}(x, \xi) \chi_{y_{0}}(x) \chi_{\eta_{0}}(\xi), \tilde{e}(t, s ; x, \xi) \in$ $\mathscr{C}^{1}\left([-T, T]^{2} ; \gamma^{d}\left(1, g_{1}\right)\right)$ and $\tilde{r} \in \mathscr{C}^{1}\left([-T, T] ; \gamma^{d} S\left(e^{-\varepsilon_{0}(\langle x\rangle\langle\xi\rangle)^{1 /(d+s-1)}}, g_{0}\right)\right)$. Hence we get

$$
\begin{aligned}
\chi_{y}(x) \chi_{\eta}(D) u(t, x)= & \chi_{y}(x) \chi_{\eta}(D) I_{\psi(-t)}(x, D) \tilde{e}(-t, 0 ; x, D) I_{\varphi(t)}(x, D)^{-1} w(t, x) \\
& +\chi_{y}(x) \chi_{\eta}(D) I_{\psi(-t)}(x, D) \tilde{r}(-t ; x, D) I_{\varphi(t)}(x, D)^{-1} w(t, x) \\
= & U_{1}+U_{2} .
\end{aligned}
$$

Now, set $\tilde{e}(t, x, D)=I_{\varphi(t)}(x, D) \tilde{e}(-t, 0 ; x, D) I_{\varphi(t)}(x, D)^{-1}$, so that

$$
\begin{aligned}
U_{1} & =\chi_{y}(x) \chi_{\eta}(D) I_{\psi(-t)}(x, D) I_{\varphi(t)}(x, D)^{-1} \tilde{e}(t, x, D) w(t, x) \\
& =\chi_{y}(x) \chi_{\eta}(D) I_{\psi(-t)}(x, D) I_{\varphi(t)}(x, D)^{-1} \tilde{w}(t, x)
\end{aligned}
$$

where $\tilde{w}(t, x)=\tilde{e}(t, x, D) w(t, x) \in L^{2}$. Now by $(2.13)$

$$
\chi_{y}(x) \chi_{\eta}(D) I_{\psi(-t)}(x, D)=\tilde{\chi}_{\psi(-t)}(x, D),
$$


with $\operatorname{supp} \tilde{\chi}(x, \xi) \subset U_{\varepsilon} \times V_{\varepsilon}$. Using (2.13), we can see that the symbol of $\tilde{\chi}_{\psi(-t)}(x, D)$ belongs to $\gamma^{d} S\left(e^{-\rho t\langle\xi\rangle^{\kappa}}, g_{0}\right)$.

The symbol of $I_{\varphi(t)}^{-1}(x, D)$ belongs to $\gamma^{d} S\left(e^{C\langle x\rangle^{\sigma}\langle\xi\rangle^{\delta}}, g_{0}\right)$, and, since for any $\mu>0$ and $t>0$ there exists $C_{\mu}>0$ such that

$$
C\langle x\rangle^{\sigma}\langle\xi\rangle^{\delta} \leq \mu t\langle\xi\rangle^{\kappa}+C_{\mu} t^{-\delta / \sigma}\langle x\rangle^{\kappa},
$$

we have that the symbol of $I_{\varphi(t)}^{-1}(x, D)$ belongs to $\gamma^{d} S\left(e^{\mu t\langle\xi\rangle^{\kappa}+C_{\mu} t^{-\delta / \sigma}\langle x\rangle^{\kappa}}, g_{0}\right)$. We obtain that

$$
U_{1} \in H_{\rho t-\mu t,-C_{\mu} t^{-\delta / \sigma}}^{\kappa}
$$

Choosing $\mu$ small enough we obtain

$$
U_{1} \in H_{\rho / 2 t,-C t^{-\delta / \sigma}}^{\kappa},
$$

for some $C>0$. Concerning $U_{2}$, we have

$$
\begin{aligned}
\sigma\left(\chi_{y}(x) \chi_{\eta}(D) I_{\psi(-t)}(x, D)\right) & \in \gamma^{d} S\left(e^{-\rho t\langle\xi\rangle^{\kappa}}, g_{0}\right) \\
\tilde{r}(x, \xi) & \in \mathscr{C}^{1}\left([-T, T] ; \gamma^{d} S\left(e^{-\varepsilon_{0}(\langle x\rangle\langle\xi\rangle)^{1 /(d+s-1)}}, g_{0}\right)\right) \\
\sigma\left(I_{\varphi(t)}(x, D)^{-1}\right) & \in \gamma^{d} S\left(e^{C\langle x\rangle^{\sigma}\langle\xi\rangle^{\delta}}, g_{0}\right) .
\end{aligned}
$$

Since $\sigma>0$, then $\delta<1 / d$, and we can choose $s$ near 1 so that $\delta \leq 1 /(d+s-1)$, then

$$
\sigma\left(\tilde{r}(x, D) I_{\varphi(t)}(x, D)\right) \in \mathscr{C}^{1}\left([-T, T] ; \gamma^{d} S\left(1, g_{0}\right)\right)
$$

and we obtain $U_{2} \in H_{\rho t,-C}^{\kappa}$. This ends the proof of the Theorem.

\section{Appendix.}

Proof of Lemma 2.3. From the identity $\prod_{j=1}^{n}(1+t)^{\alpha_{j}}=(1+t)^{|\alpha|}$, one easily get $\sum_{\left|\alpha^{\prime}\right|=j}\left(\begin{array}{c}\alpha \\ \alpha^{\prime}\end{array}\right)=\left(\begin{array}{c}|\alpha| \\ j\end{array}\right)$, then

$$
\begin{aligned}
& \sum_{\substack{\alpha^{\prime}+\alpha^{\prime \prime}=\alpha \\
\left|\alpha^{\prime}\right| \geq k}}\left(\begin{array}{c}
\alpha \\
\alpha^{\prime}
\end{array}\right)\left(\left|\alpha^{\prime}\right|+r\right) !^{d}\left(\left|\alpha^{\prime \prime}\right|+s\right) !^{d} q^{-\left|\alpha^{\prime}\right|} \\
& =\sum_{j=k}^{|\alpha|} \sum_{\left|\alpha^{\prime}\right|=j}\left(\begin{array}{c}
\alpha \\
\alpha^{\prime}
\end{array}\right)\left(\left|\alpha^{\prime}\right|+r\right) !^{d}\left(|\alpha|-\left|\alpha^{\prime}\right|+s\right) !^{d} q^{-\left|\alpha^{\prime}\right|} \\
& =\sum_{j=k}^{|\alpha|}\left(\begin{array}{c}
|\alpha| \\
j
\end{array}\right)(j+r) !^{d}(|\alpha|-j+s) !^{d} q^{-j} .
\end{aligned}
$$
have

Since $(j+r) ! \leq 2^{j+r}(j+r-t) ! t !$ and $\left(\begin{array}{c}r+s-t \\ r-t\end{array}\right)\left(\begin{array}{c}|\alpha| \\ j\end{array}\right) \leq\left(\begin{array}{c}|\alpha|+r+s-t \\ j+r-t\end{array}\right)$ we 


$$
\begin{aligned}
\left(\begin{array}{c}
|\alpha| \\
j
\end{array}\right)(j+r) !^{d}(|\alpha|-j+s) !^{d} & \leq\left(\begin{array}{c}
|\alpha| \\
j
\end{array}\right) 2^{d(j+r)}(j+r-t) !^{d} t !^{d}(|\alpha|-j+s) !^{d} \\
& =2^{d(j+r)} t !^{d} \frac{\left(\begin{array}{c}
|\alpha| \\
j
\end{array}\right)}{\left(\begin{array}{c}
|\alpha|+r+s-t \\
j+r-t
\end{array}\right)}(|\alpha|+r+s-t) !^{d} \\
& \leq \frac{2^{d(j+r)} t !^{d}}{\left(\begin{array}{c}
r+s-t \\
r-t
\end{array}\right)^{d}}(|\alpha|+r+s-t) !^{d}
\end{aligned}
$$

Then

$$
\sum_{\alpha^{\prime}+\alpha^{\prime \prime}=\alpha}\left(\begin{array}{c}
\alpha \\
\alpha^{\prime}
\end{array}\right)\left(\left|\alpha^{\prime}\right|+r\right) !^{d}\left(\left|\alpha^{\prime \prime}\right|+s\right) !^{d} q^{-\left|\alpha^{\prime}\right|} \leq \frac{2^{d r} t^{d}}{\left(\begin{array}{c}
r+s-t \\
r-t
\end{array}\right)}(|\alpha|+r+s-t) !^{d} 2^{d j} q^{-j} .
$$

Since $\sum_{j=k}^{|\alpha|} 2^{d j} q^{-j} \leq\left(2^{d} / q\right)^{k} /\left(1-\left(2^{d} / q\right)\right)$, we obtain the proof.

Proof of Lemma 2.8. Let

$$
\begin{gathered}
X_{j}=\sum_{j=1}^{n} \frac{\partial \varphi_{i}}{\partial x_{j}} \frac{\partial}{\partial z_{i}}+\sum_{j=1}^{n} \frac{\partial \psi_{i}}{\partial x_{j}} \frac{\partial}{\partial \zeta_{i}}+\frac{\partial}{\partial x_{j}} \\
Y_{j}=\sum_{j=1}^{n} \frac{\partial \varphi_{i}}{\partial \xi_{j}} \frac{\partial}{\partial z_{i}}+\sum_{j=1}^{n} \frac{\partial \psi_{i}}{\partial \xi_{j}} \frac{\partial}{\partial \zeta_{i}}+\frac{\partial}{\partial \xi_{j}},
\end{gathered}
$$

for $j=1, \ldots, n$. For $g(x, \xi, z, \zeta) \in \mathscr{C}^{\infty}\left(\boldsymbol{R}^{n} \times \boldsymbol{R}^{n} \backslash\{0\} \times \boldsymbol{R}^{n} \times \boldsymbol{R}^{n} \backslash\{0\}\right)$ we have

$$
\begin{gathered}
\frac{\partial}{\partial x_{j}}[g(x, \xi, \varphi(x, \xi), \psi(x, \xi))]=\left.X_{j} g(x, \xi, z, \zeta)\right|_{\substack{z=\varphi(x, \xi) \\
\zeta=\psi(x, \xi)}} \\
\frac{\partial}{\partial \xi_{j}}[g(x, \xi, \varphi(x, \xi), \psi(x, \xi))]=\left.Y_{j} g(x, \xi, z, \zeta)\right|_{\begin{array}{c}
z=\varphi(x, \xi) \\
\zeta=\psi(x, \xi)
\end{array}},
\end{gathered}
$$

therefore

$$
\partial_{\xi}^{\alpha} \partial_{x}^{\beta} f(\varphi(x, \xi), \psi(x, \xi))=\left.X^{\beta} Y^{\alpha} f(x, \xi)\right|_{\substack{z=\varphi(x, \xi) \\ \zeta=\psi(x, \xi)}}
$$

Now let us prove that

$$
\begin{aligned}
\left|\partial_{\xi}^{\alpha} \partial_{x}^{\beta} \partial_{\zeta}^{\lambda} \partial_{z}^{\mu} Y^{\tilde{\alpha}} X^{\tilde{\beta}} f(z, \zeta)\right| \leq & C|\alpha+\alpha+\lambda+\beta+\tilde{\beta}+\mu| !^{d} \\
& \times \rho^{-|\alpha+\alpha+\beta+\tilde{\beta}+\lambda+\mu|}\left[1+\frac{m_{\varphi}(x, \xi)}{\langle\varphi(x, \xi)\rangle}+\frac{m_{\psi}(x, \xi)}{|\psi(x, \xi)|}\right]^{|\tilde{\alpha}+\tilde{\beta}|} \\
& \times m(x, \xi) \eta_{1}(x, \xi)^{-|\alpha+\tilde{\alpha}|} \eta_{2}(x, \xi)^{-|\beta+\tilde{\beta}|}|\zeta|^{-|\lambda|}\langle z\rangle^{-|\mu|}
\end{aligned}
$$

for some $C$ and $\rho$. 
We proceed by induction on $|\tilde{\alpha}+\tilde{\beta}|$. If $|\tilde{\alpha}+\tilde{\beta}|=0$, inequality (6.8) holds true by hypothesis for any $\rho<\rho_{0}$. Assume (6.8) for $|\tilde{\alpha}+\tilde{\beta}| \leq \ell-1<k$. We have

$$
\begin{aligned}
\partial_{\xi}^{\alpha} \partial_{x}^{\beta} \partial_{\zeta}^{\lambda} \partial_{z}^{\mu} Y_{j} Y^{\tilde{\alpha}} X^{\tilde{\beta}} f(z, \zeta) & =\partial_{\xi}^{\alpha} \partial_{x}^{\beta} Y_{j} \partial_{\zeta}^{\lambda} \partial_{z}^{\mu} Y^{\tilde{\alpha}} X^{\tilde{\beta}} f(z, \zeta) \\
& =\partial_{\xi}^{\alpha} \partial_{x}^{\beta}\left[\sum_{i=1}^{n} \frac{\partial \varphi_{i}}{\partial \xi_{j}} \frac{\partial}{\partial z_{i}}+\sum_{i=1}^{n} \frac{\partial \psi_{i}}{\partial \xi_{j}} \frac{\partial}{\partial \zeta_{i}}+\frac{\partial}{\partial \xi_{j}}\right] \partial_{\zeta}^{\lambda} \partial_{z}^{\mu} Y^{\tilde{\alpha}} X^{\tilde{\beta}} f(z, \zeta)
\end{aligned}
$$

Let $I_{i}=\partial_{\xi}^{\alpha} \partial_{x}^{\beta}\left(\partial \varphi_{i} / \partial \xi_{j}\right)\left(\partial / \partial z_{i}\right) \partial_{\zeta}^{\lambda} \partial_{z}^{\mu} Y^{\tilde{\alpha}} X^{\tilde{\beta}} f(z, \zeta)$, we have

$$
\begin{aligned}
\left|I_{i}\right| \leq & \sum_{\substack{\alpha^{\prime}+\alpha^{\prime \prime}=\alpha \\
\beta^{\prime}+\beta^{\prime \prime}=\beta}}\left(\begin{array}{c}
\alpha \\
\alpha^{\prime}
\end{array}\right)\left(\begin{array}{c}
\beta \\
\beta^{\prime}
\end{array}\right)\left|\varphi_{i\left(\beta^{\prime}\right)}^{\left(\alpha^{\prime}+e_{j}\right)}\right|\left|\partial_{\xi}^{\alpha^{\prime \prime}} \partial_{x}^{\beta^{\prime \prime}} \partial_{\zeta}^{\lambda} \partial_{z}^{\mu+e_{i}} Y^{\tilde{\alpha}} X^{\tilde{\beta}} f(z, \zeta)\right| \\
\leq & C \sum_{\substack{\alpha^{\prime}+\alpha^{\prime \prime}=\alpha \\
\beta^{\prime}+\beta^{\prime \prime}=\beta}}\left(\begin{array}{c}
\alpha \\
\alpha^{\prime}
\end{array}\right)\left(\begin{array}{c}
\beta \\
\beta^{\prime}
\end{array}\right)\left(\left|\alpha^{\prime}\right|+1\right) !^{d}\left|\beta^{\prime}\right| !^{d} \rho_{0}^{-\left|\alpha^{\prime}+\beta^{\prime}\right|-1} m_{\varphi}(x, \xi) \eta_{1}(x, \xi)^{-\left|\alpha^{\prime}\right|-1} \eta_{2}(x, \xi)^{-\left|\beta^{\prime}\right|} \\
& \times\left(\left|\alpha^{\prime \prime}+\tilde{\alpha}+\lambda+\beta^{\prime \prime}+\tilde{\beta}+\mu\right|+1\right) !^{d} \rho^{-\left|\alpha^{\prime \prime}+\tilde{\alpha}+\beta^{\prime \prime}+\tilde{\beta}+\lambda+\mu\right|-1}\left[1+\frac{m_{\varphi}(x, \xi)}{\langle\varphi(x, \xi)\rangle}+\frac{m_{\psi}(x, \xi)}{|\psi(x, \xi)|}\right]^{|\tilde{\alpha}+\tilde{\beta}|} \\
& \times m^{\tilde{\beta}}(x, \xi) \eta_{1}(x, \xi)^{-\left|\alpha^{\prime \prime}+\tilde{\alpha}\right|} \eta_{2}(x, \xi)^{-\left|\beta^{\prime \prime}+\tilde{\beta}\right|}|\zeta|^{-|\lambda|}\langle z\rangle^{-|\mu|-1} \\
\leq & C \sum_{\alpha^{\prime}+\alpha^{\prime \prime}=\alpha}\left(\begin{array}{c}
\alpha \\
\alpha^{\prime}
\end{array}\right)\left|\alpha^{\prime}\right| !^{d}\left(\frac{\rho_{0}}{2 \rho}\right)^{-\left|\alpha^{\prime}\right|} \\
& \times \sum_{\beta^{\prime}+\beta^{\prime \prime}=\beta}\left(\begin{array}{c}
\beta \\
\beta^{\prime}
\end{array}\right)\left|\beta^{\prime}\right| !^{d}\left(\left|\alpha^{\prime \prime}+\tilde{\alpha}+\lambda+\beta^{\prime \prime}+\tilde{\beta}+\mu\right|+1\right) !^{d}\left(\frac{\rho_{0}}{\rho}\right)^{-\left|\beta^{\prime}\right|} \\
& \times \rho_{0}^{-1} \rho^{-|\alpha+\tilde{\alpha}+\beta+\tilde{\beta}+\lambda+\mu|-1}\left[1+\frac{m_{\varphi}(x, \xi)}{\langle\varphi(x, \xi)\rangle}+\frac{m_{\psi}(x, \xi)}{|\psi(x, \xi)|}\right]^{|\tilde{\alpha}+\tilde{\beta}|} \frac{m_{\varphi}(x, \xi)}{\langle z\rangle} \\
& \times m(x, \xi) \eta_{1}(x, \xi)^{-|\alpha+\tilde{\alpha}|-1} \eta_{2}(x, \xi)^{-|\beta+\tilde{\beta}|}|\zeta|^{-|\lambda|}\langle z\rangle^{-|\mu|} .
\end{aligned}
$$

Using Lemma 2.2 for the first sum and Lemma 2.3 for the second we have

$$
\begin{aligned}
\left|I_{i}\right| \leq & \frac{2 \rho C}{\left(\rho_{0}-\rho\right)\left(\rho_{0}-2 \rho\right)}(|\alpha+\beta+\tilde{\alpha}+\tilde{\beta}+\lambda+\mu|+1) !^{d} \\
& \times \rho^{-|\alpha+\tilde{\alpha}+\beta+\tilde{\beta}+\lambda+\mu|-1}\left[1+\frac{m_{\varphi}(x, \xi)}{\langle\varphi(x, \xi)\rangle}+\frac{m_{\psi}(x, \xi)}{|\psi(x, \xi)|}\right]^{|\tilde{\alpha}+\tilde{\beta}|} \frac{m_{\varphi}(x, \xi)}{\langle z\rangle} \\
& \times m(x, \xi) \eta_{1}(x, \xi)^{-|\alpha+\tilde{\alpha}|-1} \eta_{2}(x, \xi)^{-|\beta+\tilde{\beta}|}|\zeta|^{-|\lambda|}\langle z\rangle^{-|\mu|} .
\end{aligned}
$$

Similarly, if $J_{i}=\partial_{\xi}^{\alpha} \partial_{x}^{\beta}\left(\partial \psi_{i} / \partial \xi_{j}\right)\left(\partial / \partial \zeta_{i}\right) \partial_{\zeta}^{\lambda} \partial_{z}^{\mu} Y^{\tilde{\alpha}} X^{\tilde{\beta}} f(z, \zeta)$, we have 


$$
\begin{aligned}
\left|J_{i}\right| \leq & \frac{2 \rho C}{\left(\rho_{0}-\rho\right)\left(\rho_{0}-2 \rho\right)}(|\alpha+\tilde{\alpha}+\beta+\tilde{\beta}+\lambda+\mu|+1) !^{d} \\
& \times \rho^{-|\alpha+\tilde{\alpha}+\beta+\tilde{\beta}+\lambda+\mu|-1}\left[1+\frac{m_{\varphi}(x, \xi)}{\langle\varphi(x, \xi)\rangle}+\frac{m_{\psi}(x, \xi)}{|\psi(x, \xi)|}\right]^{|\tilde{\alpha}+\tilde{\beta}|} \frac{m_{\psi}(x, \xi)}{|\zeta|} \\
& \times m(x, \xi) \eta_{1}(x, \xi)^{-|\alpha+\tilde{\alpha}|-1} \eta_{2}(x, \xi)^{-|\beta+\tilde{\beta}|}|\zeta|^{-|\lambda|}\langle z\rangle^{-|\mu|}
\end{aligned}
$$

For $K_{i}=\partial_{\xi}^{\alpha} \partial_{x}^{\beta} \frac{\partial}{\partial \xi_{j}} \partial_{\zeta}^{\lambda} \partial_{z}^{\mu} Y^{\tilde{\alpha}} X^{\tilde{\beta}} f(z, \zeta)$, we have

$$
\begin{aligned}
\left|K_{i}\right| \leq & (|\alpha+\tilde{\alpha}+\beta+\tilde{\beta}+\lambda+\mu|+1) ! \rho^{-|\alpha+\tilde{\alpha}+\beta+\tilde{\beta}+\lambda+\mu|-1}\left[1+\frac{m_{\varphi}(x, \xi)}{\langle\varphi(x, \xi)\rangle}+\frac{m_{\psi}(x, \xi)}{|\psi(x, \xi)|}\right]^{|\tilde{\alpha}+\tilde{\beta}|} \\
& \times m(x, \xi) \eta_{1}(x, \xi)^{-|\alpha+\tilde{\alpha}|-1} \eta_{2}(x, \xi)^{-|\beta+\tilde{\beta}|}|\zeta|^{-|\lambda|}\langle z\rangle^{-|\mu|}
\end{aligned}
$$

Putting together estimations for $\left|I_{i}\right|,\left|J_{i}\right|$ and $\left|K_{i}\right|$, we obtain (6.8) for $\left(\tilde{\alpha}+e_{j}, \tilde{\beta}\right)$ if we choose $\rho>0$ and $C>0$ suitably. Similarly we can prove (6.8) for $\left(\tilde{\alpha}, \tilde{\beta}+e_{j}\right)$. Using (6.8) with $\alpha=\tilde{\beta}=\lambda=\mu=0$, and taking into account (6.7), we obtain (2.3).

LEMMA 6.1. Let $M=\left(\begin{array}{cc}M_{11} & M_{12} \\ M_{21} & I_{q}\end{array}\right)$ be a $2 q \times 2 q$ matrix and let $\bar{M}=M_{11}-$ $M_{12} M_{21}$. We have

(1) $\operatorname{det} M=\operatorname{det} \bar{M}$;

(2) $\quad M^{-1}=\left(\begin{array}{cc}\bar{M}^{-1} & -\bar{M}^{-1} M_{12} \\ -M_{21} \bar{M}^{-1} & I_{q}+M_{21} \bar{M}^{-1} M_{12}\end{array}\right)$.

Proof. The proof follows from the identities

$$
\begin{aligned}
& M=\left(\begin{array}{cc}
I_{q} & M_{12} \\
0 & I_{q}
\end{array}\right)\left(\begin{array}{cc}
M_{11}-M_{12} M_{21} & 0 \\
M_{21} & I
\end{array}\right), \\
& M\left(\begin{array}{cc}
\bar{M}^{-1} & -\bar{M}^{-1} M_{12} \\
-M_{21} \bar{M}^{-1} & I_{q}+M_{21} \bar{M}^{-1} M_{12}
\end{array}\right)=\left(\begin{array}{cc}
I_{q} & 0 \\
0 & I_{q}
\end{array}\right) .
\end{aligned}
$$

LEMMA 6.2. Let

$$
M=\left(\begin{array}{ccccc}
\alpha_{1} \beta_{1} & -\alpha_{2} & & \cdots & -\alpha_{n} \\
\beta_{2} & 1 & 0 & \ldots & 0 \\
& 0 & \ddots & & \vdots \\
\vdots & & & \ddots & 0 \\
\beta_{n} & 0 & \ldots & 0 & 1
\end{array}\right)
$$

then

(1) $\operatorname{det} M=\alpha \beta=\sum_{j=1}^{n} \alpha_{j} \beta_{j} ;$ 
(2)

$$
M^{-1}=\frac{1}{\alpha \beta}\left(\begin{array}{ccccc}
1 & \alpha_{2} & & \ldots & \alpha_{n} \\
-\beta_{2} & \alpha \beta-\alpha_{2} \beta_{2} & -\alpha_{3} \beta_{2} & \ldots & -\alpha_{n} \beta_{2} \\
& -\alpha_{2} \beta_{3} & \ddots & & \vdots \\
\vdots & \vdots & & \ddots & -\alpha_{n} \beta_{n-1} \\
-\beta_{n} & -\alpha_{2} \beta_{n} & \ldots & -\alpha_{n-1} \beta_{n} & \alpha \beta-\alpha_{n} \beta_{n}
\end{array}\right) .
$$

Proof. The proof follows easily from Lemma 6.1.

\section{References}

[1] W. Craig, Les moments microlocaux et la régularité des solutions de l'équation de Schrödinger, Sémin. Équ. Dériv. Partielles, 1995-1996, École Polytech., Palaiseau, 1996, XX.1-XX.24.

[2] W. Craig, Microlocal moments and regularity of solutions of Schrödinger's equation, Math. Phys. Electron. J., 3 (1997), Paper 2, 22 pp. (electronic).

[ 3 ] W. Craig, T. Kappeler and W. Strauss, Gain of regularity for equations of $K d V$ type, Ann. Inst. H. Poincaré Anal. Non Linéaire, 9 (1992), no. 2, 147-186.

[ 4] W. Craig, T. Kappeler and W. Strauss, Microlocal dispersive smoothing for the Schrödinger equation, Comm. Pure Appl. Math., 48 (1995), no. 8, 769-860.

[5] A. De Bouard, N. Hayashi and K. Kato, Regularizing effect for the (generalized) Korteweg-de Vrie equations and nonlinear Schrödinger equations, Ann. Inst. Henri Poincaré Analyse Non Linear, vol. 12 (1995), pp. 673-725.

[6] S. Doi, On the Cauchy problem for Schrödinger type equations and the regularity of solutions, J. Math. Kyoto Univ., 34 (1994), no. 2, 319-328.

[ 7 ] S. Doi, Remarks on the Cauchy problem for Schrödinger-type equations, Comm. Partial Differential Equations, 21 (1996), no. 1-2, 163-178.

[ 8 ] S. Doi, Smoothing effects of Schrödinger evolution groups on Riemannian manifolds, Duke Math. J., 82 (1996), no. 3, 679-706.

[9] S. Doi, Smoothing properties of Schrödinger equations and the behavior of geodesic flows, Kōkyūroku, no. 937, (1996), 7-16, Vanishing cycles of $D$-modules and their applications (Japanese) (Kyoto, 1994).

[10] S. Doi, Regularizing effects for Schrödinger type equations, Sūgaku, 49 (1997), no. 1, 38-47.

[11] S. Doi, Smoothing effect for Schrödinger evolution equation via commutator algebra, Sémin. Équ. Dériv. Partielles, 1996-1997, École Polytech., Palaiseau, 1997, XX.1-XX.15.

[12] Y. Hamada, J. Leray and C. Wagschal, Systèmes d'équations aux dérivées partielles à caractéristiques multiples: problème de Cauchy ramifié; hyperbolicité partielle, J. Math. Pures Appl. (9), 55 (1976), no. 3, 297-352.

[13] N. Hayashi and S. Saitoh, Analyticity and smoothing effect for the Schrödinger equation, Ann. Inst. H. Poincaré Phys. Théor., 52 (1990), no. 2, 163-173.

[14] N. Hayashi, K. Nakamitsu and M. Tsutsumi, On solutions of the initial value problem for the nonlinear Schrödinger equations in one space dimension, Math. Z., 192 (1986), no. 4, 637-650.

[15] N. Hayashi, K. Nakamitsu and M. Tsutsumi, On solutions of the initial value problem for the nonlinear Schrödinger equations, J. Funct. Anal., 71 (1987), no. 2, 218-245.

[16] A. Jensen, Commutator methods and a smoothing property of the Schrödinger evolution group, Math. Z., 191 (1986), no. 1, 53-59.

[17] A. Jensen and P. Perry, Commutator methods and Besov space estimates for Schrödinger operators, J. Operator Theory, 14 (1985), no. 1, 181-188.

[18] K. Kajitani, The Cauchy problem for Schrödinger type equations with variable coefficients, J. Math. Soc. Japan, 50 (1998), no. 1, 179-202.

[19] K. Kajitani, Analytically smoothing effect for Schrödinger equations, Proceedings of the International Conference on Dynamical Systems \& Differential Equations, (eds. W. Chen and S. Hu), Southwest Missouri State University, 1996, An added Volume I to Discrete Contin. Dynam. Systems, 1998, pp. $350-353$. 
[20] K. Kajitani, Smoothing effect in Gevrey classes for Schrödinger equations I, Lecture Notes in Pure and Appl. Math., 233 (2003), 269-285.

[21] K. Kajitani, Smoothing effect in Gevrey classes for Schrödinger equations II, Ann. Univ. Ferrara-Sez. VII-Sc. Mat. Suppl., Vol. XLV (1999), 173-186.

[22] K. Kajitani and A. Baba, The Cauchy problem for Schrödinger type equations, Bull. Sci. Math., 119 (1995), no. 5, 459-473.

[23] K. Kajitani and T. Nishitani, The hyperbolic Cauchy problem, Lecture Notes in Math., 1505, Springer-Verlag, Berlin, 1991.

[24] K. Kajitani and S. Wakabayashi, Analytically smoothing effect for Schrödinger type equations with variable coefficients, Proceeding of Symposium of P.D.E., University of Delaware, 1997.

[25] K. Kato and K. Taniguchi, Gevrey regularizing effect for nonlinear Schrödinger equations, Osaka J. Math., vol. 33 (1996), pp. 863-880.

[26] Y. Morimoto, L. Robbiano and C. Zuily, Remark on the analytic smoothing effect for the Schrödinger equation, Indiana Univ. Math. J., 49 (2000), no. 4, 1563-1579.

[27] L. Robbiano and C. Zuily, Microlocal analytic smoothing effect for the Schrödinger equation, Duke Math. J., 100 (1999), no. 1, 93-129.

[28] L. Robbiano and C. Zuily, Effet régularisant microlocal analytique pour l'équation de Schrödinger, Sémin. Équ. Dériv. Partielles, 1997-1998, École Polytech., Palaiseau, 1998, XIX.1-XIX.14.

[29] L. Robbiano and C. Zuily, Effet régularisant microlocal analytique pour l'équation de Schrödinger: le cas des données oscillantes, Comm. Partial Differential Equations, 25 (2000), no. 9-10, 1891-1906.

[30] J. T. Schwartz, Nonlinear Functional Analysis, Note on Mathematics and its Applications, 1969, Gordon \& Breach.

\author{
Kunihiko KaJITANI \\ Institute of Mathematics \\ University of Tsukuba \\ 1-1-1 Tennōdai, 305 \\ Tsukuba, Ibaraki \\ Japan \\ E-mail: kajitani@math.tsukuba.ac.jp
}

\author{
Giovanni TAgLialatela \\ Institute of Mathematics \\ University of Tsukuba \\ 1-1-1 Tennōdai, 305 \\ Tsukuba, Ibaraki \\ Japan \\ E-mail: taglia@math.tsukuba.ac.jp
}

